\title{
A CRISPR interference platform for selective downregulation of gene expression in Borrelia burgdorferi.
}

Constantin N. Takacsa,b,c,d, Molly Scottb,c, Yunjie Chang ${ }^{b, e}$, Zachary A. Kloos $^{b, f}$, Irnov Irnova,b,c,d, Patricia A. Rosag, Jun Liub,e, and Christine Jacobs-Wagnera,b,c,d,e,\#

a Department of Biology and ChEM-H Institute, Stanford University, Stanford, CA, 94305, USA.

${ }^{\mathrm{b}}$ Microbial Sciences Institute, Yale West Campus, West Haven, CT, 06516, USA.

c Department of Molecular, Cellular, and Developmental Biology, Yale University, New Haven, CT, 06511, USA.

d Howard Hughes Medical Institute, Stanford University, CA, 94305, USA.

e Department of Microbial Pathogenesis, Yale University School of Medicine, New Haven, CT, 06511, USA.

f Microbiology Program, Yale University, New Haven, CT, 06511, USA.

g Laboratory of Bacteriology, Rocky Mountain Laboratories, Division of Intramural Research, National Institute of Allergy and Infectious Diseases, National Institutes of Health, Hamilton, MT, 59840, USA.

Running Head: CRISPR interference in Borrelia burgdorferi.

\# To whom correspondence should be addressed. Email: jacobs-wagner@stanford.edu.

Keywords: CRISPR, Borrelia, Lyme disease, spirochete, dCas9, cell morphogenesis, bacteria, MreB, RodA, Ftsl, 


\section{ABSTRACT}

The spirochete Borrelia burgdorferi causes Lyme disease, an increasingly prevalent infection. While previous studies have provided important insight into B. burgdorferi biology, many aspects, including basic cellular processes, remain underexplored. To help speed up the discovery process, we adapted a CRISPR interference (CRISPRi) platform for use in $B$. burgdorferi. For efficiency and flexibility of use, we generated various CRISPRi template constructs that produce different basal and induced levels of dcas 9 and carry different antibiotic resistance markers. We characterized the effectiveness of our CRISPRi platform by targeting the motility and cell morphogenesis genes $f l a B, \operatorname{mre} B$, $\operatorname{rod} A$, and $f t s l$, whose native expression levels span two orders of magnitude. For all four genes, we obtained gene repression efficiencies of at least $95 \%$. We showed by darkfield microscopy and cryo-electron tomography that flagellin (FlaB) depletion reduced the length and number of periplasmic flagella, which impaired cellular motility and resulted in cell straightening. Depletion of Ftsl caused cell filamentation, implicating this protein in cell division in B. burgdorferi. Finally, localized cell bulging in MreB- and RodA-depleted cells matched the locations of new peptidoglycan insertion specific to spirochetes of the Borrelia genus. These results therefore implicate MreB and RodA in the particular mode of cell wall elongation of these bacteria. Collectively, our results demonstrate the efficiency and ease of use of our B. burgdorferi CRISPRi platform, which should facilitate future genetic studies of this important pathogen. 


\section{IMPORTANCE}

Gene function studies are facilitated by the availability of rapid and easy-to-use genetic tools. Homologous recombination-based methods traditionally used to genetically investigate gene function remain cumbersome to perform in B. burgdorferi, as they often are relatively inefficient. In comparison, our CRISPRi platform offers an easy and fast method to implement as it only requires a single plasmid transformation step and IPTG addition to obtain potent (>95\%) downregulation of gene expression. To facilitate studies of various genes in wild-type and genetically modified strains, we provide over 30 CRISPRi plasmids that produce distinct levels of dcas9 expression and carry different antibiotic resistance markers. Our CRISPRi platform represents a useful and efficient complement to traditional genetic and chemical methods to study gene function in $B$. burgdorferi. 


\section{INTRODUCTION}

Borrelia burgdorferi, a spirochetal bacterium, is maintained in nature via a transmission cycle between a tick vector and a warm-blooded host, such as a wild mouse (1). A bite by a $B$. burgdorferi-colonized tick can lead to transmission of the bacterium to humans. In the absence of timely antibiotic treatment, infection by $B$. burgdorferi causes Lyme disease, a prevalent vector-borne disease in temperate regions of the Northern hemisphere (2). The infection can result in a wide range of symptoms, from malaise, fever, and a characteristic skin rash during early stages of the disease, to cardiac, neurologic, or articular pathologies in later stages (3). The rapid rise in Lyme disease incidence in recent years (2) underscores the need for a detailed understanding of not only the disease, but also the pathogen itself.

Studying the biology of a pathogen is greatly facilitated by the genetic tractability of the organism. For this reason, a diverse set of genetic reagents and techniques have been developed and validated for use in B. burgdorferi (4-6). However, creating gene deletion mutants in B. burgdorferi remains tedious and time-consuming. Homologous recombination of suicide vectors is needed to create gene deletion mutants. In B. burgdorferi, this process occurs at frequencies of $\sim 10^{-7}(7-9)$, which are close to the $10^{-9}$ to $10^{-7}$ range of frequencies at which spontaneous mutants conferring resistance to common selection antibiotics arise (10). This inefficient process is compounded by $B$. burgdorferi's slow in vitro growth rate, with typical doubling times in the range of 5 to 18 hours (11-16). Genetic modification is particularly challenging for genes essential for viability; such genes cannot be deleted, and generation of conditional mutants (17) often requires multiple transformation steps and is therefore even slower and less efficient.

The development of CRISPR (clustered regularly interspaced palindromic repeats) interference $(18,19)$, or CRISPRi, provides an attractive complement to traditional genetic manipulation protocols based on homologous recombination. CRISPRi is a gene product 
depletion method that requires two components. One of them is dCas9, a catalytically inactive version of Cas9, which is the nuclease component of a bacterial adaptive immunity system against invading DNA molecules $(18,20,21)$. The other component is a short guide RNA molecule, or sgRNA. The sgRNA (Fig. 1A) contains a base-pairing region and a dCas9 recognition loop, which is called a dCas9 handle $(18,20)$. The base-paring region is usually a 20-nucleotide stretch complementary to the target DNA strand $(18,19)$. Proper targeting also requires a protospacer adjacent motif, or PAM, located in the DNA next to the base-pairing region. When co-expressed, dCas9 and the sgRNA form a complex that scans the DNA until it finds a PAM-proximal sequence complementary to the sgRNA's base-pairing region (22). There, the dCas9-sgRNA complex stably binds to the DNA. When dCas9 is targeted to a promoter or operator, it blocks transcription initiation. When dCas9 is targeted to the coding sequence or the $5^{\prime}$ untranslated region (5' UTR) of a gene, it blocks transcription elongation (18). Together, the required sgRNA-DNA complementary base pairing and presence of an adjacent PAM site render CRISPRi highly specific (18). Since its development, the CRISPRi method has been adapted for the study of a wide variety of organisms and cell types, from mammalian cells and yeast (23) to various bacterial species, including E. coli (18), Bacillus subtilis $(24,25)$, Caulobacter crescentus (26, 27), Mycobacterium tuberculosis (28-30), and Leptospira interrogans (31) to name just a few.

Here, we have adapted the CRISPRi system for use in B. burgdorferi. We present multiple versions of the platform that are characterized by efficient gene product downregulation, ease of use, and relatively fast clone generation. We have evaluated the functionality of these versions by targeting genes that have broadly varied native expression levels and are involved in B. burgdorferi motility, cell shape determination, and cell division. 


\section{RESULTS}

\section{Constructs and strains for sgRNA and dcas9 expression in B. burgdorferi.}

To adapt the CRISPRi system for use in B. burgdorferi, we assembled expression cassettes for sgRNAs and dcas9 (Fig. 1A-B). The mature sgRNA cassettes that we designed contain the sgRNA sequence fused to the transcriptional start site of a constitutive promoter (Fig. 1A). To generate mature sgRNA cassettes, we first assembled "template" cassettes, which contain the promoter driving sgRNA expression, a 500 base pair DNA filler sequence derived from a firefly luciferase gene (32) and the sgRNA's dCas9 handle (Fig. S1A). We then released the DNA filler sequence by digestion with Sapl (or its isoschiomers BspQI or LguRI) and ligated in its place a pair of annealed primers (Fig. S1A-B). We designed these primers such that they encode the sgRNA's base-pairing region (see Materials and Methods). For sgRNA expression, we primarily employed a synthetic promoter, J23119, hereafter referred to as $\mathrm{P}_{\text {syn, }}$ which was previously used to drive sgRNA expression in $E$. coli $(18,19)$. Using a transcriptional fusion to mCherry, we showed that $\mathrm{P}_{\text {syn }}$ is active in B. burgdorferi (Fig. S1C-D). To drive sgRNA expression, we also tested multiple $B$. burgdorferi promoters (Table S1) whose strengths we previously characterized (Fig. S1D) (6). The nature of the promoter driving sgRNA expression, however, did not appear to affect the functionality of the CRISPRi platform (see below). We generated both the template and the mature sgRNA cassettes in B. burgdorferi/E. coli shuttle vectors and refer to them as sgRNA shuttle vectors (Fig. 1C and S1A and Table 1).

To express dcas9, we generated a dcas9/lacl cassette (Fig. 1B), which contains the dcas9 gene controlled by the IPTG-inducible $\mathrm{P}_{P Q E 30}$ promoter and a constitutively expressed lacl gene, both derived from plasmid pJSB252 (32). When the dcas9/lacl cassette is expressed from a $B$. burgdorferi shuttle vector, we hereafter refer to such a vector as a dcas 9 shuttle vector (Fig. 1D and Table 1). To facilitate cloning of a sgRNA cassette into the dcas 9 shuttle vector, we generated cloning intermediates that contain a rifampin resistance cassette placeholder (Fig. 
1E and S1E and Table 1). Replacement of the rifampin cassette with sgRNA cassettes yielded all-in-one CRISPRi shuttle vectors (Fig. 1F and S1E and Table 1). To facilitate use of the CRISPRi platform in a variety of $B$. burgdorferi strains, including ones that already contain antibiotic resistance markers from prior genetic modifications, we generated five variations of the dcas9 shuttle vectors, each carrying a different antibiotic resistance marker (Table 1).

We also inserted the dcas $9 / / \mathrm{lac} /$ cassette into an intergenic region of the chromosome of the infectious, transformable, B31-derived B. burgdorferi strain B31-A3-68 $\triangle$ bbe02::PflaB-aadA (33), generating strain CJW_Bb362 (Fig. 1G and Table 2). This strain allows for stable maintenance of the dcas9/lac/ cassette in the absence of antibiotic selection. It requires transformation with a sgRNA shuttle vector (Fig. 1C) to generate a CRISPRi strain for protein depletion, while transformation with an empty shuttle vector yields a control strain.

Regardless of the CRISPRi version used (all-in-one CRISPRi shuttle vector or strain CJW_Bb362 transformed with an sgRNA shuttle vector), in the absence of IPTG (uninduced condition), cells of $B$. burgdorferi CRISPRi strains had their dcas 9 expression repressed by the binding of Lacl to the lacO sites within the $\mathrm{P}_{p Q E 30}$ promoter (Fig. 1H). IPTG addition to the cultures (induced condition) releases $\mathrm{Lacl}$ from $\mathrm{P}_{p Q E 30}$, derepresses transcription from this promoter, and leads to synthesis of dCas9. Nonetheless, some background dcas 9 expression occurred in the absence of IPTG. As discussed below, this proved problematic when targeting certain B. burgdorferigenes. To decrease this basal dcas 9 expression, we mutated either the -10 promoter region of $\mathrm{P}_{P Q E 30}$ or the ribosome-binding site upstream of the dcas 9 translational start codon in the background of the dcas9 or the all-in-one CRISPRi shuttle vectors (Fig. $1 \mathrm{H}$ and S1F and Table 1). One of the promoter mutations (-10TC), as well as the ribosome-binding site mutation (RBSmut) proved effective in reducing background expression of $d \operatorname{cas} 9$ and were analyzed in greater detail (see below). 
Altogether, we generated and characterized four versions of the $B$. burgdorferi CRISPRi system. One is based on chromosomal expression of dcas 9 paired with plasmid-based expression of the sgRNA. The others are all-in-one plasmid-based versions that express both dcas 9 and the sgRNA from the same plasmid. The plasmid-based versions differ in whether the promoter that controls dcas 9 expression carries no mutation, a promoter mutation (-10TC) or the ribosomal binding site mutation (RBSmut).

\section{Inducible expression of dcas9 in B. burgdorferi.}

To characterize dcas9 expression in each of the four versions of our $B$. burgdorferi CRISPRi platform, we generated four corresponding sgRNA-free control strains (Table 2). We first measured, by quantitative real-time polymerase-chain reaction (qRT-PCR), their basal dcas 9 expression in the absence of IPTG induction. As expected, all control strains (CJW_Bb242, CJW_Bb410, and CJW_Bb411) harboring dcas9 on a shuttle vector had higher levels of basal dcas9 expression than the control strain (CJW_Bb430) carrying a chromosomal dcas9 (Fig. 2A). Expression of lacl was also about five-fold higher in these strains than in the chromosomal dcas9 strain (Fig. 2B). These levels could reflect copy-number differences between the shuttle vector and the chromosome, previously reported to be in a ratio of about 5:1 (34-36). They could also reflect changes in DNA topology and genomic context that are known to affect gene expression in B. burgdorferi (36-38).

Basal levels of dcas 9 expression varied among the control strains carrying the $d$ cas $9 / / a c l$ cassette on a shuttle vector, with the highest level found in strain CJW_Bb242 (Fig. 2A), which has dcas9 expression controlled by the parental $\mathrm{P}_{p Q E 30}$ promoter (Fig. $\left.1 \mathrm{H}\right)$. Mutation of the -10 region of this promoter yielded the largest drop in basal dcas9 expression (Fig. 2A), likely reflecting lower promoter activity. The RBS mutation found in strain CJW_Bb410 also decreased basal dcas9 expression levels (Fig. 2A), presumably reflecting lower stability of the 
dcas9 mRNA due to lower ribosome occupancy. Translating ribosomes are known to protect bacterial mRNAs from degradation (39). The similar levels of lacl expression among the three strains that carry the dcas9/lacl cassette on a shuttle vector (Fig. 2B) suggest that the copy number of the shuttle vector, and thus that of $d \operatorname{cas} 9$, did not vary among these strains.

Therefore, the mutations in the promoter and RBS decreased basal dcas 9 expression levels as intended.

Next, we determined appropriate induction conditions using strain CJW_Bb242 given it displayed the highest basal expression of dcas9 (Fig. 2A). In this strain, induction of dcas 9 expression with $1 \mathrm{mM}$ IPTG, a concentration known to elicit maximal expression from $\mathrm{P}_{p Q E 30}$ in B. burgdorferi (32), resulted in slower growth in liquid culture and in semisolid BSK-agarose plates when compared to the no-IPTG condition (data not shown). This growth defect is not observed during the induction of expression of other genes (32). It is therefore likely due to overproduction of dCas9 and subsequent toxic effects associated with excessive nonspecific DNA binding, as observed in other bacteria $(28,40)$. Importantly, lowering the concentration of IPTG to $0.1 \mathrm{mM}$ resulted in no discernable growth defect (Fig. 2C). We therefore used $0.1 \mathrm{mM}$ IPTG to induce dcas9 expression in all subsequent experiments.

We then measured the magnitude of induction of $d c a s 9$ expression by IPTG. The highest magnitude, 400-fold, was observed in strain CJW_Bb430, which carries a chromosomal copy of the dcas9/lacl cassette (Fig. 2D). Among the shuttle vector-encoded dcas 9 versions, we observed the highest induction of $\sim 40$-fold in the case of the unmutated promoter (Fig. 2D, strain CJW_Bb242). The RBSmut version displayed a $\sim 15$-fold induction, while the -10 promoter mutation allowed for only a two-fold induction (Fig. 2D). The genetically-linked lacl gene experienced little change in its expression level in response to IPTG induction (Fig. 2E), as expected. 
Finally, we imaged the strains after two days of exposure to IPTG and saw no notable changes in cell morphology between induced and uninduced cultures (Fig. 2F), further supporting the notion that these levels of dcas 9 induction are not toxic to the cells.

\section{B. burgdorferi genes targeted by CRISPRi.}

To test our CRISPRi platform, we targeted four B. burgdorferi genes: flaB, ftsl, rod $A$, and $m r e B$. Depletion of their protein products is expected to yield morphological phenotypes, which are easily observable by microscopy. The flaB gene, which encodes flagellin, the major structural component of periplasmic flagella, is required for motility and for generating the characteristic flat wave morphology of $B$. burgdorferi cells $(41,42)$. Ftsl is a transpeptidase that is required for septal peptidoglycan synthesis during cell division; its inhibition causes cell filamentation in E. coli (43-46). RodA, encoded by the $\operatorname{rod} A(m r d B)$ gene, is a transglycosylase active during lateral peptidoglycan synthesis in many rod-shaped bacteria (47). This lateral wall elongation is orchestrated by MreB, a bacterial actin homolog (48). When rod-shaped bacteria such as $E$. coli or $C$. crescentus lose RodA function, they grow into rounder shapes (49-51), as they do following MreB inactivation or depletion $(52,53)$.

Across them, the $f l a B$, rod $A$, mreB and $f t s /$ genes span two orders of magnitude of native expression levels (54), a range that covers a large subset of $B$. burgdorferi genes expressed during exponential growth in vitro (Fig. S2A). Furthermore, these genes are either the last gene in an operon or encode a monocistronic mRNA (Fig. S2B), rendering polar effects of CRISPRi unlikely. We designed sgRNAs targeting these four genes and cloned them either in sgRNA shuttle vectors or in all-in-one CRISPRi shuttle vectors (Table 1). These sgRNAs recognize sequences found in either the 5' UTR or the protein-coding region of the targeted gene, near the translational start site (Fig. S2B). We introduced the sgRNA-expressing shuttle 
vectors into appropriate $B$. burgdorferi host strains, generating CRISPRi strains for depletion of FlaB, Ftsl, MreB, and RodA (Table 2).

\section{Basal and induced CRISPRi activity in B. burgdorferi.}

In the absence of IPTG, clone generation, growth, and cell morphology were normal for the CRISPRi strains targeting flaB, regardless of the version of the CRISPRi platform used (Table S2). In contrast, upon transforming $B$. burgdorferi B31-A3-68 $\Delta$ bbe02::P $P_{f l g B}$-aphl (also known as "K2") (33) (Table 2) with all-in-one CRISPRi shuttle vectors containing an unmutated $\mathrm{P}_{p Q E 30}$ promoter and targeting $\operatorname{rod} A, \mathrm{mreB}$, or ftsl, we observed phenotypes consistent with significant basal CRISPRi activity (Table S2). For example, we were unable to generate clones when transforming $B$. burgdorferi $\mathrm{K} 2$ with shuttle vectors targeting mreB (Table S2). We also observed delays in appearance of colonies in BSK-agarose plates when using shuttle vectors targeting $\operatorname{rodA}$ (Table S2). Even in the absence of IPTG, cells of the RodA depletion strain (CJW_Bb346) sometimes had normal flat wave morphologies but often displayed bulging (Fig. S3), consistent with a RodA depletion phenotype. We believe that the high basal plasmidbased expression of $d c a s 9$ from the unmutated $\mathrm{P}_{p Q E 30}$ promoter (Fig. $2 \mathrm{~A}$ ), combined with the constitutive expression of the sgRNA, led to formation of enough dCas9-sgRNA complexes to repress transcription of the targeted genes even in the absence of IPTG. When targeting rodA, introduction of the -10AC1, -10AC2, or -10AC12 mutations (Fig. S1F) into the $\mathrm{P}_{p Q E 30}$ promoter in the context of the all-in-one CRISPRi shuttle vector did not fully abrogate the CRISPRi phenotype in the absence of IPTG induction (Table S2). We did not further analyze the strains carrying these constructs. In contrast, the -10TC or RBSmut versions of the all-in-one CRISPRi shuttle vector (Fig. $1 \mathrm{H}$ and Table 2), as well as the chromosomal dcas9 version of the CRISPRi platform, allowed generation of strains that displayed no or weak phenotypic evidence of basal CRISPRi activity (Table S2). 
We next characterized CRISPRi efficiency by qRT-PCR. In the absence of an sgRNA, the mRNA levels of flaB, ftsl, mreB, or rodA were not affected by IPTG addition (Fig. 3 and S4, gray bars). In the absence of IPTG, flaB mRNA levels were only slightly, if at all, reduced in strains constitutively expressing sgRNAflaB compared to the corresponding control strains lacking sgRNAflaB (Fig. 3A and S4A). In contrast, sgRNAs binding to $\mathrm{ftsl}$, mreB, or rodA decreased their targets' mRNA levels by $\sim 40 \%$ to $\sim 60 \%$ of those in the control strains, even without IPTG induction of dcas9 expression (Fig. 3B-D and S4B-D). These lower mRNA levels appeared to be relatively well tolerated by the cells, as fewer than $\sim 1 \%$ of the cells in each population displayed morphological defects based on visual inspection (Table S2).

IPTG induction of dcas 9 expression for 24 hours in three of the four strains carrying sgRNAflaB (CJW_Bb404, CJW_Bb381 and CJW_Bb313) depleted flaB mRNAs by at least $95 \%$ of the levels measured in the corresponding control strains in the absence of IPTG (Fig. 3A). The depletion was maintained over two days of IPTG exposure (Fig. S4A). Induction of dcas 9 expression in these strains also depleted lac/mRNAs (Fig. 3E and S4E). This was expected, as lacl expression is controlled by $\mathrm{P}_{\text {flaB }}$, which contains the 5' UTR of the flaB gene (7) and therefore the CRISPR site targeted by our sgRNA. In the remaining strain carrying sgRNAflaB (CJW_Bb385), flaB and lacl mRNA levels decreased noticeably less after IPTG induction (Fig. 3A, 3E, S4A, and S4E). Presumably, the weak induction of dcas9 expression from the mutated (-10TC) $P_{p Q E 30}$ promoter (Fig. 2D) is insufficient to cause repression of both the flaB and lacl genes located on the chromosome and multicopy plasmid, respectively.

We also quantified $f t s l, m r e B$, or rodA mRNA levels in the corresponding depletion strains grown in the presence of IPTG and compared them to controls lacking an sgRNA (Fig. 3B-D and S4B-D). In all strains, regardless of the version of the CRISPRi system present, induction of dcas 9 expression considerably depleted the mRNA of the targeted genes. The magnitude of the depletion after one day of IPTG exposure ranged from 95\% for rodA and $\mathrm{fts} /$ to $~ 99 \%$ for 
mreB (Fig. 3B-D). Such low mRNA levels were still observed after two days following IPTG addition to the cultures (Fig. S4B-D).

\section{Phenotypic characterization of CRISPRi-mediated flagellin depletion.}

Despite the very high level of expression of $B$. burgdorferi flagellin (Fig. S2A), CRISPRimediated depletion of this protein was able to elicit a partial loss-of-function phenotype.

Flagella impart to $B$. burgdorferi cells their motility and flat-wave morphology $(41,42)$, which we readily observed in uninduced or induced cells of control strains carrying no sgRNA (Movies S1 and S2 and Fig. 4A and S5A-C), or in uninduced cells of strains carrying sgRNAflaB (Movie S3 and Fig. 4B and S5A-D). After two days of IPTG exposure, cells of strain CJW_Bb313, which express sgRNAflaB from $\mathrm{P}_{p Q E 30}$ on the shuttle vector, displayed varying degrees of motility defects (Movies S4-S8). While most cells retained some flat-wave morphology and twitching ability, they appeared straightened compared to their control counterparts (Fig. 4B). For the cells that were still able to move, most often the movement was slowed (Movie S4), reduced to a twitching pattern (Movies S5 and S6), or localized at the cell pole region (Movie S6). Some cells displayed little to no evidence of flagellum-based motion and instead appeared to simply display Brownian motion or drift in the medium (Movies S7 and S8). Some of these cells were almost completely straight, except for the occasional kink at the division site (Movie S8).

Expressing sgRNAflaB from seven different promoters of varied strengths resulted in a similar overall straightening of the cell body (Fig. 4B, S1D, and S5D and Tables 2 and S1). Our results therefore suggest that our CRISPRi system is robust to variation in sgRNA levels in $B$. burgdorferi. We also observed an overall straightening of the cell in IPTG-induced strains that carry either the chromosomal dcas9 (CJW_Bb404, Fig. S5A) or the all-in-one CRISPRi shuttle vector with the RBS $P_{P Q E 30}$ mutation (CJW_Bb381, Fig. S5B). This is consistent with the 
significant flaB mRNA depletion observed in these strains (Fig. 3A and S4A). As expected, cells from strain CJW_Bb385, in which flaB transcript levels remained largely unaffected by IPTG induction (Fig. 3A and S4A), displayed normal morphology (Fig. S5C).

Flagellin depletion is expected to occur gradually over generations following IPTG induction, which could explain the mixed phenotypes we observed. In B. burgdorferi, multiple flagella are anchored near each cell pole $(55,56)$. They form bundles that extend from their subpolar anchors towards the center of the cell, where they overlap. We reasoned that retention of some wave-like cell shape in otherwise poorly motile or non-motile, flagellin-depleted cells may be caused by a decrease in the length or number of flagella. In this scenario, fewer flagella might exert enough force to bend the cell into a reduced-amplitude wave-like shape but not enough force to generate translational motion. To test these potential explanations, we imaged frozen-hydrated B. burgdorferi cells by cryo-electron tomography (cryo-ET). In the absence of IPTG, the cellular ultrastructure was indistinguishable from that previously reported $(56,57)$. Multiple flagella were attached near the cell poles (Fig. 4C, panel a, and Fig. S6A) and the flagellar bundles extended towards the middle of the cell, where they overlapped (Fig. 4C, panel b). In contrast, after exposure to IPTG for two days, the cells had no detectable flagellar filament around midcell (Fig. 4D and S6B, panels b), explaining the observed motility defects. At pole-proximal regions, flagellar hooks could be readily detected (Fig. 4D and S6A, panels a). However, no flagellar filament (Fig. 4D) or shorter filaments (Fig. S6A) could be detected at these subcellular regions, consistent with a strong depletion of FlaB.

\section{Ftsl involvement in B. burgdorferi cell division.}

Knockdown of $\mathrm{fts}$ / expression by CRISPRi for two days elicited significant cell filamentation in all the strains tested, in contrast to the uninduced cells of the same strains (Fig. 5A). Cell filamentation required expression of sgRNAftsl, as shown by cell length quantification (Fig. 5B). 
Cells almost $100 \mu \mathrm{m}$ long, which is about five times the average length of a $B$. burgdorferi cell, were detected (Fig. 5B, bottom). We note that when immobilized between an agarose pad and a coverslip, as in our microscopy setup, longer cells are more likely to cross themselves or other cells, bend at a tight angle, or lie partly outside the field of imaging. In such cases, automated cell outline generation with the Oufti software package (58) was not possible and such cells were excluded from our measurements. Therefore, the cell length distributions measured in Ftsl-depleted cultures likely underestimate the extent of cell filamentation present in the population. Regardless, our results implicate Ftsl in B. burgdorferi cell division.

\section{Rod morphogenesis functions of MreB and RodA in B. burgdorferi.}

We imaged the MreB and RodA depletion strains before IPTG addition and daily after induction. We found that one day of MreB depletion was enough for significant cell bulging to develop (Fig. 6A). Bulging occurred predominantly at midcell (Fig. 6A, white arrowheads). In some cells, especially long ones, bulging was also apparent at $\sim 1 / 4$ and $\sim 3 / 4$ locations along the length of the cell (Fig. 6A, blue arrowheads). Our laboratory has previously shown that new peptidoglycan synthesis occurs at these subcellular locations in members of the Borrelia genus (59). The bulging phenotype at these locations therefore suggests that MreB is important for maintaining a constant cell width during insertion of peptidoglycan material at these specific sites (48). Furthermore, less pronounced cell body widening outside of these discrete locations, but encompassing longer segments of the cells, was also observed (Fig. 6A, yellow arrowheads). After two days of MreB depletion (Fig. 6A), the bulging phenotype became further exacerbated, with larger bulges at midcell and more pronounced laterally-spread cell body widening compared to the one-day time point. Overall, our findings establish a key role for MreB in B. burgdorferi cell morphogenesis. 
RodA depletion also elicited cell widening in B. burgdorferi (Fig. 6B). In the absence of IPTG, cells looked normal (Fig. 6B). Upon IPTG induction, bulging could be observed at midcell (Fig. 6B, white arrowhead) and, occasionally, at the $1 / 4$ and $3 / 4$ locations (Fig. 6B, blue arrowheads). Midcell bulging nevertheless permitted cell division to occur in some instances, as evidenced by the occurrence of deep constriction within a bulge (Fig. 6B, pink arrowhead and inset) and the occasional presence of rounded poles (Fig. 6B, white asterisk).

\section{DISCUSSION}

In this study, we adapted the CRISPRi system for use in B. burgdorferi and tested its capabilities by targeting the motility and/or cell morphogenesis genes flaB, ftsl, mreB, and $\operatorname{rod} A$. These genes span a broad range of native expression levels (Fig. S2A) (54), suggesting that CRISPRi will have broad applicability in the study of $B$. burgdorferi.

We created several variations of the CRISPRi platform. The all-in-one CRISPRi shuttle vectors can be introduced into any transformable $B$. burgdorferi strain, allowing comparison of behaviors across multiple genetic backgrounds and facilitating the pairing of CRISPRi-based gene depletion with other genetic methods. For example, using our system, the effects of depleting one gene product can be easily compared among otherwise isogenic strains that are wild-type, mutated, or complemented at a second gene locus. We further facilitated such pairing by generating CRISPRi shuttle vectors carrying each of five compatible antibiotic resistance markers (Table 1$)$.

Another variant of our CRISPRi platform relies on two elements, a dcas9/lacl cassette stably integrated into the chromosome of a transformable derivative of the type strain B31 and an sgRNA expressed from a shuttle vector. The copy number of chromosomally expressed dcas 9 is expected to co-vary with the copy number of the targeted locus, whether it is located on the 
chromosome or on an endogenous plasmid. Prior studies have documented a $\sim 1: 1$ copy number ratio between various endogenous plasmids and the chromosome $(34,35,60)$.

A common characteristic of all versions of our CRISPRi platform is that they only require a single shuttle vector transformation step. B. burgdorferi B31-derived strains that lack restriction modification enzymes are transformed by shuttle vectors at a higher frequency, in the range $10^{-4}$ to $10^{-5}$ (33), than the $\sim 10^{-7}$ frequency obtained using suicide vectors $(7,9)$. Thus, our CRISPRi platform offers an efficient complementary tool to homologous recombination-based methods for interrogation of gene function in B. burgdorferi.

In characterizing the CRISPRi platform, we found that low basal dcas 9 expression in the absence of IPTG combined with sizeable induction of dcas9 expression upon IPTG addition appear to be important for broad functionality of the approach. Such is the case for the RBSmut version of the all-in-one CRISPRi shuttle vector and for the chromosomally-encoded dcas9. These CRISPRi platform versions yielded depletion phenotypes for all the genes we targeted. We recommend these versions in future CRISPRi experiments aimed at downregulating the expression of other genes.

Our CRISPRi approach allowed us to provide genetic insight into cell morphogenesis in $B$. burgdorferi. To our knowledge, neither deletion mutants nor transposon insertion mutants have been reported for $B$. burgdorferi ftsl, mreB, or $\operatorname{rod} A(61)$, potentially because these genes are essential for viability. Our inability to obtain clones while targeting mreB using the CRISPRi platform version that had the highest basal expression of dcas9 supports this notion for $m r e B$. We also tried to use A22 and MP265, two known small-molecule inhibitors of MreB (62-66), to study the function of this cytoskeletal protein in B. burgdorferi. Our attempts, however, proved unsuccessful, as $B$. burgdorferi appears to be resistant to chemical inhibition of MreB (see supplemental text in the combined supplemental material file and Fig. S7). Other examples of inhibitors widely used in other bacteria but not effective in $B$. burgdorferi include the 
transcription inhibitor rifampin, which acts on $\mathrm{RpoB}(67)$, and the peptidoglycan precursor synthesis inhibitor fosfomycin, which acts on MurA $(43,68)$. This list of important cellular functions that are apparently refractive to chemical inhibition in $B$. burgdorferi further underscores the utility of our easy-to-use and rapid CRISPRi genetic approach.

When we depleted Ftsl, cells elongated into filaments, consistent with a conserved involvement of this protein in cell division $(45,69,70)$. Depletion of RodA and MreB led to cell widening, reminiscent of loss-of-function phenotypes observed in other bacteria $(50,51,53,62$, 70, 71). These results fit with the current model in which MreB orients lateral cell wall synthesis in such a way that a constant cell width is generated and propagated during growth (48). RodA, in turn, is an elongation specific transglycosylase (47) that contributes to the wall biosynthetic function organized by MreB $(43,48)$. Thus, the cell widening and bulging associated with MreB and RodA depletion in B. burgdorferi indicates that rod morphogenesis is controlled in this spirochete, at least in part, by some of the same actors as in other rod-shaped bacteria.

Bulging secondary to MreB depletion overwhelmingly occurred at sites of new peptidoglycan synthesis (59), primarily the midcell, but also the $1 / 4$ and $3 / 4$ positions along the length of the cells. The pattern of new peptidoglycan insertion in Borrelia species is peculiar compared to that seen in other bacteria, including other spirochetes such as those belonging to the Treponema and Leptospira genera (59). Those spirochetes elongate by inserting new peptidoglycan along the entire length of their cells. We do not yet know what mechanisms control the Borrelia pattern of new wall insertion. However, our observation that morphologic defects secondary to MreB depletion matched sites of new peptidoglycan insertion suggests a connection between MreB function and B. burgdorferis unusual pattern of cell wall growth.

Our results highlight the usefulness of a CRISPRi genetic approach. While this approach does not replace traditional homologous recombination-based methods, it offers some advantages as a complementary approach due to its procedural ease, speed, efficiency, and 
scalability. CRISPRi-based methods have proven to be particularly useful for the phenotypic characterization of genes essential for viability, for the simultaneous repression of multiple genes, and for high-throughput genome-wide studies $(18,19,24,28)$.

\section{MATERIALS AND METHODS}

\section{Bacterial growth conditions.}

All E. coli strains were grown on LB agar plates or in Super Broth (35 g/liter bacto-tryptone, 20 $\mathrm{g} /$ liter yeast extract, $5 \mathrm{~g} /$ liter $\mathrm{NaCl}, 6 \mathrm{mM} \mathrm{NaOH}$ ) liquid cultures at $30^{\circ} \mathrm{C}$, with shaking. The following final concentrations of antibiotics were used for E. coli growth and selection: kanamycin at $50 \mu \mathrm{g} / \mathrm{mL}$, gentamicin at $15-20 \mu \mathrm{g} / \mathrm{mL}$, spectinomycin or streptomycin at 50 $\mu \mathrm{g} / \mathrm{mL}$, rifampin at $25 \mu \mathrm{g} / \mathrm{mL}$ for liquid selection or $50 \mu \mathrm{g} / \mathrm{mL}$ for growth on plates, and hygromycin B at 100-200 $\mu \mathrm{g} / \mathrm{mL}$. After heat shock or electroporation, E. coli transformants were allowed to recover in SOC medium (20 g/liter tryptone, $5 \mathrm{~g} /$ liter yeast extract, $10 \mathrm{mM}$ $\mathrm{NaCl}, 2.5 \mathrm{mM} \mathrm{KCl}, 10 \mathrm{mM} \mathrm{MgCl}_{2}, 10 \mathrm{mM} \mathrm{MgSO}_{4}, 20 \mathrm{mM}$ glucose) for 1 hour with shaking at $30^{\circ} \mathrm{C}$, before plating. E. coli strain MC1000 (72) was grown overnight in LB liquid culture at $37^{\circ} \mathrm{C}$ with shaking, then diluted 1:1000 in BSK-II medium (see below), grown for another three hours, and finally treated with 50 M MP265 or A22 (63) from a 1000X DMSO stock, or with DMSO alone, for one hour.

B. burgdorferi strains are listed in Table 2 . They were grown at $34^{\circ} \mathrm{C}$ in a humidified incubator under $5 \% \mathrm{CO}_{2}$ atmosphere $(11,13,14)$. Liquid cultures were grown in BarbourStoenner-Kelly (BSK)-II medium (11), containing $50 \mathrm{~g} /$ liter bovine serum albumin (Millipore, \#810036), 9.7 g/liter CMRL-1066 (US Biological, \#C5900-01), 5 g/liter Neopeptone (Difco \#211681), 2 g/liter Yeastolate (Difco \#255772), 6 g/liter HEPES (Millipore \#391338), 5 g/liter glucose (Sigma-Aldrich \#G7021), 2.2 g/liter sodium bicarbonate (Sigma-Aldrich \#S5761), 0.8 g/liter sodium pyruvate (Sigma-Aldrich \#P5280), $0.7 \mathrm{~g} /$ /iter sodium citrate (Fisher Scientific 
\#BP327), 0.4 g/liter N-acetylglucosamine (Sigma-Aldrich \#A3286), $\mathrm{pH} 7.60$, and $60 \mathrm{~mL} /$ liter heat inactivated rabbit serum (Gibco \#16120). BSK-1.5 medium contained $69.4 \mathrm{~g} /$ liter bovine serum albumin, $12.7 \mathrm{~g} /$ liter CMRL-1066, $6.9 \mathrm{~g} /$ liter Neopeptone, $3.5 \mathrm{~g} /$ liter Yeastolate, 8.3 g/liter HEPES, 6.9 g/liter glucose, $6.4 \mathrm{~g} /$ liter sodium bicarbonate, $1.1 \mathrm{~g} /$ liter sodium pyruvate, $1.0 \mathrm{~g} /$ /iter sodium citrate, $0.6 \mathrm{~g} /$ liter $\mathrm{N}$-acetylglucosamine, $\mathrm{pH} 7.50$, and $40 \mathrm{~mL} /$ liter heat inactivated rabbit serum. Antibiotics were used at the following final concentrations for both liquid cultures and plates: kanamycin at $200 \mu \mathrm{g} / \mathrm{mL}$, gentamicin at $40 \mu \mathrm{g} / \mathrm{mL}$, streptomycin at $100 \mu \mathrm{g} / \mathrm{mL}$, and hygromycin B at $300 \mu \mathrm{g} / \mathrm{mL}$, respectively $(6-8,73)$. IPTG (American

Bioanalytical \#AB00841) was used at $0.1 \mathrm{mM}$ final concentration from a stock of $1 \mathrm{M}$ in water. Unless otherwise specified, cultures were always maintained in exponential growth, with culture densities kept below $\sim 5 \times 10^{7}$ cells $/ \mathrm{mL}$. Cell density was determined by direct counting under darkfield illumination of samples placed in disposable hemocytometers, as previously described (6).

\section{B. burgdorferi transformation and clone isolation.}

Competent cells were generated as previously described (9). B. burgdorferi cultures containing exponentially growing cells were allowed to reach densities between $2 \times 10^{7}$ cells $/ \mathrm{mL}$ and $\sim 1 \mathrm{x}$ $10^{8} \mathrm{cells} / \mathrm{mL}$. Next, the cultures were centrifuged for $10 \mathrm{~min}$ at $10,000 \times \mathrm{g}$ and $4^{\circ} \mathrm{C}$ in $50 \mathrm{~mL}$ conical tubes, and the medium was removed by aspiration without disturbing the cell pellets. Cell pellets from 2-3 tubes were combined by resuspension in $40 \mathrm{~mL}$ cold EPS solution containing $93.1 \mathrm{~g} /$ liter sucrose (American Bioanalytical \#AB01900) and $150 \mathrm{~mL} /$ liter glycerol (American Bioanalytical \#AB00751) and centrifuged again. A second wash in $1 \mathrm{~mL}$ cold EPS was then performed, after which the cells were pelleted and resuspended in 50 to $100 \mu \mathrm{L}$ of cold EPS for each $100 \mathrm{~mL}$ of initial culture. The competent cells were placed on ice or frozen at $-80^{\circ} \mathrm{C}$ until use. 
For cell transformation with a shuttle vector $(4,74), 25$ to $50 \mu \mathrm{g}$ of plasmid (in water) were mixed with 50-100 $\mu \mathrm{L}$ of competent cells of strain B31-A3-68- $\Delta$ bbe02::P flgB $^{-a p h l ~ " K 2 " ~(33), ~ B 31 ~}$ e2 (75), or CJW_Bb362, and electroporated (2.5 kV, $25 \mu \mathrm{F}, 200 \Omega, 2 \mathrm{~mm}$ gap cuvette). Electroporated cells were immediately recovered in $6 \mathrm{~mL}$ BSK-II and incubated overnight at $34^{\circ} \mathrm{C}$. To insert the dcas9/lacl cassette into the chromosome, the pKIKan_idCas9_Chr_center plasmid $(75 \mu \mathrm{g})$ was linearized in a $500 \mu \mathrm{L}$ reaction volume containing 100 units of ApaLI enzyme for $4-6$ hours at $37^{\circ} \mathrm{C}$ in CutSmart buffer (New England Biolabs). The DNA was then ethanol precipitated as previously described (76). The DNA pellet was dried in a biosafety cabinet, then resuspended in $25 \mu \mathrm{L}$ water, chilled on ice, mixed with $100 \mu \mathrm{L}$ of competent cells of the B31-A3-68- $\Delta b b e 02:: \mathrm{P}_{f l a B}-a a d A$ "S9" strain (33), and electroporated. The cells were allowed to recover overnight in $6 \mathrm{~mL}$ of BSK-II medium.

After overnight recovery, electroporated B. burgdorferi cells were plated in semisolid BSKagarose medium. Three volumes of BSK-1.5 medium containing appropriate concentrations of antibiotics were equilibrated at $34-37^{\circ} \mathrm{C}$, then briefly brought up to $55^{\circ} \mathrm{C}$ in a water bath and mixed with two volumes of $1.7 \%(\mathrm{w} / \mathrm{v})$ agarose solution in water, also pre-equilibrated at $55^{\circ} \mathrm{C}$. The mixed BSK-antibiotics-agarose solution (25 mL/plate) was added to $10 \mathrm{~cm}$ petri dishes containing aliquots of the electroporated B. burgdorferi cells and gently swirled to mix. The plates were chilled to room temperature for $\sim 30 \mathrm{~min}$ in a biosafety cabinet, then transferred to a humidified $5 \% \mathrm{CO}_{2}$ incubator kept at $34^{\circ} \mathrm{C}$ for one to three weeks until colonies became clearly visible by eye. Alternatively, selection was performed in liquid culture by mixing $1 \mathrm{~mL}$ of electroporated cells with $5 \mathrm{~mL}$ of BSK-II medium containing appropriate concentrations of selective antibiotics. Beginning on the fifth day of selection, the cultures were visually inspected for growth by darkfield microscopy. Clones were isolated from these non-clonal, selected populations by limiting dilution in 96-well plates, as previously described (6) or by semi-solid BSK-agarose plating, as described above. Agarose plugs containing single colonies 
were removed from the plates and expanded in $6 \mathrm{~mL}$ of BSK-II medium containing selective antibiotics. Insertion of the dcas $9 / / a c /$ cassette at the desired chromosomal location in strain CJW_Bb362 was confirmed by polymerase-chain reaction (PCR) analysis of isolated total genomic DNA (DNeasy Blood \& Tissue Kit, Qiagen), using the following primer pairs: NT424 and NT425 (amplifies across the insertion site), NT591 and NT592 (amplifies within the kanamycin resistance gene aphl), NT681 and NT682 (amplifies within lacl), and NT683 and NT684 (amplifies within dcas9), respectively (see Table 3 for primer sequences). Except for strain CJW_Bb122 (which is not derived from an infectious strain), the plasmid complement of each isolated clone was determined using primer pairs previously described (77) and DreamTaq Green DNA Polymerase (ThermoFisher Scientific). Each clone was confirmed to contain all B31-specific plasmids, except Ip5, cp9, and Ip56, which are also absent from the parental strains (33). The clones were invariantly maintained in exponential growth and frozen at passage one or two.

\section{DNA manipulations.}

General methods. Standard molecular biology techniques were used to generate the plasmids listed in Table 1. Oligonucleotide primers were purchased from Integrated DNA Technologies (IDT) and their sequences are provided in Table 3. PCR was performed using Platinum Hot Start PCR Master Mix or Phusion High-Fidelity DNA polymerase (ThermoFisher Scientific). Restriction endonucleases were obtained from New England Biolabs or ThermoFisher Scientific. Gel extraction was done using PureLink Quick Gel Extraction kit (ThermoFisher Scientific). Gibson Assembly Master Mix was procured from New England Biolabs. Agilent's QuickChange Lightning Site-Directed Mutagenesis Kit was used according to the manufacturer's protocol. New England Biolabs Electroligase or Quick Ligation Kits were used. Transformations of the cloning strains DH5a (Promega), NEB 5-alpha (New England Biolabs), 
or XL-10 Gold (Agilent) were done by electroporation or heat shock. Plasmids carrying the dcas9/lacl cassette were recovered and propagated in the NEB 5-alpha F' 19 strain (New England Biolabs). Cloning and/or propagation of plasmids containing the dcas $9 / / a c /$ cassette in host $E$. coli strains that did not express lacla often led to selection of clones that carried inactivating deletions within $\mathrm{P}_{p Q E 30}$, which abrogated dcas 9 expression in both $E$. coli and $B$. burgdorferi. This was likely due to high basal expression of dcas9 in E. coli and associated non-specific toxic effects. Plasmid DNA was isolated using a Zyppy Plasmid Miniprep kit (Zymo Research) or Qiagen Plasmid Midi Kit. Correct DNA sequences of the relevant parts of the plasmids generated in this study were confirmed by Sanger sequencing at Quintarabio or at the Yale Keck Biotechnology Resource Laboratory.

Generation of sgRNA cassettes. The cloning template for an sgRNA cassette, modeled after a previous report (19), contains the following features: a promoter, a 500 bp filler sequence, a dCas9 handle region, and a transcriptional terminator sequence (Fig. 1A). To

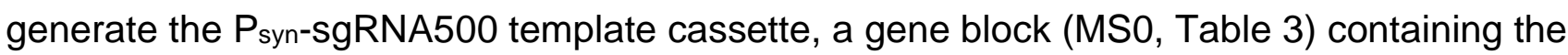
promoter $\mathrm{P}_{\text {syn, }}$ the dCas9 handle region, and the transcriptional terminator, as well as homology regions to the $B$. burgdorferi shuttle vectors, was synthesized at Integrated DNA Technologies. This gene block was Gibson-assembled with the backbones of pBSV2 (78) and pBSV2G (8), which were obtained by PCR amplification using primers MS1 and MS2, respectively. The resulting plasmids were PCR-amplified using primers MS3 and MS4, and Gibson-assembled with a 500 bp filler DNA sequence. This filler was obtained by PCR amplification of the luciferase gene of pJSB252 (32) using primers MS5 and MS6.

To place sgRNA expression under the control of constitutive $B$. burgdorferi promoters (Table S1), the following steps were taken. The sgRNA500 sequence was PCR amplified with primers NT374 and NT375, digested with BamHI and HindIII, and cloned into the BamHI and HindIII sites of shuttle vectors generated previously (6) that contain the following promoters 
between their Sacl and BamHI sites: $\mathrm{P}_{\text {flaB }}, \mathrm{P}_{\text {rest }}, \mathrm{P}_{0026}, \mathrm{P}_{0031}, \mathrm{P}_{0526}$, or $\mathrm{P}_{0826 .}$ Next, the 5' UTR contained within these promoter sequences (Table S1), as well as the BamHI site, were deleted by site-directed mutagenesis. The following primer pairs were used on the appropriate template: NT382 and NT383 generated the $\mathrm{P}_{\text {flaBs }}$-SgRNA500 cassette; NT378 and NT379

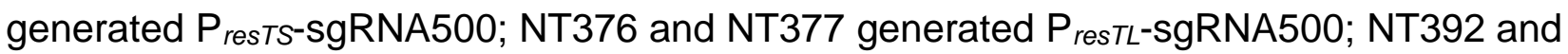
NT393 generated Po826S-sgRNA500; NT390 and NT391 generated Po826L-sgRNA500; NT384 and NT385 generated P0026-sgRNA500; NT388 and NT389 generated P0526-SgRNA500, and NT386 and NT387 generated P0031-sgRNA500.

To generate mature sgRNA cassettes targeting specific genes, the 500-bp filler was excised from plasmids containing the template sgRNA500 cassette by digestion with Sapl, BspQI, or Lgul. Pairs of primers (Tables 3 and 4) were annealed, generating a short dsDNA sequence with overhangs complementary to the overhangs generated by Sapl, BspQI, or Lgul digestion of the plasmid. The digested plasmid and annealed primers were ligated. To obtain pBSV2G_PflaBs-sgRNAflaB, site-directed mutagenesis was performed on pBSV2G_PflaBSsgRNA500 using primers NT409 and NT410.

Choice of sgRNA target base-pairing sequence. The coding sequence of a gene of interest, in the $5^{\prime}$ to $3^{\prime}$ orientation and including $\sim 100$ bp upstream of the gene (to ensure that the $5^{\prime}$ UTR, if present, was included), was imported into the Geneious R10 software package. CRISPR sites were then identified using the "Find CRISPR sites" feature of the software. The required parameters were a 20-nucleotide base pairing region upstream of an NGG-3' PAM site. CRISPR target sites were selected for further evaluation based on the following criteria: (i) they mapped within the target gene's 5' UTR or within its protein-coding region close to the translational start site and (ii) their orientation was opposite that of the coding region, thus ensuring that the sgRNA targets the non-template strand $(18,19)$. Next, the NCBI webtool blastn was used to compare the CRISPR target site sequence against the complete $B$. 
burgdorferi B31 genome sequence (79) to rule out off-target binding. Primer pairs were then designed to encode sequences complementary to the CRISPR target sites, minus the PAM. A guanine base was added 5' to the base-pairing sequence to ensure similar efficiency of transcription of the various sgRNAs and to account for the purine preference at the +1 position of the TSS observed across several bacterial genomes $(80,81)$. Finally, the primers were designed to also generate, upon annealing, overhangs compatible with the Sapl-digested plasmids containing template sgRNA cassettes.

Transfer of sgRNA cassettes. Among the shuttle vectors that do not contain the dcas9/lacl cassette, sgRNA cassettes were transferred as Sacl/Fspl restriction fragments. $\mathrm{P}_{\text {syn-containing }}$ cassettes (e.g., Psyn-sgRNAflaB) were inserted into pBbdCas9 vectors as Ascl/Eagl digests of PCR products generated using primers NT354 and NT355 and the appropriate template DNA. All other cassettes were inserted into pBbdCas9 vectors as Ascl/Eagl digests of PCR products generated using primers NT355 and NT449. The sgRNA cassettes were transferred among various pBbdCas9 vectors (see below) as either Ascl/Eagl or Eagl/Xmal digests.

Updated sequence of $\mathrm{pBSV} 2 \mathrm{H}$. During this work, we discovered that $\mathrm{pBSV} 2 \mathrm{H}$, which we generated as part of our previous study (6), contains a duplication of its dual rifampinhygromycin B resistance cassette. This was confirmed by quality control tests performed at Addgene. We have therefore updated the sequence of the construct on Addgene's product page (\#118229). All pBSV2H-derived plasmids generated in this study (Table 1) also contain this duplication. However, based on the normal behavior of the B. burgdorferi strains generated with these plasmids (see results obtained using chromosomal dcas 9 CRISPRi strains), we believe that the antibiotic cassette duplication does not affect the functionality of these vectors. Nevertheless, we generated a version of this shuttle vector that carries only one copy of the dual antibiotic resistance cassette, which we named pBSV2H_2 (Table 1). 
Generation of all-in-one CRISPRi shuttle vectors. The luciferase gene of pJSB252 (32) was replaced with dcas9 as follows. The pJSB252 backbone was PCR-amplified with primers MS11 and MS12. The dcas9 gene encoding the catalytically inactive protein (18) was PCRamplified from plasmid pdCas9-bacteria using primers MS13 and MS14. The two fragments were Gibson-assembled. Next, a silent mutation was introduced into the lacl gene to remove the Sapl site. This was done by site-directed mutagenesis using NT352 and NT353, yielding plasmid pBbdCas9S. We note that the dcas9 gene present in our constructs carries a mutation that results in a M1169L amino acid change, but dcas9 rermains functional despite this change. The arr2 rifampin-resistance gene was then PCR-amplified from pBSV2B (6) using primers NT170 and NT446, digested with Ascl and Eagl, and cloned into the Ascl/Eagl backbone of

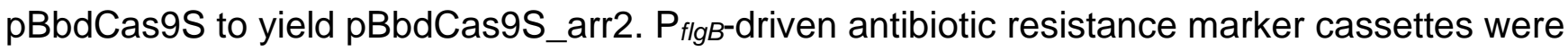
generated as follows: primers NT698 and NT702 were used to PCR amplify the kanamycin cassette of pBSV2_2; NT699 and NT702 were used to amplify the gentamicin cassette of pBSV2G_2; NT700 and NT702 were used to amplify the blasticidin S cassette of pBSV2B; and NT701 and NT702 were used to amplify the hygromycin B cassette of pBSV2H. The resulting PCR products were digested with BamHI and Aatll and ligated into the backbone of pBbdCas9S_arr2 obtained following sequential Bglll and Aatll digestion. This process generated the following plasmids: pBbdCas9K_arr2 (kanamycin resistant), pBbdCas9G_arr2 (gentamicin resistant), pBbdCas9B_arr2 (blasticidin S resistant), and pBbdCas9H_arr2 (hygromycin B resistant) (Table 1).

Altered regulation of $d \operatorname{cas} 9$ expression. The following primer pairs were used to modify, by site-directed mutagenesis, the DNA region upstream of the dcas 9 coding sequence. NT669 and NT670 were used to mutate the ribosomal binding site, generating RBSmut constructs (Fig. $1 \mathrm{H})$. The rest of the mutagenesis reactions modified the -10 region of the dcas 9 promoter,

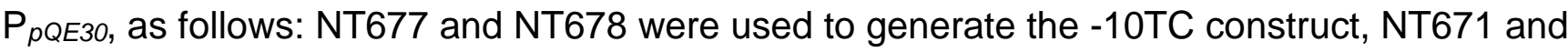


NT672 for the -10AC1 construct, NT673 and NT674 for the -10AC2 construct, and NT675 and NT676 for the -10AC12 construct (Fig. 1H and S1F).

Suicide vector for chromosome integration of the dcas9/lacl cassette. The following gene segments were assembled through a series of intermediate constructs. (i) The aphl gene of pCR2.1 TOPO was deleted by Gibson assembly using primers MS15 and MS16. The bla gene of the resulting backbone was deleted by site-directed mutagenesis using primers NT89 and NT90. The resulting backbone retains the E. coli origin of replication of $\mathrm{pCR} 2.1$ and its multicloning site. (ii) The antibiotic resistance cassette was assembled into this backbone by linking $\mathrm{P}_{\text {flgB }}$ (flanked by Sacll and Ndel sites), the aphl gene (flanked by Ndel and Xmal sites), and the flaB terminator, obtained by annealing primers NT350 and NT351 and inserting them between sites Spel and Xmal. (iii) The sequence from nucleotide 474180 to nucleotide 476218 of the B31 chromosome was amplified with primers NT267 and NT268 and cloned as a Sacl/Spel fragment. (iv) The sequence from nucleotide 476251 to nucleotide 478279 of the B31 chromosome was amplified with primers NT269 and NT270 and cloned as a Pst//Xhol fragment. The backbone, antibiotic resistance cassette, and the two homology regions allow insertion by double cross-over into the B31 chromosome between nucleotide 476218 and nucleotide 476251 in the intergenic region between genes $b b 0456$ and $b b 0457$. This suicide vector sequence was amplified with primers NT593 and NT594, while the dcas9/lacl cassette was PCR-amplified using primers NT595 and NT596 from pBbdCas9S_PresTs-sgRNAflaB. These two PCR products were Gibson-assembled to yield pKIKan_idCas9_Chr_center. pBSV2 Psyn-mCherry ${ }^{\mathrm{Bb}}$. Primers NT394 and NT395 were annealed and ligated into the Sacl/BamHI backbone of pBSV2_PresT-mCherry ${ }^{\mathrm{Bb}}(6)$. 


\section{RNA isolation and qRT-PCR.}

Exponentially growing cultures of the CRISPRi strains were counted and diluted to $10^{6}$ cells $/ \mathrm{mL}$ for next-day harvesting, or to $10^{5}$ cells $/ \mathrm{mL}$ for day-two harvesting. For situations in which IPTG induction caused growth defects, the culture induced for two days was also started at a density of $10^{6}$ cells $/ \mathrm{mL}$ to ensure that similar amounts of RNA were obtained as in the non-induced culture at the time of harvest. Dilutions were carried out in $25 \mathrm{~mL}$ of BSK-II medium with or without $0.1 \mathrm{mM}$ IPTG. At 24 or 48 hours post-induction, bacteria were harvested by centrifugation of the $25 \mathrm{~mL}$ culture for 10 minutes at $4300 \mathrm{x} \mathrm{g}$ and room temperature in a swinging bucket centrifuge. Supernatant was aspirated and the pellet was resuspended in 400-500 $\mu \mathrm{L} \mathrm{HN}$ buffer (50 mM NaCl, $10 \mathrm{mM} \mathrm{HEPES,} \mathrm{pH} \mathrm{8.0)} \mathrm{(82).} \mathrm{The}$ suspension was then immediately added to $1 \mathrm{~mL}$ RNAprotect Bacteria Reagent (Qiagen), vortexed, incubated for 5 minutes at room temperature, and centrifuged at 10,000 x g and room temperature for 10 minutes. The supernatant was aspirated and the pellet was stored at $80^{\circ} \mathrm{C}$ until RNA could be extracted using an enzymatic lysis and proteinase $\mathrm{K}$ digestion method (Protocol 4 in the RNAprotect Bacteria Reagent manual), followed by purification using the RNeasy Mini kit (Qiagen). A DNase digestion step was then performed using the TURBO DNA-free kit (ThermoFisher Scientific) and purified RNA was stored at $-80^{\circ} \mathrm{C}$.

The KAPA SYBR FAST One-Step qRT-PCR Master Mix Kit was used to quantify transcript levels. Reactions were done in duplicate or triplicate, using $10 \mathrm{ng}$ total RNA per reaction. At least one additional reaction was performed for each sample without the addition of the reverse transcriptase mix. The $\mathrm{Cq}$ values obtained from these control reactions confirmed that none of our results could be explained by DNA contamination of the RNA sample. The following protocol was used on a Bio-Rad iCycler: reverse transcription (5 min at $\left.42^{\circ} \mathrm{C}\right)$; enzyme activation ( 3 min at $95^{\circ} \mathrm{C}$ ); 40 cycles of annealing, extension, and data acquisition ( 3 $\sec$ at $95^{\circ} \mathrm{C}, 20 \mathrm{sec}$ at $\left.60^{\circ} \mathrm{C}\right)$; and a melt curve analysis $\left(55\right.$ to $95^{\circ} \mathrm{C}$ in $0.5^{\circ} \mathrm{C}$ increments). The 
primers used to PCR amplify the various targets are provided in Table 5 . The amount of each transcript of interest was normalized to the level of recA transcript in the same sample, and then expressed relative to the level of target transcript in the control sample, as previously described (83).

\section{Microscopy.}

Routine darkfield imaging of cultures was accomplished using a Nikon 40X 0.55 numerical aperture (NA) Ph2 phase-contrast air objective mounted on a Nikon Eclipse E600 microscope equipped with a darkfield condenser ring. Darkfield images and movies were acquired on a Nikon Eclipse Ti microscope equipped with a 40X 0.60 NA objective, a Nikon dry darkfield condenser (0.80-0.95 NA), and a Hamamatsu Orca-Flash4.0 V2 digital complementary metaloxide semiconductor (CMOS) camera. The same microscope was used to obtain phase contrast images using a 100X Plan Apo 1.45 NA Ph3 phase-contrast oil immersion objective and a Ph3 condenser ring. The microscope was controlled using the Nikon Elements software. Fluorescence imaging of strain CJW_Bb122 was done on a Nikon Eclipse Ti microscope with the following features: a 100X Plan Apo 1.40 NA Ph3 phase-contrast oil objective, a Hamamatsu Orca-Flash4.0 V2 CMOS camera, a Sola light engine (Lumencor), an mCherry/TexasRed fluorescence filter cube containing an ET560/40x excitation filter, a T585lpxr dichroic mirror, and an ET630/75m emission filter (Chroma), and Metamorph software (Molecular Devices). Images were processed using Nikon Elements Software, Metamorph software, or Fiji software (84).

\section{Image analysis.}

Cell outlines were generated based on phase-contrast images using Oufti, our open-source analysis software package (58). The raw outlines were curated as follows: (i) outlines assigned 
to cells that crossed other cells, folded upon themselves, or were only partially present in the imaged frame, as well as outlines assigned to image components other than cells, were manually removed; (ii) outlines were manually extended to the tips of the cells where appropriate; (iii) outlines were manually added to cells whose geometry could support an outline but whose outlines were not generated automatically by Oufti; and (iv) when clear dips in the phase signal indicated an outer membrane bridge between two cytoplasmic cylinders that had separated as part of the cell division process, the two sides of the cell were treated as independent cellular units. In such cases, separate outlines generated by Oufti were left in place, while single outlines were manually split at the point at which the phase signal was observed to dip. Finally, all outlines were refined using the Refine ALL function of the Oufti software, and the fluorescence signal data was added to the outlines. Fluorescence quantification was done as previously described, using the MATLAB script addMeshtoCellList.m and the function CalculateFluorPerCell.m (6). Cell lengths were extracted using the MATLAB script get_um_lengths.m (85), and values below $1 \mu \mathrm{m}$ were excluded from the analysis.

\section{Cryo-electron tomography and three-dimensional visualization.}

B. burgdorferi cultures growing exponentially in BSK-II medium to no more that $5 \times 10^{7}$ cells $/ \mathrm{mL}$ were pelleted for 10 minutes at $3,000 \times \mathrm{g}$ at room temperature. The pellet was gently resuspended in a small volume (50-100 $\mu \mathrm{L})$ of BSK-H (Sigma-Aldrich, \#B8291). The cell suspension was then mixed with $10-\mathrm{nm}$ colloidal gold fiducial markers. Five microliters of cell suspension were deposited on holey carbon electron microscopy grids (200 mesh, R2/1, Quantifoil), which had been freshly glow-discharged for $~ 30$ seconds. The grids were then blotted with filter paper and rapidly frozen in liquid ethane using a homemade gravity-driven plunger apparatus. The frozen-hydrated specimens were transferred to a $300 \mathrm{kV}$ Titan Krios 
electron microscope (ThermoFisher Scientific) equipped with a K2 Direct Electron Detector and energy filter (Gatan). SerialEM (86) was used to collect single-axis tilt series around $-5 \mu \mathrm{m}$ defocus, with a cumulative dose of $\sim 70$ e-/Å covering angles from $-51^{\circ}$ to $51^{\circ}$ with a $3^{\circ}$ tilt step. Images were acquired at $26,000 X$ magnification with an effective pixel size of $5.457 \AA$ at the specimen level. All recorded images were first drift corrected using the MotionCor2 software (87) and then stacked by the software package IMOD (88). In total, 11 tilt series were aligned and reconstructed using IMOD. Three-dimensional models of the flagella and cells were manually segmented and visualized by IMOD.

\section{DATA AVAILABILITY AND ACCESSION NUMBERS}

Plasmids generated in this study (and their sequences) are available through Addgene under the accession numbers listed in Table 1. Strain CJW_Bb362 will be made available through ATCC's BEI Resources collection (Item \# NR-53512). Reasonable requests for all other $B$. burgdorferi strains generated in this study shall be honored by the Jacobs-Wagner lab. The MATLAB code used to process cell fluorescence data can be downloaded from GitHub (85). MATLAB code, including dependencies, are provided at GitHub under

\section{https://github.com/JacobsWagnerLab/published.}

\section{SUPPLEMENTAL MATERIAL}

Supplemental material includes supplemental text, supplemental figures S1 to S7, supplemental tables S1 and S2, and supplemental movies S1 to S8.

\section{ACKNOWLEDGEMENTS}

We thank Dr. Brandon Jutras for providing an early intermediate plasmid used in the generation of the pKIKan_idCas9_Chr_center plasmid, Nicholas Jannetty for assistance in 
generating pBSV2_Psyn-mCherry ${ }^{\mathrm{Bb}}$, and the members of the Jacobs-Wagner lab for critical reading of the manuscript. We also thank Dr. Jon Blevins (University of Arkansas for Medical Sciences) for sharing the pJSB252 plasmid and Dr. Will Arnold (Addgene) for sharing the quality control data on plasmid pBSV2H.

\section{FUNDING}

C.J.-W. is an Investigator of the Howard Hughes Medical Institute, which supported this work.

C.N.T. was supported in part by an American Heart Association postdoctoral fellowship (award number 18POST33990330). P.A.R. is a Senior Investigator supported by the Intramural Research Program of the National Institute of Allergy and Infectious Diseases, National Institutes of Health and contributed to this work while a Visiting Fellow in the C.J.-W. laboratory at Yale University. Z.A.K. was supported by the Medical Scientist Training Grant T32 GM007205 from the National Institute of General Medical Sciences, National Institutes of Health. J.L. and Y.C. were supported by grants R01Al087946 and R01Al132818 from the National Institute of Allergy and Infectious Disease, National Institutes of Health. The funders had no role in study design, data collection and interpretation, or the decision to submit the work for publication.

\section{CONFLICT OF INTEREST}

The authors are aware of no conflict of interest.

\section{REFERENCES}

1. Radolf JD, Caimano MJ, Stevenson B, Hu LT. 2012. Of ticks, mice and men: understanding the dual-host lifestyle of Lyme disease spirochaetes. Nat Rev Microbiol 10:87-99.

2. Mead PS. 2015. Epidemiology of Lyme disease. Infect Dis Clin North Am 29:187-210. 
3. Steere AC, Strle F, Wormser GP, Hu LT, Branda JA, Hovius JW, Li X, Mead PS. 2016. Lyme borreliosis. Nat Rev Dis Primers 2:16090.

4. Samuels DS, Drecktrah D, Hall LS. 2018. Genetic transformation and complementation. Methods Mol Biol 1690:183-200.

5. Drecktrah D, Samuels DS. 2018. Genetic manipulation of Borrelia spp. Curr Top Microbiol Immunol 415:113-140.

6. Takacs CN, Kloos ZA, Scott M, Rosa PA, Jacobs-Wagner C. 2018. Fluorescent proteins, promoters, and selectable markers for applications in the Lyme disease spirochete Borrelia burgdorferi. Appl Environ Microbiol 84:e01824-18.

7. Bono JL, Elias AF, Kupko JJ, 3rd, Stevenson B, Tilly K, Rosa P. 2000. Efficient targeted mutagenesis in Borrelia burgdorferi. J Bacteriol 182:2445-2452.

8. Elias AF, Bono JL, Kupko JJ, 3rd, Stewart PE, Krum JG, Rosa PA. 2003. New antibiotic resistance cassettes suitable for genetic studies in Borrelia burgdorferi. J Mol Microbiol Biotechnol 6:29-40.

9. Tilly K, Elias AF, Bono JL, Stewart P, Rosa P. 2000. DNA exchange and insertional inactivation in spirochetes. J Mol Microbiol Biotechnol 2:433-442.

10. Criswell D, Tobiason VL, Lodmell JS, Samuels DS. 2006. Mutations conferring aminoglycoside and spectinomycin resistance in Borrelia burgdorferi. Antimicrob Agents Chemother 50:445-452.

11. Barbour AG. 1984. Isolation and cultivation of Lyme disease spirochetes. Yale J Biol Med 57:521525.

12. Elias AF, Stewart PE, Grimm D, Caimano MJ, Eggers CH, Tilly K, Bono JL, Akins DR, Radolf JD, Schwan TG, Rosa P. 2002. Clonal polymorphism of Borrelia burgdorferi strain B31 MI: implications for mutagenesis in an infectious strain background. Infect Immun 70:2139-2150. 13. Zuckert WR. 2007. Laboratory maintenance of Borrelia burgdorferi. Curr Protoc Microbiol Chapter 12:Unit 12C 11. 
14. Jutras BL, Chenail AM, Stevenson B. 2013. Changes in bacterial growth rate govern expression of the Borrelia burgdorferi OspC and Erp infection-associated surface proteins. J Bacteriol 195:757764.

15. Elias A, Bono JL, Tilly K, Rosa P. 1998. Growth of infectious and non-infectious B. burgdorferi at different salt concentrations. Wien Klin Wochenschr 110:863-865.

16. Heroldova M, Nemec M, Hubalek Z. 1998. Growth parameters of Borrelia burgdorferi sensu stricto at various temperatures. Zentralbl Bakteriol 288:451-455.

17. Latham JI, Blevins JS. 2018. Generation of conditional mutants in Borrelia burgdorferi. Methods Mol Biol 1690:225-239.

18. Qi LS, Larson MH, Gilbert LA, Doudna JA, Weissman JS, Arkin AP, Lim WA. 2013. Repurposing CRISPR as an RNA-guided platform for sequence-specific control of gene expression. Cell 152:1173-1183.

19. Larson MH, Gilbert LA, Wang X, Lim WA, Weissman JS, Qi LS. 2013. CRISPR interference (CRISPRi) for sequence-specific control of gene expression. Nat Protoc 8:2180-2196.

20. Jinek M, Chylinski K, Fonfara I, Hauer M, Doudna JA, Charpentier E. 2012. A programmable dual-RNA-guided DNA endonuclease in adaptive bacterial immunity. Science 337:816-821.

21. Koonin EV, Makarova KS, Zhang F. 2017. Diversity, classification and evolution of CRISPR-Cas systems. Curr Opin Microbiol 37:67-78.

22. Jones DL, Leroy P, Unoson C, Fange D, Curic V, Lawson MJ, Elf J. 2017. Kinetics of dCas9 target search in Escherichia coli. Science 357:1420-1424.

23. Gilbert LA, Larson MH, Morsut L, Liu Z, Brar GA, Torres SE, Stern-Ginossar N, Brandman O, Whitehead EH, Doudna JA, Lim WA, Weissman JS, Qi LS. 2013. CRISPR-mediated modular RNA-guided regulation of transcription in eukaryotes. Cell 154:442-451.

24.Peters JM, Colavin A, Shi H, Czarny TL, Larson MH, Wong S, Hawkins JS, Lu CHS, Koo BM, Marta E, Shiver AL, Whitehead EH, Weissman JS, Brown ED, Qi LS, Huang KC, Gross CA. 
2016. A comprehensive, CRISPR-based functional analysis of essential genes in bacteria. Cell 165:1493-1506.

25. Westbrook AW, Moo-Young M, Chou CP. 2016. Development of a CRISPR-Cas9 tool kit for comprehensive engineering of Bacillus subtilis. Appl Environ Microbiol 82:4876-4895.

26. Irnov I, Wang Z, Jannetty ND, Bustamante JA, Rhee KY, Jacobs-Wagner C. 2017. Crosstalk between the tricarboxylic acid cycle and peptidoglycan synthesis in Caulobacter crescentus through the homeostatic control of alpha-ketoglutarate. PLoS Genet 13:e1006978.

27. Guzzo M, Castro LK, Reisch CR, Guo MS, Laub MT. 2020. A CRISPR interference system for efficient and rapid gene knockdown in Caulobacter crescentus. mBio 11.

\section{Rock JM, Hopkins FF, Chavez A, Diallo M, Chase MR, Gerrick ER, Pritchard JR, Church} GM, Rubin EJ, Sassetti CM, Schnappinger D, Fortune SM. 2017. Programmable transcriptional repression in mycobacteria using an orthogonal CRISPR interference platform. Nat Microbiol 2:16274.

29. Singh AK, Carette X, Potluri LP, Sharp JD, Xu R, Prisic S, Husson RN. 2016. Investigating essential gene function in Mycobacterium tuberculosis using an efficient CRISPR interference system. Nucleic Acids Res 44:e143.

30. Choudhary E, Lunge A, Agarwal N. 2016. Strategies of genome editing in mycobacteria: Achievements and challenges. Tuberculosis (Edinb) 98:132-138.

31. Fernandes LGV, Guaman LP, Vasconcellos SA, Heinemann MB, Picardeau M, Nascimento A. 2019. Gene silencing based on RNA-guided catalytically inactive Cas9 (dCas9): a new tool for genetic engineering in Leptospira. Sci Rep 9:1839.

32. Blevins JS, Revel AT, Smith AH, Bachlani GN, Norgard MV. 2007. Adaptation of a luciferase gene reporter and lac expression system to Borrelia burgdorferi. Appl Environ Microbiol 73:15011513. 
33. Rego RO, Bestor A, Rosa PA. 2011. Defining the plasmid-borne restriction-modification systems of the Lyme disease spirochete Borrelia burgdorferi. J Bacteriol 193:1161-1171.

34. Kasumba IN, Bestor A, Tilly K, Rosa PA. 2015. Use of an endogenous plasmid locus for stable in trans complementation in Borrelia burgdorferi. Appl Environ Microbiol 81:1038-1046.

\section{Tilly K, Krum JG, Bestor A, Jewett MW, Grimm D, Bueschel D, Byram R, Dorward D,} Vanraden MJ, Stewart P, Rosa P. 2006. Borrelia burgdorferi OspC protein required exclusively in a crucial early stage of mammalian infection. Infect Immun 74:3554-3564.

36. Beaurepaire C, Chaconas G. 2007. Topology-dependent transcription in linear and circular plasmids of the segmented genome of Borrelia burgdorferi. Mol Microbiol 63:443-453.

37. Alverson J, Bundle SF, Sohaskey CD, Lybecker MC, Samuels DS. 2003. Transcriptional regulation of the $\operatorname{sp} A B$ and $\operatorname{sp} C$ promoters from Borrelia burgdorferi. Mol Microbiol 48:16651677.

38. Alverson J, Samuels DS. 2002. groEL expression in gyrB mutants of Borrelia burgdorferi. J Bacteriol 184:6069-6072.

39. Deana A, Belasco JG. 2005. Lost in translation: the influence of ribosomes on bacterial mRNA decay. Genes Dev 19:2526-2533.

40. Cho S, Choe D, Lee E, Kim SC, Palsson B, Cho BK. 2018. High-level dCas9 expression induces abnormal cell morphology in Escherichia coli. ACS Synth Biol 7:1085-1094.

41. Motaleb MA, Corum L, Bono JL, Elias AF, Rosa P, Samuels DS, Charon NW. 2000. Borrelia burgdorferi periplasmic flagella have both skeletal and motility functions. Proc Natl Acad Sci U S A 97:10899-10904.

42. Goldstein SF, Charon NW, Kreiling JA. 1994. Borrelia burgdorferi swims with a planar waveform similar to that of eukaryotic flagella. Proc Natl Acad Sci U S A 91:3433-3437.

43. Typas A, Banzhaf M, Gross CA, Vollmer W. 2012. From the regulation of peptidoglycan synthesis to bacterial growth and morphology. Nat Rev Microbiol 10:123-136. 
44. Botta GA, Park JT. 1981. Evidence for involvement of penicillin-binding protein 3 in murein synthesis during septation but not during cell elongation. J Bacteriol 145:333-340.

45. Spratt BG. 1977. Temperature-sensitive cell division mutants of Escherichia coli with thermolabile penicillin-binding proteins. J Bacteriol 131:293-305.

46. Tamura T, Suzuki H, Nishimura Y, Mizoguchi J, Hirota Y. 1980. On the process of cellular division in Escherichia coli: isolation and characterization of penicillin-binding proteins 1a, 1b, and 3. Proc Natl Acad Sci U S A 77:4499-4503.

47. Meeske AJ, Riley EP, Robins WP, Uehara T, Mekalanos JJ, Kahne D, Walker S, Kruse AC, Bernhardt TG, Rudner DZ. 2016. SEDS proteins are a widespread family of bacterial cell wall polymerases. Nature 537:634-638.

48. Shi H, Bratton BP, Gitai Z, Huang KC. 2018. How to build a bacterial cell: MreB as the foreman of E. coli construction. Cell 172:1294-1305.

49. Matsuzawa H, Hayakawa K, Sato T, Imahori K. 1973. Characterization and genetic analysis of a mutant of Escherichia coli K-12 with rounded morphology. J Bacteriol 115:436-442.

50. Bendezu FO, de Boer PA. 2008. Conditional lethality, division defects, membrane involution, and endocytosis in mre and $m r d$ shape mutants of Escherichia coli. J Bacteriol 190:1792-1811.

51. Wagner JK, Galvani CD, Brun YV. 2005. Caulobacter crescentus requires RodA and MreB for stalk synthesis and prevention of ectopic pole formation. J Bacteriol 187:544-553.

52. Wachi M, Matsuhashi M. 1989. Negative control of cell division by $m r e B$, a gene that functions in determining the rod shape of Escherichia coli cells. J Bacteriol 171:3123-3127.

53. Figge RM, Divakaruni AV, Gober JW. 2004. MreB, the cell shape-determining bacterial actin homologue, co-ordinates cell wall morphogenesis in Caulobacter crescentus. Mol Microbiol 51:1321-1332. 
54. Arnold WK, Savage CR, Brissette CA, Seshu J, Livny J, Stevenson B. 2016. RNA-seq of Borrelia burgdorferi in multiple phases of growth reveals insights into the dynamics of gene expression, transcriptome architecture, and noncoding RNAs. PLoS One 11:e0164165.

55. Hovind-Hougen K. 1984. Ultrastructure of spirochetes isolated from Ixodes ricinus and Ixodes dammini. Yale J Biol Med 57:543-548.

56. Kudryashev M, Cyrklaff M, Baumeister W, Simon MM, Wallich R, Frischknecht F. 2009. Comparative cryo-electron tomography of pathogenic Lyme disease spirochetes. Mol Microbiol 71:1415-1434.

57. Zhang K, He J, Cantalano C, Guo Y, Liu J, Li C. 2020. FlhF regulates the number and configuration of periplasmic flagella in Borrelia burgdorferi. Mol Microbiol 113:1122-1139.

58. Paintdakhi A, Parry B, Campos M, Irnov I, Elf J, Surovtsev I, Jacobs-Wagner C. 2016. Oufti: an integrated software package for high-accuracy, high-throughput quantitative microscopy analysis. Mol Microbiol 99:767-777.

59. Jutras BL, Scott M, Parry B, Biboy J, Gray J, Vollmer W, Jacobs-Wagner C. 2016. Lyme disease and relapsing fever Borrelia elongate through zones of peptidoglycan synthesis that mark division sites of daughter cells. Proc Natl Acad Sci U S A 113:9162-9170.

60. Hinnebusch J, Barbour AG. 1992. Linear- and circular-plasmid copy numbers in Borrelia burgdorferi. J Bacteriol 174:5251-5257.

61. Lin T, Gao L, Zhang C, Odeh E, Jacobs MB, Coutte L, Chaconas G, Philipp MT, Norris SJ. 2012. Analysis of an ordered, comprehensive STM mutant library in infectious Borrelia burgdorferi: insights into the genes required for mouse infectivity. PLoS One 7:e47532.

62. Gitai Z, Dye NA, Reisenauer A, Wachi M, Shapiro L. 2005. MreB actin-mediated segregation of a specific region of a bacterial chromosome. Cell 120:329-341. 
63. Takacs CN, Poggio S, Charbon G, Pucheault M, Vollmer W, Jacobs-Wagner C. 2010. MreB drives de novo rod morphogenesis in Caulobacter crescentus via remodeling of the cell wall. $\mathrm{J}$ Bacteriol 192:1671-1684.

64. Iwai N, Nagai K, Wachi M. 2002. Novel S-benzylisothiourea compound that induces spherical cells in Escherichia coli probably by acting on a rod-shape-determining protein(s) other than penicillin-binding protein 2. Biosci Biotechnol Biochem 66:2658-2662.

65. Iwai N, Ebata T, Nagura H, Kitazume T, Nagai K, Wachi M. 2004. Structure-activity relationship of S-benzylisothiourea derivatives to induce spherical cells in Escherichia coli. Biosci Biotechnol Biochem 68:2265-2269.

66. Iwai N, Fujii T, Nagura H, Wachi M, Kitazume T. 2007. Structure-activity relationship study of the bacterial actin-like protein MreB inhibitors: effects of substitution of benzyl group in Sbenzylisothiourea. Biosci Biotechnol Biochem 71:246-248.

67. Alekshun M, Kashlev M, Schwartz I. 1997. Molecular cloning and characterization of Borrelia burgdorferi rpoB. Gene 186:227-235.

68. Jiang S, Gilpin ME, Attia M, Ting YL, Berti PJ. 2011. Lyme disease enolpyruvyl-UDP-GlcNAc synthase: fosfomycin-resistant MurA from Borrelia burgdorferi, a fosfomycin-sensitive mutant, and the catalytic role of the active site Asp. Biochemistry 50:2205-2212.

69. Ohta N, Ninfa AJ, Allaire A, Kulick L, Newton A. 1997. Identification, characterization, and chromosomal organization of cell division cycle genes in Caulobacter crescentus. J Bacteriol 179:2169-2180.

70. Slamti L, de Pedro MA, Guichet E, Picardeau M. 2011. Deciphering morphological determinants of the helix-shaped Leptospira. J Bacteriol 193:6266-6275.

71. Jones LJ, Carballido-Lopez R, Errington J. 2001. Control of cell shape in bacteria: helical, actinlike filaments in Bacillus subtilis. Cell 104:913-922. 
72. Casadaban MJ, Cohen SN. 1980. Analysis of gene control signals by DNA fusion and cloning in Escherichia coli. J Mol Biol 138:179-207.

73. Frank KL, Bundle SF, Kresge ME, Eggers CH, Samuels DS. 2003. a adA confers streptomycin resistance in Borrelia burgdorferi. J Bacteriol 185:6723-6727.

74. Samuels DS. 1995. Electrotransformation of the spirochete Borrelia burgdorferi. Methods Mol Biol 47:253-259.

75. Casjens S, van Vugt R, Tilly K, Rosa PA, Stevenson B. 1997. Homology throughout the multiple 32-kilobase circular plasmids present in Lyme disease spirochetes. J Bacteriol 179:217-227.

76. Green MR, Sambrook J. 2016. Precipitation of DNA with ethanol. Cold Spring Harb Protoc 2016:1116-1120.

77. Bunikis I, Kutschan-Bunikis S, Bonde M, Bergstrom S. 2011. Multiplex PCR as a tool for validating plasmid content of Borrelia burgdorferi. J Microbiol Methods 86:243-247.

78. Stewart PE, Thalken R, Bono JL, Rosa P. 2001. Isolation of a circular plasmid region sufficient for autonomous replication and transformation of infectious Borrelia burgdorferi. Mol Microbiol 39:714-721.

79. Fraser CM, Casjens S, Huang WM, Sutton GG, Clayton R, Lathigra R, White O, Ketchum KA, Dodson R, Hickey EK, Gwinn M, Dougherty B, Tomb JF, Fleischmann RD, Richardson D, Peterson J, Kerlavage AR, Quackenbush J, Salzberg S, Hanson M, van Vugt R, Palmer N, Adams MD, Gocayne J, Weidman J, Utterback T, Watthey L, McDonald L, Artiach P, Bowman C, Garland S, Fuji C, Cotton MD, Horst K, Roberts K, Hatch B, Smith HO, Venter JC. 1997. Genomic sequence of a Lyme disease spirochaete, Borrelia burgdorferi. Nature 390:580586.

80. Kim D, Hong JS, Qiu Y, Nagarajan H, Seo JH, Cho BK, Tsai SF, Palsson BO. 2012.

Comparative analysis of regulatory elements between Escherichia coli and Klebsiella pneumoniae by genome-wide transcription start site profiling. PLoS Genet 8:e1002867. 
81. Prados J, Linder P, Redder P. 2016. TSS-EMOTE, a refined protocol for a more complete and less biased global mapping of transcription start sites in bacterial pathogens. BMC Genomics 17:849.

82. Nowalk AJ, Gilmore RD, Jr., Carroll JA. 2006. Serologic proteome analysis of Borrelia burgdorferi membrane-associated proteins. Infect Immun 74:3864-3873.

83. Livak KJ, Schmittgen TD. 2001. Analysis of relative gene expression data using real-time quantitative PCR and the 2(-Delta Delta C(T)) Method. Methods 25:402-408.

84. Schindelin J, Arganda-Carreras I, Frise E, Kaynig V, Longair M, Pietzsch T, Preibisch S, Rueden C, Saalfeld S, Schmid B, Tinevez JY, White DJ, Hartenstein V, Eliceiri K, Tomancak P, Cardona A. 2012. Fiji: an open-source platform for biological-image analysis. Nat Methods 9:676-682.

85. Takacs CN, Scott M, Chang Y, Kloos ZA, Irnov I, Rosa PA, Liu J, Jacobs-Wagner C. 2020. Code for "A CRISPR interference platform for selective downregulation of gene expression in Borrelia burgdorferi". GitHub Code Repository https://github.com/JacobsWagnerLab/published/tree/master/Takacs_2020_CRISPRi.

86. Mastronarde DN. 2005. Automated electron microscope tomography using robust prediction of specimen movements. J Struct Biol 152:36-51.

87. Zheng SQ, Palovcak E, Armache JP, Verba KA, Cheng Y, Agard DA. 2017. MotionCor2: anisotropic correction of beam-induced motion for improved cryo-electron microscopy. Nat Methods 14:331-332.

88. Kremer JR, Mastronarde DN, McIntosh JR. 1996. Computer visualization of three-dimensional image data using IMOD. J Struct Biol 116:71-76.

89. Narasimhan S, Sukumaran B, Bozdogan U, Thomas V, Liang X, DePonte K, Marcantonio N, Koski RA, Anderson JF, Kantor F, Fikrig E. 2007. A tick antioxidant facilitates the Lyme disease agent's successful migration from the mammalian host to the arthropod vector. Cell Host Microbe 2:7-18. 
90. Morrison TB, Ma Y, Weis JH, Weis JJ. 1999. Rapid and sensitive quantification of Borrelia burgdorferi-infected mouse tissues by continuous fluorescent monitoring of PCR. J Clin Microbiol 37:987-992.

\section{FIGURE LEGENDS}

Figure 1. Summary of relevant genetic constructs. A. Schematic of a mature sgRNA cassette, including its promoter and relevant functional parts. B. Schematic of the inducible dcas9/lacl cassette. C-F. Schematic of shuttle vectors (SV) generated and used in this study. The $B$. burgdorferi and $E$. coli origins of replication and the antibiotic resistance markers are not shown. arr2, rifampin resistance cassette. G. Schematic of the chromosomally encoded dcas9 locus of strain CJW_Bb362. The dcas9/lacl cassette and a kanamycin resistance cassette were inserted in the intergenic region between genes bb0456 and bb0457. ter, transcriptional terminator; aphl, kanamycin resistance gene. $\mathbf{H}$. Mutations introduced into the

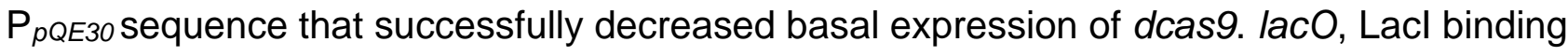
sites; RBS, ribosomal binding site. Mutated residues are shown in red.

Figure 2. Characterization of dcas 9 expression in B. burgdorferi. A. Comparison of dcas 9 mRNA levels measured in the absence of IPTG in control strains that lack an sgRNA. Strain numbers are shown at the bottom. Relevant strain characteristics are marked on the graph. mRNA levels (measured by qRT-PCR) are shown relative to those in strain CJW_Bb430. Chr., chromosomal; SV, shuttle vector. B. Comparison of lac/ mRNA levels measured in the absence of IPTG in the same samples as in panel A. C. Growth curve of strain CJW_Bb242 in the presence of 0 or $0.1 \mathrm{mM}$ IPTG. Cell densities of three replicate cultures were counted daily. Shown are means \pm standard deviations. D. IPTG-mediated induction of dcas 9 expression 
measured by qRT-PCR in the strains indicated below the graph. All values are reported relative to the levels in the uninduced strain CJW_Bb430. Measurements were obtained after one day of induction. E. lacl mRNA levels measured in the same samples as in panel D. A, B, D, and E. Shown are means \pm standard deviations measured from two cultures. A single sample was measured for the induced CJW_Bb430 condition. F. Phase contrast images of cells of the indicated strains after two days of growth in the presence or absence of IPTG.

Figure 3. Effect of CRISPRi on targeted gene mRNA levels. A. flaB, B. mreB, C. rodA, D. ftsl, and E. lacl mRNA levels measured in the indicated control strains (gray) and CRISPRi depletion strains (pink and red) after one day of growth with or without IPTG. Shown are the means \pm standard deviations measured from two cultures. The version of the CRISPRi platform carried by each set of strains is indicated above the corresponding column of graphs. SV, shuttle vector.

Figure 4. Phenotypic characterization of flagellin depletion. A. and B. Inverted darkfield images of strain CJW_Bb242 (A) or CJW_Bb313 (B) grown in the absence or presence of IPTG for two days. The flagellin depletion phenotype is highlighted by a red outline. C. CryoET-based detection of periplasmic flagella in a cell of strain CJW_Bb313 grown in the absence of IPTG. Left: low magnification view of the entire cell. Center: high magnification views of the tip (a) and center (b) of the cell. Right: three-dimensional segmentation of the tip (panel a) and center (panel b) regions of the cell. In panel b, the flagella from one end of the cell are shown in yellow and the flagella from the other end are shown in orange. Also see Fig. S6A. D. Flagellin depletion assessed by cryo-ET in a cell of strain CJW_Bb313 after two days of IPTG exposure. Left: low magnification view of the entire cell. Center: high magnification views of the 
tip (a) and center (b) of the cell. Right: three-dimensional segmentation of the tip (panel a) and center (panel b) of the cell.

Figure 5. Phenotypic characterization of Ftsl depletion. A. Phase contrast micrographs of cells from cultures of strains expressing sgRNAftsl after two days of dcas 9 induction with IPTG or in the absence of IPTG. B. Histograms depicting distributions of cell lengths measured in induced (0.1 mM IPTG for two days, orange) or uninduced (no IPTG, blue) cultures of the noted strains. The strains expressed either no sgRNA (top row) or sgRNAftsl (bottom row).

Figure 6. Phenotypic characterization of MreB and RodA depletion. A and B. Phase contrast micrographs of cells from cultures of CRISPRi strains targeting $\operatorname{mre} B(\mathbf{A})$ or $\operatorname{rod} A(\mathbf{B})$. The marks show the following phenotypes: white arrowheads, cell bulging localized at midcell; blue arrowheads, cell bulging localized at approximately the $1 / 4$ and $3 / 4$ positions along the cell length; yellow arrowheads, cell widening extending along the cell length; pink arrowhead, a widened division site, shown in greater detail in the inset; and white asterisk, cell displaying an enlarged pole. 


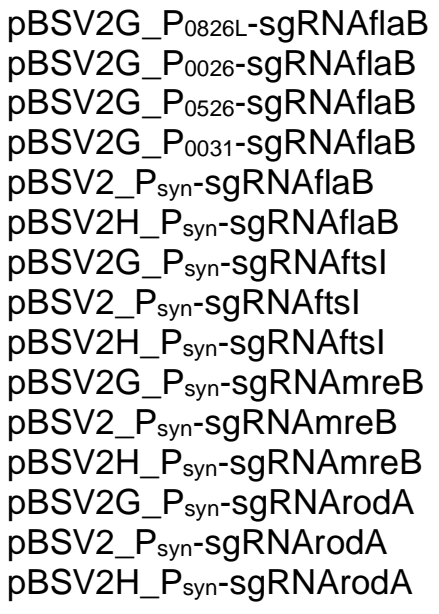

e. dcas 9 shuttle vectors

pBbdCas9Se

pBbdCas9S(RBSmut)

pBbdCas9S(-10TC)

pBbdCas9S_arr2

pBbdCas9S(RBSmut)_arr2

pBbdCas9S(-10TC)_arr2

pBbdCas9K_arr2

pBbdCas9K(RBSmut)_arr2

pBbdCas9K(-10TC)_arr2

pBbdCas9G_arr2

pBbdCas9G(RBSmut)_arr2

pBbdCas9G(-10TC)_arr2

pBbdCas9B_arr2

pBbdCas9B(RBSmut)_arr2

pBbdCas9B(-10TC)_arr2

pBbdCas9H_arr2

pBbdCas9H(RBSmut)_arr2

pBbdCas9H(-10TC)_arr2
$\mathrm{Gm}$

$\mathrm{Gm}$

$\mathrm{Gm}$

$\mathrm{Gm}$

$\mathrm{Km}$

Hy, Rf

$\mathrm{Gm}$

$\mathrm{Km}$

Hy, Rf

$\mathrm{Gm}$

$\mathrm{Km}$

$\mathrm{Hy}, \mathrm{Rf}$

$\mathrm{Gm}$

$\mathrm{Km}$

Hy, Rf

$\mathrm{Sm}, \mathrm{Sp}$

$\mathrm{Sm}, \mathrm{Sp}$

$\mathrm{Sm}, \mathrm{Sp}$

$\mathrm{Sm}, \mathrm{Sp}$

Sm, Sp, Rf

$\mathrm{Sm}, \mathrm{Sp}, \mathrm{Rf}$

$\mathrm{Km}, \mathrm{Rf}$

$\mathrm{Km}, \mathrm{Rf}$

$\mathrm{Km}, \mathrm{Rf}$

$\mathrm{Gm}, \mathrm{Rf}$

$\mathrm{Gm}, \mathrm{Rf}$

$\mathrm{Gm}, \mathrm{Rf}$

Bs, Rf

Bs, Rf

Bs, Rf

Hy, Rf

$\mathrm{Hy}, \mathrm{Rf}$

$\mathrm{Hy}, \mathrm{Rf}$

$\mathrm{Sm}, \mathrm{Sp}$

Sm, Sp

$\mathrm{Sm}, \mathrm{Sp}$

$\mathrm{Sm}, \mathrm{Sp}$
[149627]

[149628]

[149629]

[149630]

[149631]

[149561]

[149562]

[149632]

[149563]

[149566]

[149634]

[149567]

[149568]

[149635]

[149569]

[149638]

[149574]

[149581]

[149639]

[149575]

[149582]

[149570]

[149577]

[149584]

[149571]

[149578]

[149585]

[149572]

[149579]

[149586]

[149573]

[149580]

[149587]

f. All-in-one CRISPRi shuttle vectors carrying a template sgRNA500 cassette

pBbdCas9S_Psyn-SgRNA500

pBbdCas9S_P0526-sgRNA500

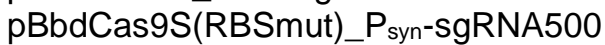

pBbdCas9S(-10TC)_P $P_{\text {syn-SgRNA500 }}$
[149640]

[149641]

[149576]

[149583]

g. All-in-one CRISPRi shuttle vectors carrying mature sgRNA cassettes targeting flaB, ftsl, mreB, or rodA

pBbdCas9S_P syn-sgRNAflaB

pBbdCas9S_PflaBs-sgRNAflaB

pBbdCas9S_Prests-sgRNAflaB

pBbdCas9S_PrestL-sgRNAflaB

pBbdCas9S_P0026-sgRNAflaB

pBbdCas9S_P0826s-sgRNAflaB

pBbdCas9S_P0826L-sgRNAflaB

pBbdCas9S(RBSmut)_P $P_{\text {syn-sgRNAflaB }}$

pBbdCas9S(-10TC)_Psyn-sgRNAflaB

pBbdCas9S_P syn-sgRNAftsI

pBbdCas9S_P0526-sgRNAftsI

pBbdCas9S(RBSmut)_Psyn-sgRNAftsI

pBbdCas9S(-10TC)_Psyn-sgRNAftsI

pBbdCas9S_Psyn-sgRNAmreB

pBbdCas9S_P0526-sgRNAmreB

pBbdCas9S(RBSmut)_P syn-sgRNAmreB
$\mathrm{Sm}, \mathrm{Sp}$

$\mathrm{Sm}, \mathrm{Sp}$

$\mathrm{Sm}, \mathrm{Sp}$

$\mathrm{Sm}, \mathrm{Sp}$

$\mathrm{Sm}, \mathrm{Sp}$

$\mathrm{Sm}, \mathrm{Sp}$

$\mathrm{Sm}, \mathrm{Sp}$

$\mathrm{Sm}, \mathrm{Sp}$

$\mathrm{Sm}, \mathrm{Sp}$

$\mathrm{Sm}, \mathrm{Sp}$

$\mathrm{Sm}, \mathrm{Sp}$

$\mathrm{Sm}, \mathrm{Sp}$

$\mathrm{Sm}, \mathrm{Sp}$

$\mathrm{Sm}, \mathrm{Sp}$

$\mathrm{Sm}, \mathrm{Sp}$

$\mathrm{Sm}, \mathrm{Sp}$
[149642]

[149643]

[149644]

[149645]

[149646]

[149647]

[149648]

[149588]

[149589]

[149649]

[149650]

[149590]

[149591]

[149653]

[149654]

[149594] 
pBbdCas9S(-10TC)_P $P_{\text {syn-sgRNAmreB }}$
pBbdCas9S_P Psyn-sgRNArodA
pBbdCas9S_P0526-sgRNArodA
pBbdCas9S(RBSmut)_Psyn-sgRNArodA
pBbdCas9S(-10TC)_Psyn-sgRNArodA
pBbdCas9S(-10AC1)_Psyn-sgRNArodA
pBbdCas9S(-10AC2)_Psyn-sgRNArodA
pBbdCas9S(-10AC12)_Psyn-sgRNArodA

h. Suicide vector for chromosomal integration of the $d$ cas $9 /$ lac/ cassette pKIKan_idCas9_Chr_center

Sm, Sp
Sm, Sp
Sm, Sp
Sm, Sp
Sm, Sp
Sm, Sp
Sm, Sp
Sm, Sp

$\mathrm{Km}$
[149595]

[149655]

[149658]

[149656]

[149657]

[149658]

[149660]

[149661]

\footnotetext{
${ }^{a}$ This table lists the plasmids used or generated in this study. As indicated, these plasmids are available to the scientific community through Addgene. This includes plasmids that we generated but did not specifically test. For example, for the chromosomal dcas9 variation of the CRISPRi platform, our characterization used the pBSV2Hbased series of sgRNA shuttle vectors. However, we also generated pBSV2- and pBSV2G-based series. We do not expect their behavior to be different from that of the series we tested. We therefore provide these plasmids along with the characterized versions, in the hope that they will be useful to others;

${ }^{b}$ Antibiotic resistance abbreviations: $\mathrm{Cm}$, chloramphenicol; $\mathrm{Gm}$, gentamicin; Km, kanamycin; Sm, streptomycin; $\mathrm{Sp}$, spectinomycin; Bs, blasticidin S; Rf, rifampin; Hy, hygromycin B;

${ }^{c}$ Addgene record numbers are given within brackets for plasmids obtained by us from Addgene, previously generated by us (6) and available from Addgene, or generated by us as part of this study and deposited at Addgene;

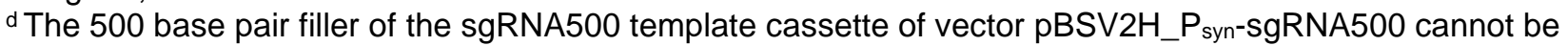
replaced with the base-pairing region of a mature sgRNA by Sapl digestion followed by ligation of annealed primers (Fig. S1A-B) because the hygromycin resistance gene $h p h^{B b}$ also contains a Sapl site. Instead, site-directed mutagenesis, Gibson assembly, or transfer of a mature sgRNA cassette from a different sgRNA shuttle vector can be used to generate a hygromycin B-resistant, mature sgRNA-carrying shuttle vector.

e The name pBbdCas9X stands for inducible expression of dcas9 in Borrelia burgdorferi. The letter $\mathrm{X}$ (with $\mathrm{X}=\mathrm{S}, \mathrm{K}$, $\mathrm{G}, \mathrm{H}$, or $\mathrm{B}$ ) denotes the antibiotic resistance for $B$. burgdorferi selection (streptomycin, kanamycin, gentamicin, hygromycin $\mathrm{B}$, or blasticidin $\mathrm{S}$, respectively).
} 
Table 2. B. burgdorferi strains used or generated in this study

Strain

Genotype / description ${ }^{a}$

Antibiotic

Source or

resistance ${ }^{b}$

reference ${ }^{c}$

\section{Strains previously published}

B31 e2

$\triangle b b e 02::$

$P_{\text {flaB }}$-aadA

B31-A3-68-

$\triangle b b e 02::$

$\mathrm{P}_{\text {flgB }}$-aphl
B31-A3-68-
Reduced genome derivative of the type strain B31

Transformable infectious derivative of the type strain B31.

Genotype: B31-A3-68 Ip25( $\Delta b b e 02:: \mathrm{P}_{\text {fla }}$-aadA) cp9- Ip5- Ip56

Strain routinely referred to as "S9"

Transformable infectious derivative of the type strain B31.

Genotype: B31-A3-68 Ip25( $\Delta$ bbe02:: $P_{\text {flgB }}$-aphI) cp9- Ip5- Ip56

Strain routinely referred to as "K2"
None

Sm

$\mathrm{Km}$

$\mathrm{Km}$

CJW_Bb122 B31 e2 / pBSV2_Psyn-mCherry

b. Chromosomal insertion of the dcas $9 /$ lacl cassette

CJW_Bb362 Genotype: B31-A3-68 Chr[Dnt476219-476250::(PflgB-aphl_P flaB

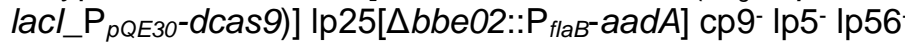

c. Control CRISPRi strains (express no sgRNA cassette)
CJW Bb242
K2 / pBbdCas9S
CJW_Bb410
$\mathrm{K} 2$ / pBbdCas9S(RBSmut)
CJW Bb411
$\mathrm{K} 2$ / pBbdCas9S(-10TC)
CJW_Bb430
CJW_Bb362 / pBSV2H

$\mathrm{Km}, \mathrm{Sm}$

[NR-53512]

$\mathrm{Km}, \mathrm{Sm}$

$\mathrm{Km}, \mathrm{Sm}$

$\mathrm{Km}, \mathrm{Sm}$

$\mathrm{Km}, \mathrm{Sm}, \mathrm{Hy}$

d. flaB CRISPRi strains

CJW_Bb228 K2 / pBbdCas9S_PrestL-sgRNAflaB

CJW_Bb234 K2 / pBbdCas9S_Po826L-sgRNAflaB

CJW Bb235

K2 / pBbdCas9S P0826s-sgRNAflaB

CJW_Bb290

K2 / pBbdCas9S_Prests-sgRNAflaB

CJW Bb312

K2 / pBbdCas9S_PflaBs-sgRNAflaB

CJW Bb313

K2 / pBbdCas9S $P_{\text {syn-sgRNAflaB }}$

K2 / pBbdCas9S Poo26-sgRNAflaB

CJW_Bb314

K2 / pBbdCas9S(RBSmut)_Psyn-sgRNAflaB

CJW_Bb381

K2 / pBbdCas9S(-10TC)_Psyn-sgRNAflaB

CJW-Bb404

CJW_Bb362 / pBSV2H_P ${ }_{\text {syn-sgRNAflaB }}$

$\mathrm{Km}, \mathrm{Sm}$

$\mathrm{Km}, \mathrm{Sm}$

$\mathrm{Km}, \mathrm{Sm}$

$\mathrm{Km}, \mathrm{Sm}$

$\mathrm{Km}, \mathrm{Sm}$

$\mathrm{Km}, \mathrm{Sm}$

$\mathrm{Km}, \mathrm{Sm}$

$\mathrm{Km}, \mathrm{Sm}$

$\mathrm{Km}, \mathrm{Sm}$

$\mathrm{Km}, \mathrm{Sm}, \mathrm{Hy}$

$\mathrm{Km}, \mathrm{Sm}$

$\mathrm{Km}, \mathrm{Sm}$

$\mathrm{Km}, \mathrm{Sm}$

$\mathrm{Km}, \mathrm{Sm}$

$\mathrm{Km}, \mathrm{Sm}, \mathrm{Hy}$

$\mathrm{Km}, \mathrm{Sm}$

$\mathrm{Km}, \mathrm{Sm}$

$\mathrm{Km}, \mathrm{Sm}, \mathrm{Hy}$

$\mathrm{Km}, \mathrm{Sm}$

$\mathrm{Km}, \mathrm{Sm}$

$\mathrm{Km}, \mathrm{Sm}$

$\mathrm{Km}, \mathrm{Sm}$ 

CJW_Bb375
CJW Bb376
CJW Bb380
CJWBBb408

$\mathrm{Km}, \mathrm{Sm}$

$\mathrm{Km}, \mathrm{Sm}$

$\mathrm{Km}, \mathrm{Sm}$

$\mathrm{Km}, \mathrm{Sm}, \mathrm{Hy}$

a For the two B31-A3-68- $\triangle b b e 02$ strains. K2 and S9, and for CJW_Bb362, a detailed genetic description of relevant genetic elements is provided. This includes the nature of the $\triangle$ bbe02 mutation, the insertion of the dcas $9 / \mathrm{lacl}$ cassette into the chromosome (Chr) and a list of the native B31 plasmids missing from these strains;

${ }^{b}$ Antibiotic resistance abbreviations: Km, kanamycin; Sm, streptomycin; Hy, hygromycin B;

c Strain CJW_Bb362 will be deposited with ATCC and will be available from BEI Resources. Its BEI Resources record number is provided in brackets. 
Table 3. Synthetic DNA sequences used for cloning

Name Sequence ${ }^{a}\left(5^{\prime}\right.$ to $\left.3^{\prime}\right)$

\section{Gene block sequence}

AGCTATGACCATGATTACGAATTCGAGCTCGAATTCTAAAGATCTTTGACAGCTAGCTCAGTCCT

MSO AGGTATAATACTAGTGGAAGAGCGAGCTCTTCCGTTTTAGAGCTAGAAATAGCAAGTTAAAATAA GGCTAGTCCGTTATCAACTTGAAAAAGTGGCACCGAGTCGGTGCTTTTTTTGAAGCTTGGGCCC GAACAAAAACTCAAAGCTTGGC ACTGGCCGTCGTTTTACAAC

\section{Primers used for cloning}

MS1

MS2

MS3

MS4

MS5

MS6

MS7

MS8

MS9

MS11

MS12

MS13

MS14

MS15

MS16

NT89

NT90

NT170

NT267

NT268

NT269

NT270

NT350

NT351

NT352

NT353

NT354

NT355

NT374

NT375

NT376

NT377

NT378

NT379

NT382

NT383

NT384

NT385

NT386

NT387

NT388

NT389

NT390

NT391

NT392
TTTTGAAGCTTGGGCCCGAACAAAAACTCAAAGCTTGGCACTGGCCGTCGTTTTACAAC TGAGCTAGCTGTCAAAGATCTTTAGAATTCGAGCTCGAATTCGTAATCATGGTCATAGCT CAGAATTGGAAAGTATTTTATTGCAACACCCAGCTCTTCCGTTTTAGAGCTAGAAATAGC GATCATATTTGTCTATAAGTGTTGACTTTGGCTCTTCCACTAGTATTATACCTAGGAC GTCCTAGGTATAATACTAGTGGAAGAGCCAAAGTCAACACTTATAGACAAATATGATC GCTATTTCTAGCTCTAAAACGGAAGAGCTGGGTGTTGCAATAAAATACTTTCCAATTCTG AGTGAAAATTTAAATTTCTGACTT

AACAAGTCAGAAATTTAAATTTTC

AGTGATATCTATTGCAACAACAGA

ATAGCTAAGCCTATTGAGTATTTCTTATCCATATGTAATTTCTCCTCTTTAATGAATTCT ATTGATTTGAGTCAGCTAGGAGGTGACTAATAAAAGCTTGATCAGATCTCAGCTTTTT AGAATTCATTAAAGAGGAGAAATTACATATGGATAAGAAATACTCAATAGGCTTAGCTAT AAAAAGCTGAGATCTGATCAAGCTTTTATTAGTCACCTCCTAGCTGACTCAAATCAAT GACAGGATGAGGATCGTTTCGCATGATTGCGCCTTCTTGACGAGTTCTTCTGAATTG CAATTCAGAAGAACTCGTCAAGAAGGCGCAATCATGCGAAACGATCCTCATCCTGTC CTCGGTCTATTCTTTTGATTTACATGACCAAAATCCCTTAACG

CGTTAAGGGATTTTGGTCATGTAAATCAAAAGAATAGACCGAG

TATCGGCCGCATGGCTTGTTATGACTG

TATGAGCTCCTAAGTAATGCAAATAATAATCCCACATTG

GACACTAGTCACTATTTTAAATTCCAGGCGATAAAACC

CACCTGCAGCTAGTTTAAAATTTATTTATCTTGATTATTATTTTTTATGGAG

TATCTCGAGCGAATCAAGTATCAACTTCAATTCTTGAG

CTAGTAACCCGGGTAAAAAAACAAAAGATCCTTTAAAGGATCTTTTGTTAATA

CCGGTATTAACAAAAGATCCTTTAAAGGATCTTTTGTTTTTTTACCCGGGTTA

ACTTAAGGGACCTGCAAGTAGTGCAATTTGTTGATGGCCTA

TAGGCCATCAACAAATTGCACTACTTGCAGGTCCCTTAAGT

CCACGGCCGGAATTCTAAAGATCTTTGACAGCTAGCTCAGTCC

TATGGCGCGCCAAAAAAAGCACCGACTCGGTGCC

TATGGATCCGGAAGAGCCAAAGTCAACACTTATAGAC

GTTGTAAAACGACGGCCAGTGCCAAGCTTC

TTAGCTAAAGATTTTAAACTTGGTATAATTGAATTGGAAGAGCCAAAGTCAAC

GTTGACTTTGGCTCTTCCAATTCAATTATACCAAGTTTAAAATCTTTAGCTAA

TTTATTATGCATCCTAGTACATATTATATAATTTAATTTGGAAGAGCCAAAGTCAACAC GTGTTGACTTTGGCTCTTCCAAATTAAATTATATAATATGTACTAGGATGCATAATAAA

TCTTTTTTTTTAATTTTTGTGCTATTCTTTTTAACGGAAGAGCCAAAGTCAACACTT

AAGTGTTGACTTTGGCTCTTCCGTTAAAAAGAATAGCACAAAAATTAAAAAAAAAGA

CTTATATTGACAATCTAAGTATAATATTAAGGGAAGAGCCAAAGTCAACACTT

AAGTGTTGACTTTGGCTCTTCCCTTAATATTATACTTAGATTGTCAATATAAG

GAAAAGTATTTAAATAAGTGTCAATATTTTGTATTATTTAATTGGAAGAGCCAAAGTCAACACTT

AAGTGTTGACTTTGGCTCTTCCAATTAAATAATACAAAATATTGACACTTATTTAAATACTTTTC

TGATATTTTGATTTTTTATGATTAGAATCATCATGGAAGAGCCAAAGTCAACA

TGTTGACTTTGGCTCTTCCATGATGATTCTAATCATAAAAAATCAAAATATCA

CAAAGTTAACAGCAATGAAGTTTATAATAAATTGGAAGAGCCAAAGTCAA

TTGACTTTGGCTCTTCCAATTTATTATAAACTTCATTGCTGTTAACTTTG

AACTAAACTTTGAAAGCCTTGTTATAATATAAAATGGAAGAGCCAAAGTCAAC 
NT393

NT394

NT395

NT402

NT403

NT404

NT405

NT406

NT409

NT410

NT446

NT449

NT593

NT594

NT595

NT596

NT611

NT612

NT619

NT620

NT654

NT655

NT656

NT669

NT670

NT671

NT672

NT673

NT674

NT675

NT676

NT677

NT678

NT680

NT698

NT699

NT700

NT701

NT702

GTTGACTTTGGCTCTTCCATTTTATATTATAACAAGGCTTTCAAAGTTTAGTT

CGAATTCTAAAGATCTTTGACAGCTAGCTCAGTCCTAGGTATAATACTAGTG

GATCCACTAGTATTATACCTAGGACTGAGCTAGCTGTCAAAGATCTTTAGAATTCGAGCT

ATTGAAAATTTAAATTTCTGACTT

TTTGAAAATTTAAATTTCTGACTT

AAGGAAAATTTAAATTTCTGACTT

CATGAAAATTTAAATTTCTGACTT

AATGAAAATTTAAATTTCTGACTT

CTTTTTTTTTAATTTTTGTGCTATTCTTTTTAACGAAAATTTAAATTTCTGACTTGTTTTAGAGCTAG AAATAGCAAGTTAAAATAAGG

CCTTATTTTAACTTGCTATTTCTAGCTCTAAAACAAGTCAGAAATTTAAATTTTCGTTAAAAAGAAT

AGCACAAAAATTAAAAAAAAAG

AATTGGCGCGCCAGGTTAATCTTCAATAACATG

GAGCGGCCGGAAACAGCTATGACATGATTACGAATTCG

CAACAGGGACACGGGCATTATTTACTAGTCACTATTTTAAATTCCAGGCGATAAAACC

TGATTTGAGTCAGCTAGGAGGTGACTAACCCGGGTAAAAAAACAAAAGATCCTTTAAAGG CGCCTGGAATTTAAAATAGTGACTAGTAAATAATGCCCGTGTCCCTGTTGAATAGGG GGATCTTTTGTTTTTTTACCCGGGTTAGTCACCTCCTAGCTGACTCAAATCAATGCG

AGTGAGTGTTATTAAGCATTCTTT

AACAAAGAATGCTTAATAACACTC

AGTGTTAATCTACCTAATATACCA

AACTGGTATATTAGGTAGATTAAC

CATGAGTGTTATTAAGCATTCTTT

CATGATATCTATTGCAACAACAGA

CATGTTAATCTACCTAATATACCA

GATAACAATTTCACACAGAATTCATTAAAGAAGAGAAATTACATATGGATAAGAAATAC

GTATTTCTTATCCATATGTAATTTCTCTTCTTTAATGAATTCTGTGTGAAATTGTTATC

GCTTTGTGAGCGGATAACAATTATACTAGATTCAATTGTGAGCGGATAACAATTTC

GAAATTGTTATCCGCTCACAATTGAATCTAGTATAATTGTTATCCGCTCACAAAGC

GCTTTGTGAGCGGATAACAATTATAATCGATTCAATTGTGAGCGGATAACAATTTCACAC GTGTGAAATTGTTATCCGCTCACAATTGAATCGATTATAATTGTTATCCGCTCACAAAGC GCTTTGTGAGCGGATAACAATTATACTCGATTCAATTGTGAGCGGATAACAATTTCACAC GTGTGAAATTGTTATCCGCTCACAATTGAATCGAGTATAATTGTTATCCGCTCACAAAGC GCTTTGTGAGCGGATAACAATTATAACAGATTCAATTGTGAGCGGATAACAATTTCACAC GTGTGAAATTGTTATCCGCTCACAATTGAATCTGTTATAATTGTTATCCGCTCACAAAGC AACTCTGTTGTTGCAATAGATATC

TATGACGTCATTAGAAAAACTCATCGAGCATCAAATGAAACTGC

TATGACGTCATTAGGTGGCGGTACTTGGGTCGATATCAAAG

TATGACGTCATTAACCTTCCCAAACATAACCACTAGG

TATGACGTCATTATTCTTTAGCTCTAGGTCTAGTACTAGGTCTTCTATTACC

TATGGATCCCAGCTTTTTTTTGAAGTGCCTGGCAGTAAGTTG

\section{Primers used to confirm correct homologous recombination in strain CJW_Bb362}

NT424 GAGTAGTTAAGAGTTCTTCTGAAAG

NT425 CCTATAAAGATATATTGCCTTTAAGTG

NT591 GCGCCAGAGTTGTTTCTGAAACATGGC

NT592 GGTATCGGTCTGCGATTCCGACTCG

NT681 GCGATTGCAGTTGAAGCAGCATGTACTAATGTTCCAGC

NT682 CCCCAACTCTAAGTCCACTTTCAGTAATAGCTCGC

NT683 GGATGGTACTGAGGAATTATTGGTGAAACTAAATCG

NT684 GCTGGTTTTCGCATTCCTTCAGTAACATATTTGACC

a Where applicable, restriction enzyme sites are underlined in the primer sequence. 
Table 4. Primer pairs used to generate the mature sgRNA cassettes ${ }^{a}$

\begin{tabular}{|c|c|c|c|c|c|}
\hline Cassette & Forward primer & Reverse primer & Cassette & Forward primer & Reverse primer \\
\hline $\mathrm{P}_{\text {syn- }}$-sgRNAflaB & MS7 & MS8 & $\mathrm{P}_{0826 L}$-sgRNAflaB & NT402 & MS8 \\
\hline $\mathrm{P}_{s y n}$-sgRNAftsl & NT611 & NT612 & Poo26-sgRNAflaB & NT404 & MS8 \\
\hline $\mathrm{P}_{\text {syn- }}$ sgRNAmreB ${ }^{\mathrm{b}}$ & MS9 & NT680 & Pooz1-sgRNAflaB & NT402 & MS8 \\
\hline $\mathrm{P}_{\text {syn-sgRNArodA }}$ & NT619 & NT620 & $\mathrm{P}_{0526}$-sgRNAflaB & NT405 & MS8 \\
\hline $\mathrm{P}_{\text {rests-sgRNAflaB }}$ & NT403 & MS8 & $\mathrm{P}_{0526}$-sgRNAftsl & NT654 & NT612 \\
\hline $\mathrm{P}_{\text {restL-sgRNAflaB }}$ & NT402 & MS8 & $\mathrm{P}_{0526}$-sgRNAmreB & NT655 & NT680 \\
\hline $\mathrm{P}_{0826 \mathrm{~S} \text {-sgRNAflaB }}$ & NT406 & MS8 & $\mathrm{P}_{0526 \text {-sgRNArodA }}$ & NT656 & NT620 \\
\hline
\end{tabular}

${ }^{a}$ The nucleotide sequences of these primers are provided in Table 3.

${ }^{b}$ While we lost the record of the primer sequences used to generate the $\mathrm{P}_{\text {syn- }}$-sgRNAmreB cassette, primers MS9 and NT680 would allow its regeneration. The sequence of the generated cassette was confirmed to be correct by Sanger DNA sequencing. 
Table 5. Primers used for qRT-PCR

\begin{tabular}{cclc}
\hline Gene & Primer type & Sequence (5' to $\left.3^{\prime}\right)$ & Reference \\
\hline flaB & forward & TTCAATCAGGTAACGGCACA & $(89)$ \\
& reverse & GACGCTTGAGACCCTGAAAG & $(89)$ \\
recA & forward & GTGGATCTATTGTATTAGATGAGGCTCTCG & $(90)$ \\
& reverse & GCCAAAGTTCTGCAACATTAACACCTAAAG & $(90)$ \\
dcas9 9 & forward & AAGTAATGGGGCGGCATAAG & This study \\
& reverse & TCTGGCCCTTTTGAGTTGTC & This study \\
lacl & forward & CCTTGTTGCATTAGGCCATC & This study \\
& reverse & TGTGCCATCCTGCTAATCTC & This study \\
ftsl & forward & CGGAGAAACAAAAGGACTGC & This study \\
& reverse & ATTTGAACCGCTGACACTCC & This study \\
mreB & forward & TGTGATATTGGGGGTGGAAC & This study \\
& reverse & AAATTCGTCACCACCAGTCC & This study \\
rodA & forward & CCACGCTAATTATGTGCCATC & This study \\
& reverse & CCAAAAACCCAAACTCTTCG & This study \\
\hline
\end{tabular}




\section{Figure 1}

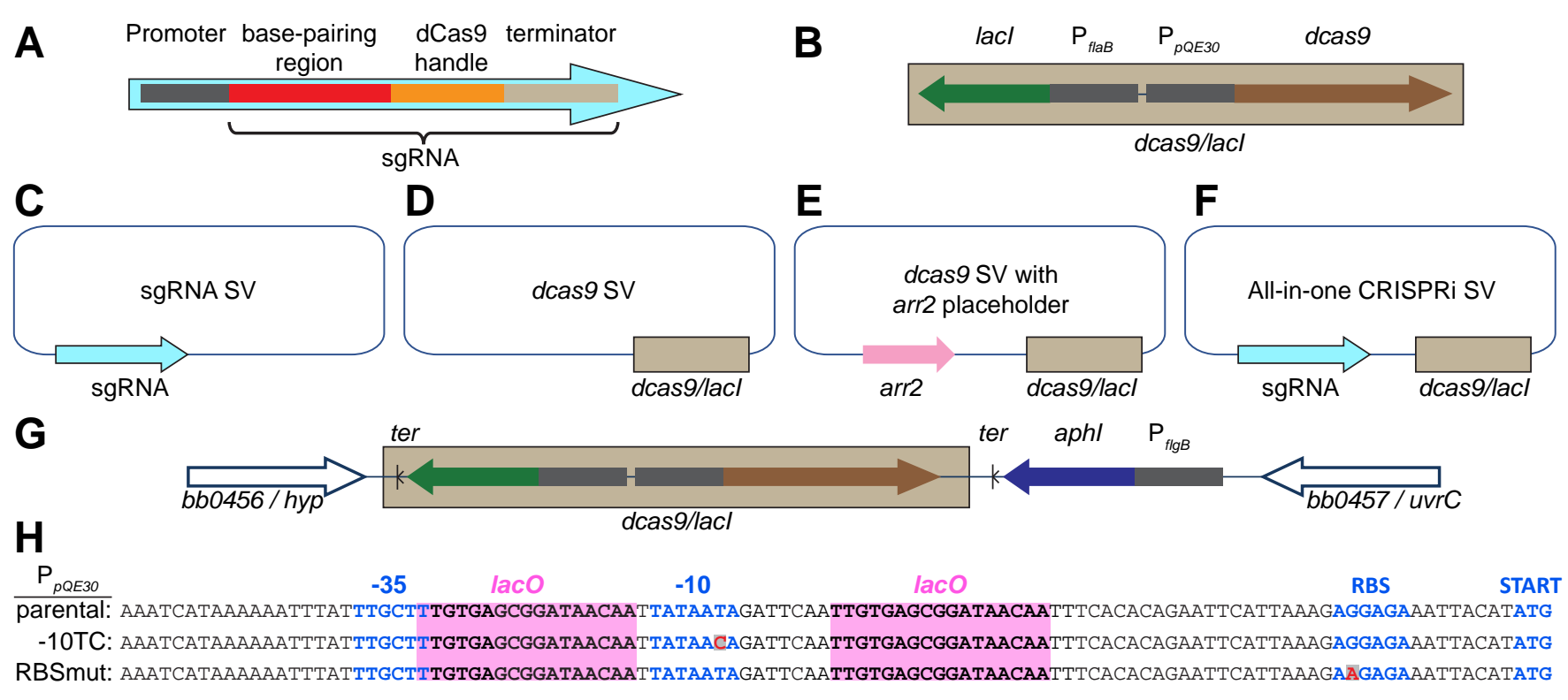

Figure 1. Summary of relevant genetic constructs.

A. Schematic of a mature sgRNA cassette, including its promoter and relevant functional parts.

B. Schematic of the inducible dcas9/lacl cassette.

C-F. Schematic of shuttle vectors (SV) generated and used in this study. The B. burgdorferi and E. coli origins of replication and the antibiotic resistance markers are not shown. arr2, rifampin resistance cassette.

G. Schematic of the chromosomally encoded dcas9 locus of strain CJW_Bb362. The dcas9/lacl cassette and a kanamycin resistance cassette were inserted in the intergenic region between genes bb0456 and bb0457. ter, transcriptional terminator; aphl, kanamycin resistance gene.

H. Mutations introduced into the $\mathrm{P}_{p Q E 30}$ sequence that successfully decreased basal expression of dcas9. lacO, Lacl binding sites; RBS, ribosomal binding site. Mutated residues are shown in red. 


\section{Figure 2}
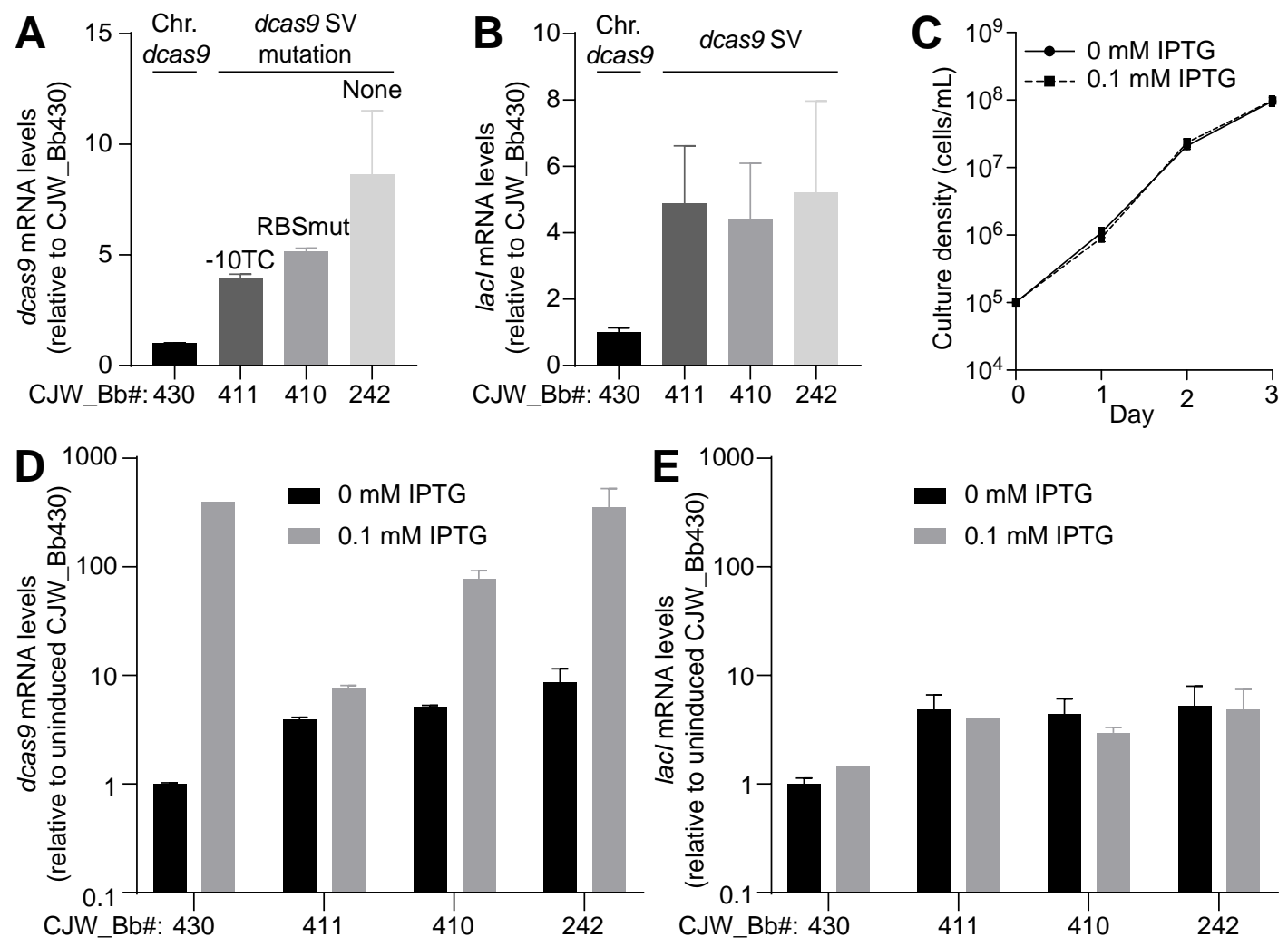

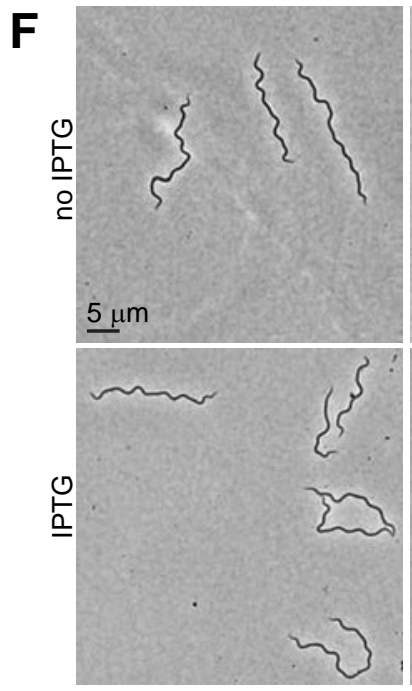

CJW_Bb430

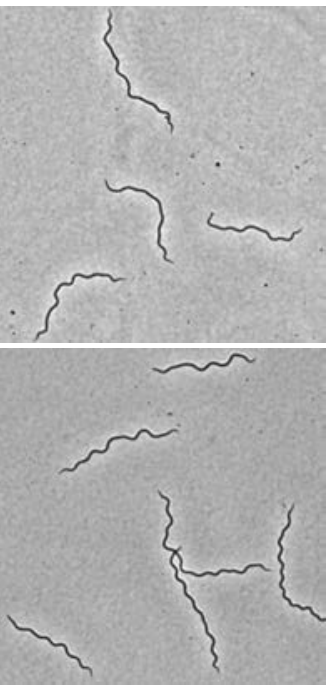

CJW_Bb411

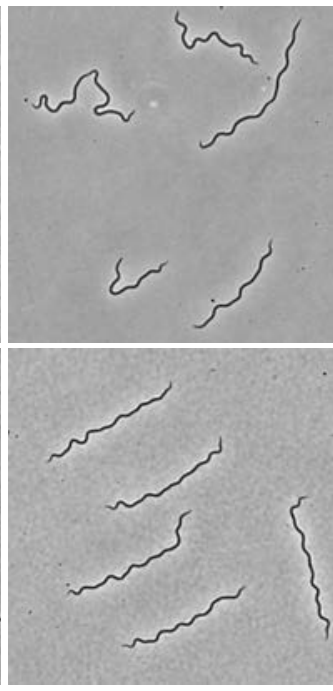

CJW_Bb410

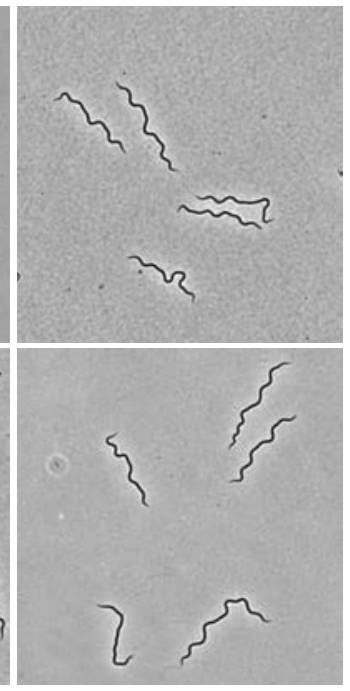

CJW_Bb242

Figure 2. Characterization of dcas9 expression in B. burgdorferi.

A. Comparison of dcas9 mRNA levels measured in the absence of IPTG in control strains that lack an sgRNA. Strain numbers are shown at the bottom. Relevant strain characteristics are marked on the graph. mRNA levels (measured by qRT-PCR) are shown relative to those in strain CJW_Bb430. Chr., chromosomal; SV, shuttle vector.

B. Comparison of lacl mRNA levels measured in the absence of IPTG in the same samples as in panel A.

C. Growth curve of strain CJW_Bb242 in the presence of 0 or $0.1 \mathrm{mM} \mathrm{IPTG.} \mathrm{Cell} \mathrm{densities} \mathrm{of} \mathrm{three} \mathrm{replicate} \mathrm{cultures} \mathrm{were} \mathrm{counted}$ daily. Shown are means \pm standard deviations.

D. IPTG-mediated induction of dcas9 expression measured by qRT-PCR in the strains indicated below the graph. All values are reported relative to the levels in the uninduced strain CJW_Bb430. Measurements were obtained after one day of induction.

E. lacl mRNA levels measured in the same samples as in panel D.

A, B, D, and E. Shown are means \pm standard deviations measured from two cultures. A single sample was measured for the induced CJW_Bb430 condition.

F. Phase contrast images of cells of the indicated strains after two days of growth in the presence or absence of IPTG. 
Figure 3
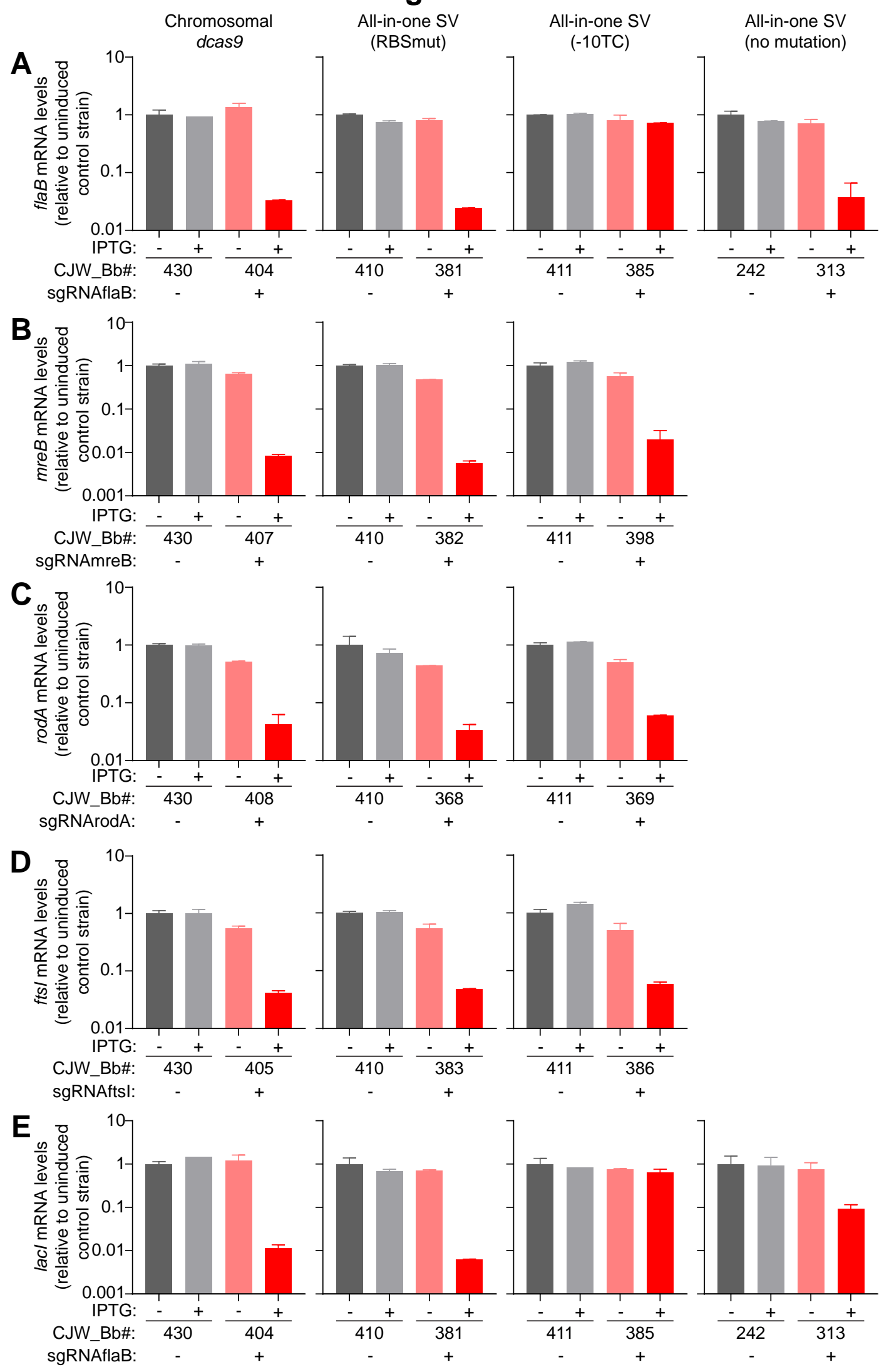

Figure 3. Effect of CRISPRi on targeted gene mRNA levels.

A. $f l a B$, B. $m r e B$, C. $\operatorname{rodA}$, D. ftsl, and E. lacl mRNA levels measured in the indicated control strains (gray) and CRISPRi depletion strains (pink and red) after one day of growth with or without IPTG. Shown are the means \pm standard deviations measured from two cultures. The version of the CRISPRi platform carried by each set of strains is indicated above the corresponding column of graphs. $\mathrm{SV}$, shuttle vector. 


\section{Figure 4}

A

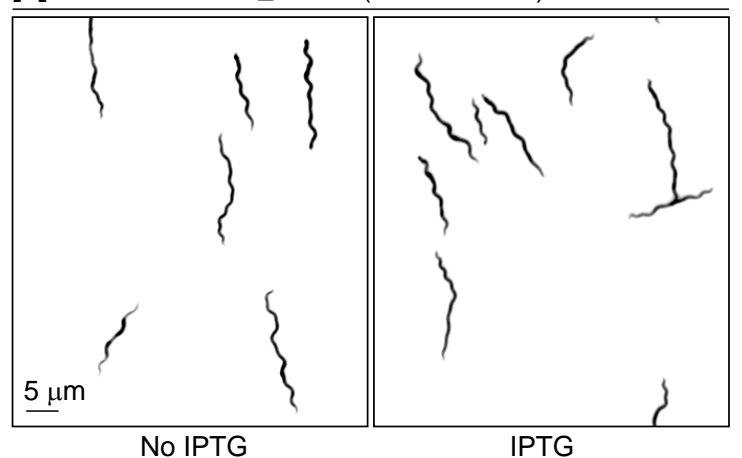

C

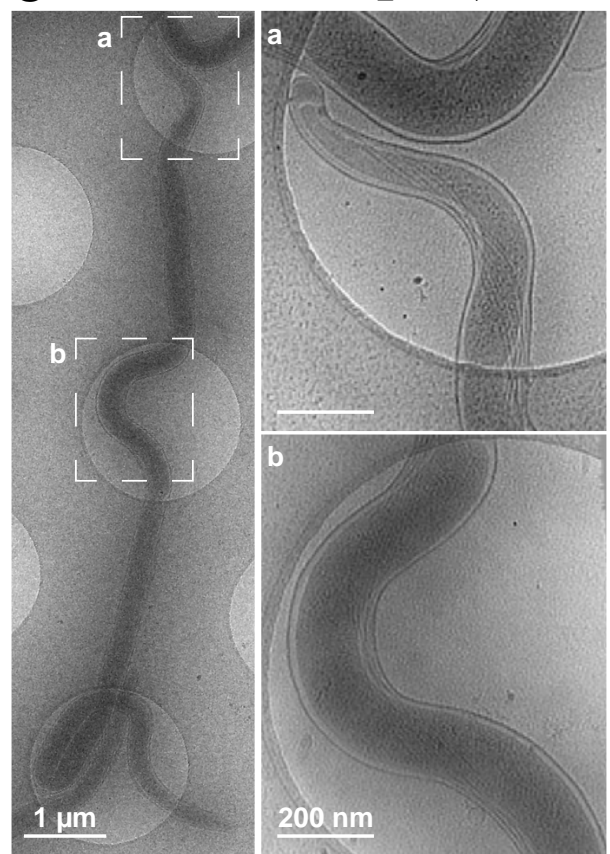

B CJW_Bb313 (FlaB depletion strain)
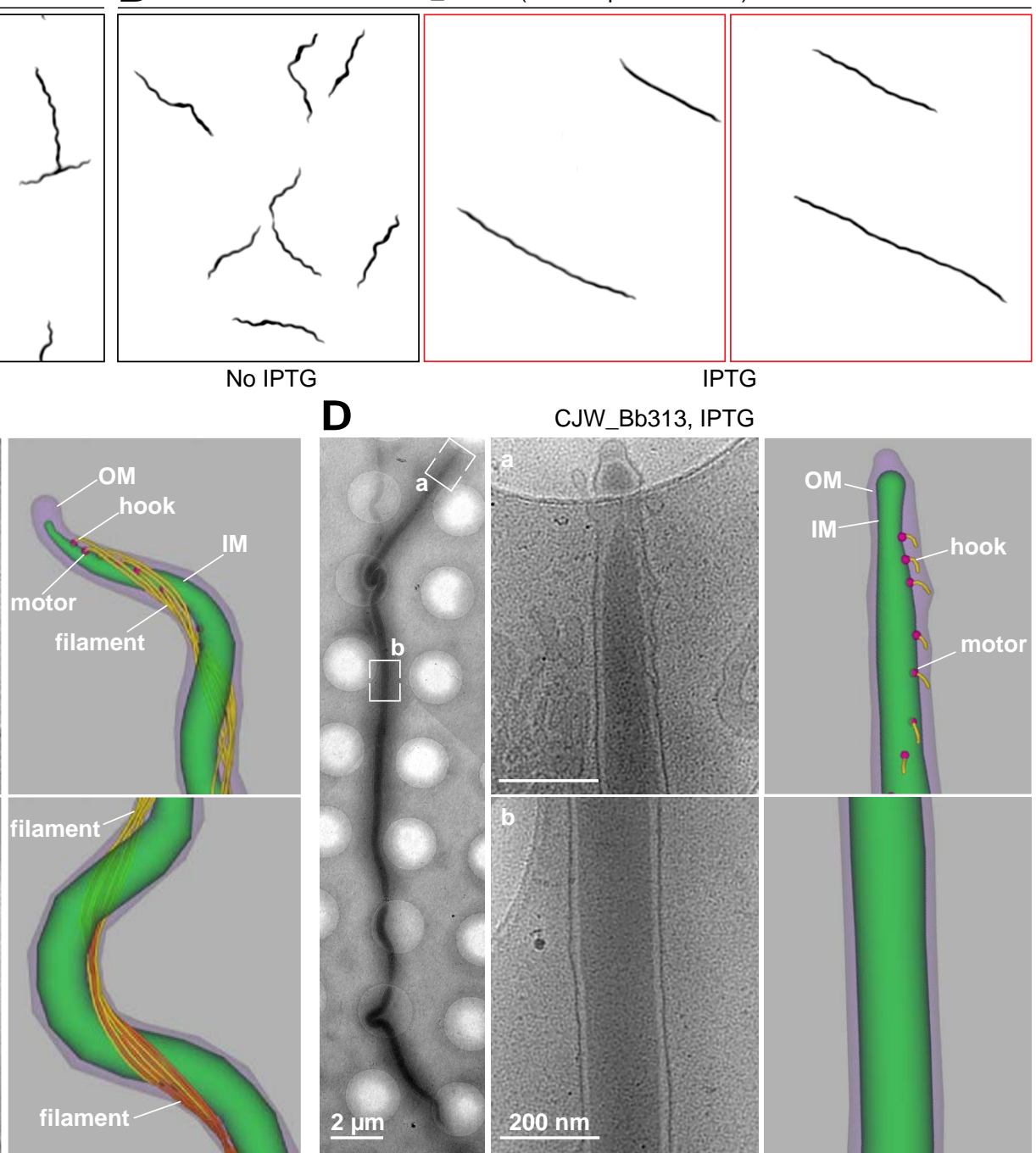

D

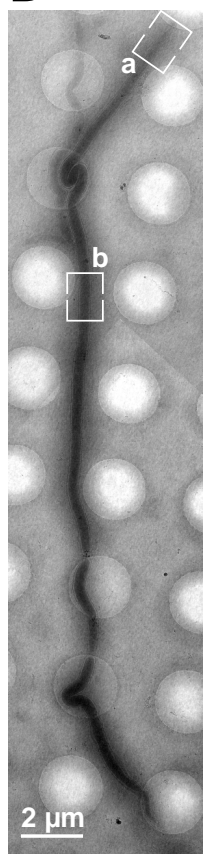

CJW_Bb313, IPTG
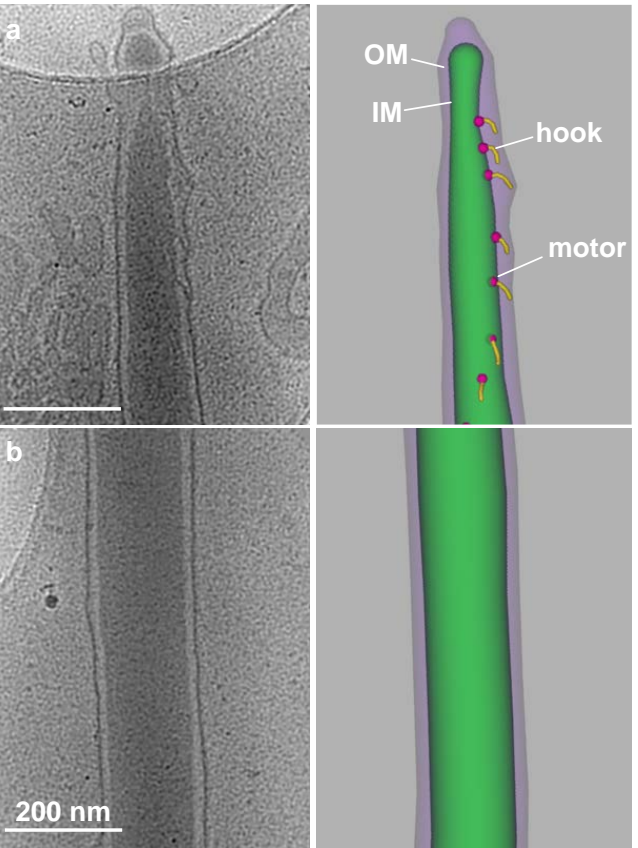

Figure 4. Phenotypic characterization of flagellin depletion.

A. and B. Inverted darkfield images of strain CJW_Bb242 (A) or CJW_Bb313 (B) grown in the absence or presence of IPTG for two days. The flagellin depletion phenotype is highlighted by a red outline.

C. Cryo-ET-based detection of periplasmic flagella in a cell of strain CJW_Bb313 grown in the absence of IPTG. Left: low magnification view of the entire cell. Center: high magnification views of the tip (a) and center (b) of the cell. Right: three-dimensional segmentation of the tip (panel a) and center (panel b) regions of the cell. In panel b, the flagella from one end of the cell are shown in yellow and the flagella from the other end are shown in orange. Also see Fig. S6A.

D. Flagellin depletion assessed by cryo-ET in a cell of strain CJW_Bb313 after two days of IPTG exposure. Left: low magnification view of the entire cell. Center: high magnification views of the tip (a) and center (b) of the cell. Right: three-dimensional segmentation of the tip (panel a) and center (panel b) of the cell. 


\section{Figure 5}

A
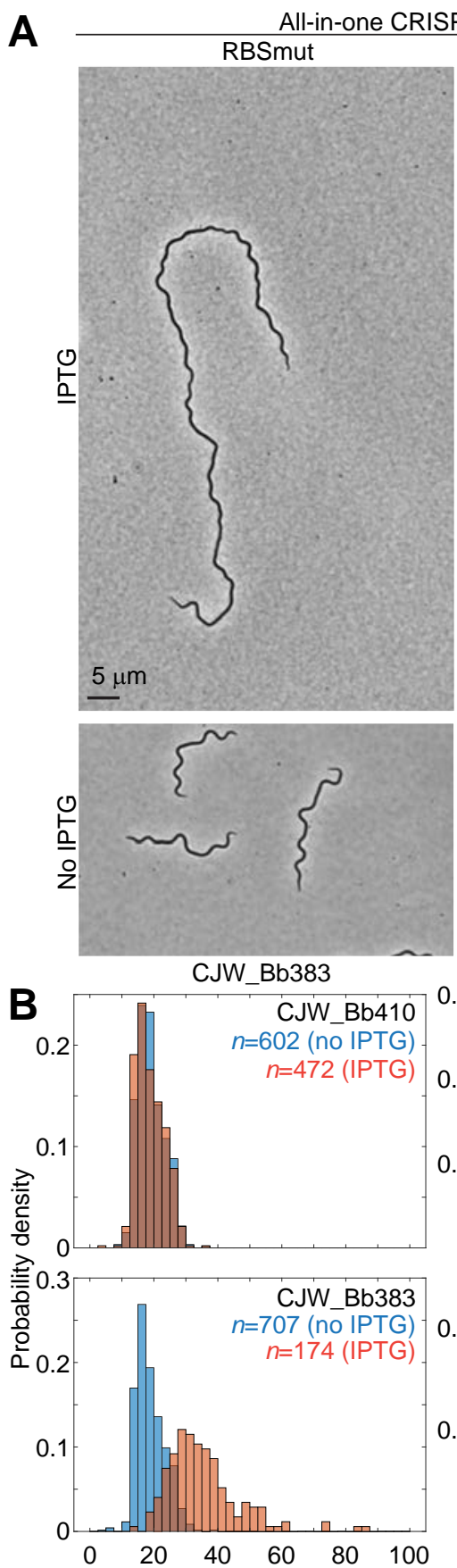

PRi shuttle vector
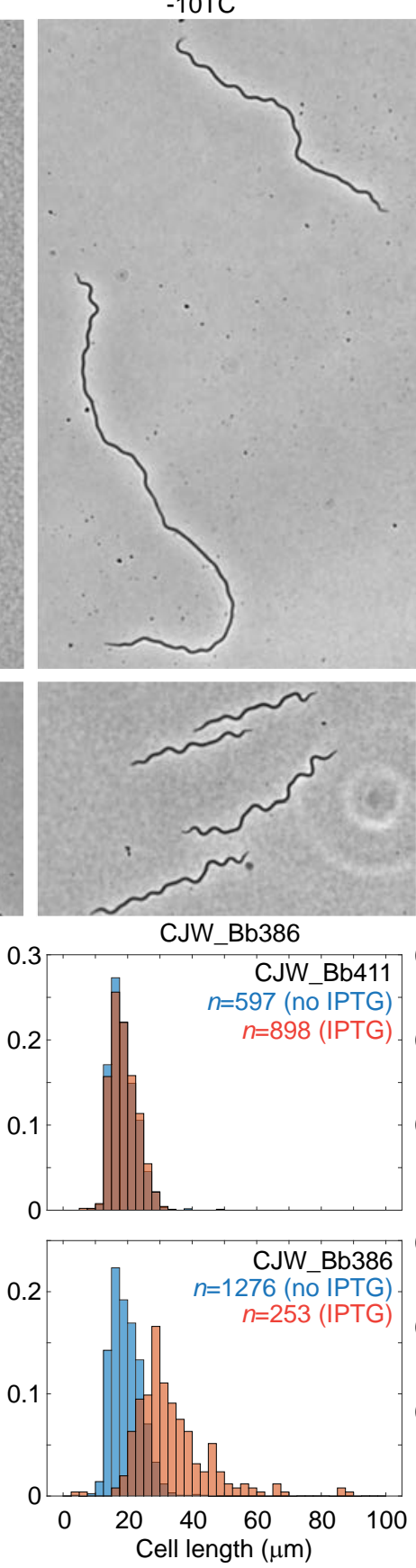
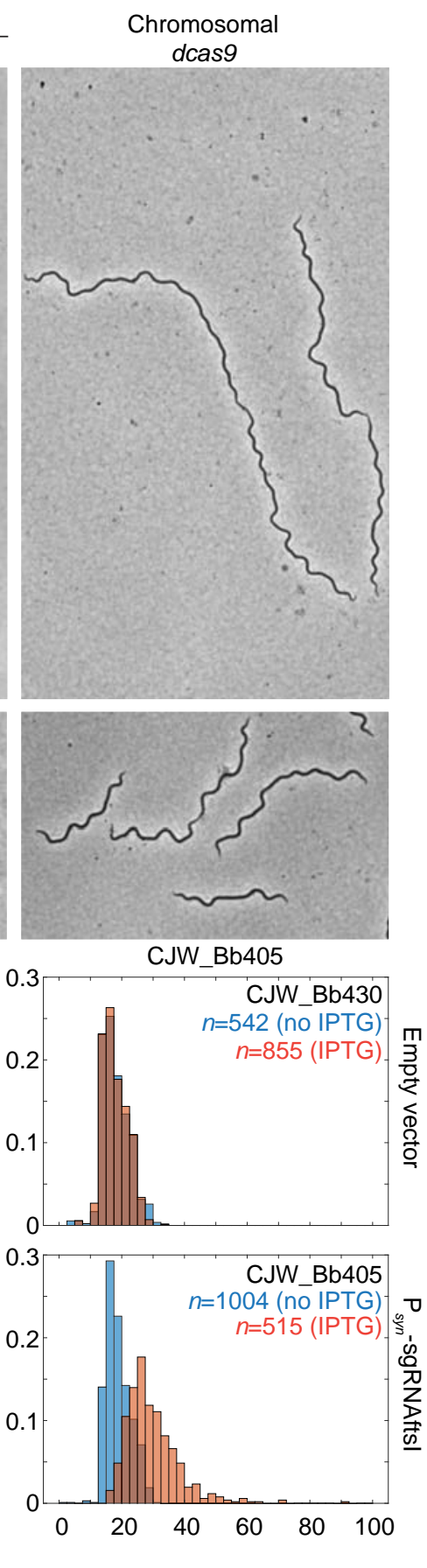

Figure 5. Phenotypic characterization of Ftsl depletion.

A. Phase contrast micrographs of cells from cultures of strains expressing sgRNAftsl after two days of dcas9 induction with IPTG or in the absence of IPTG.

B. Histograms depicting distributions of cell lengths measured in induced (0.1 mM IPTG for two days, orange) or uninduced (no IPTG, blue) cultures of the noted strains. The strains expressed either no sgRNA (top row) or sgRNAftsl (bottom row). 


\section{Figure 6}

A

MreB depletion
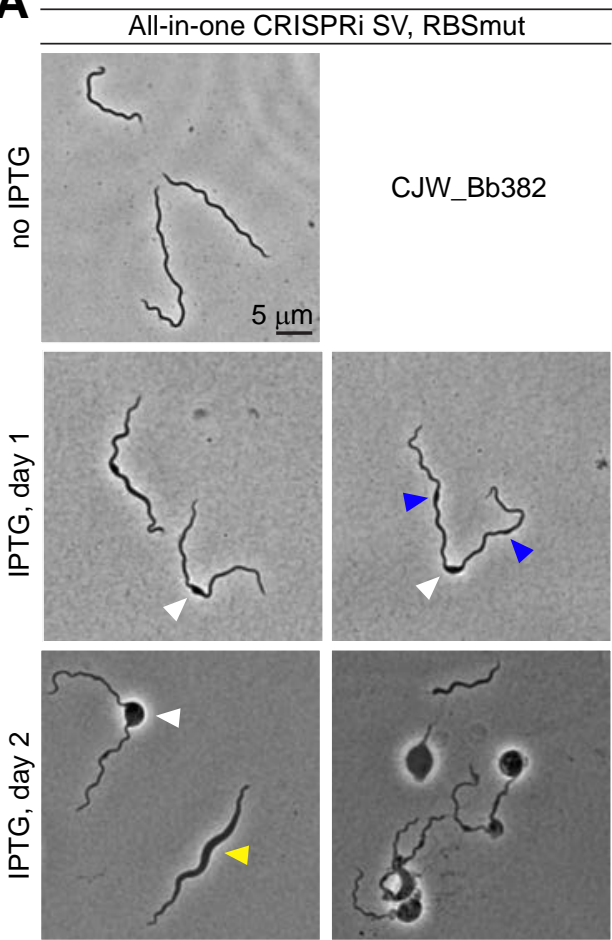

B

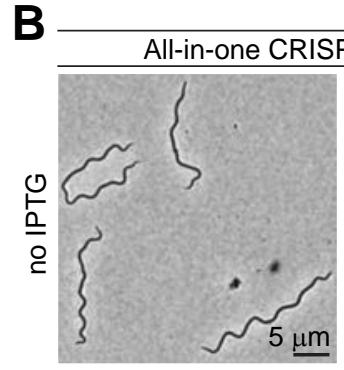

CJW_Bb382

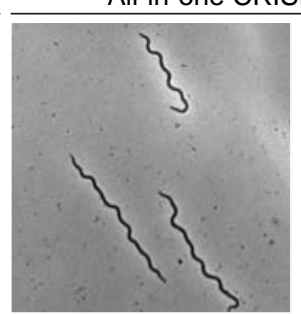

CJW_Bb398
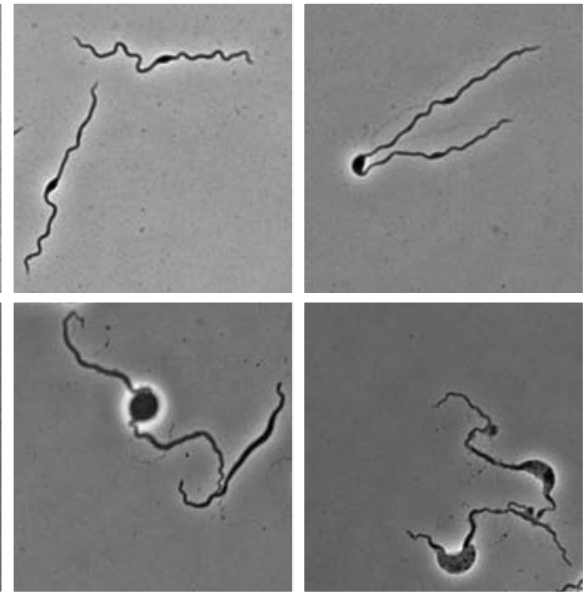

RodA depletion
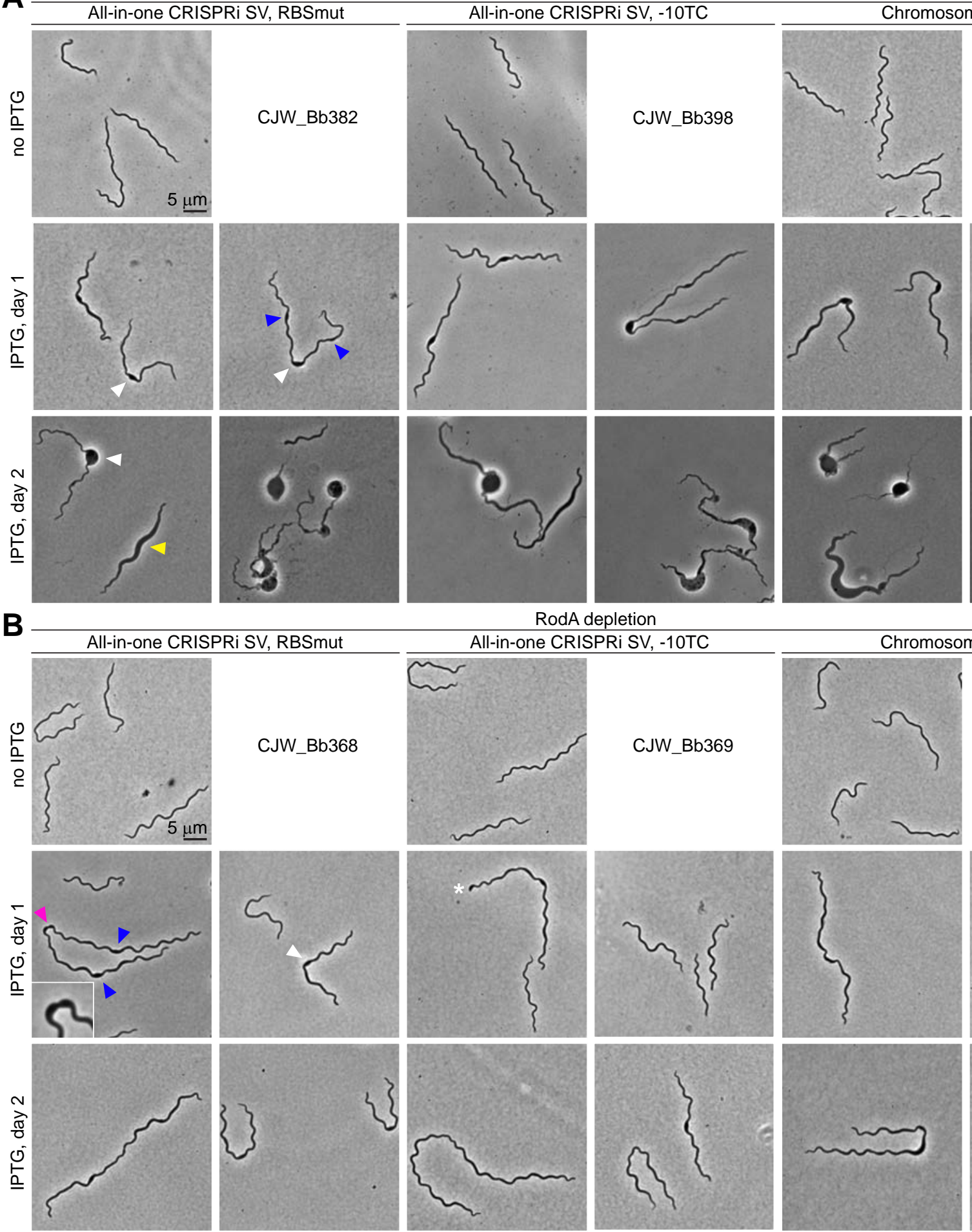

CJW_Bb407
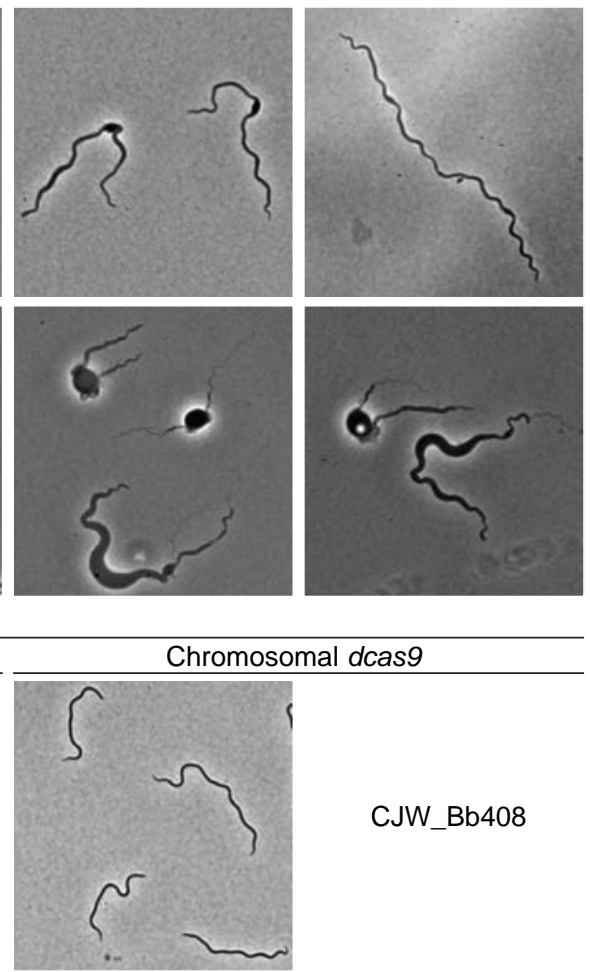

CJW_Bb408
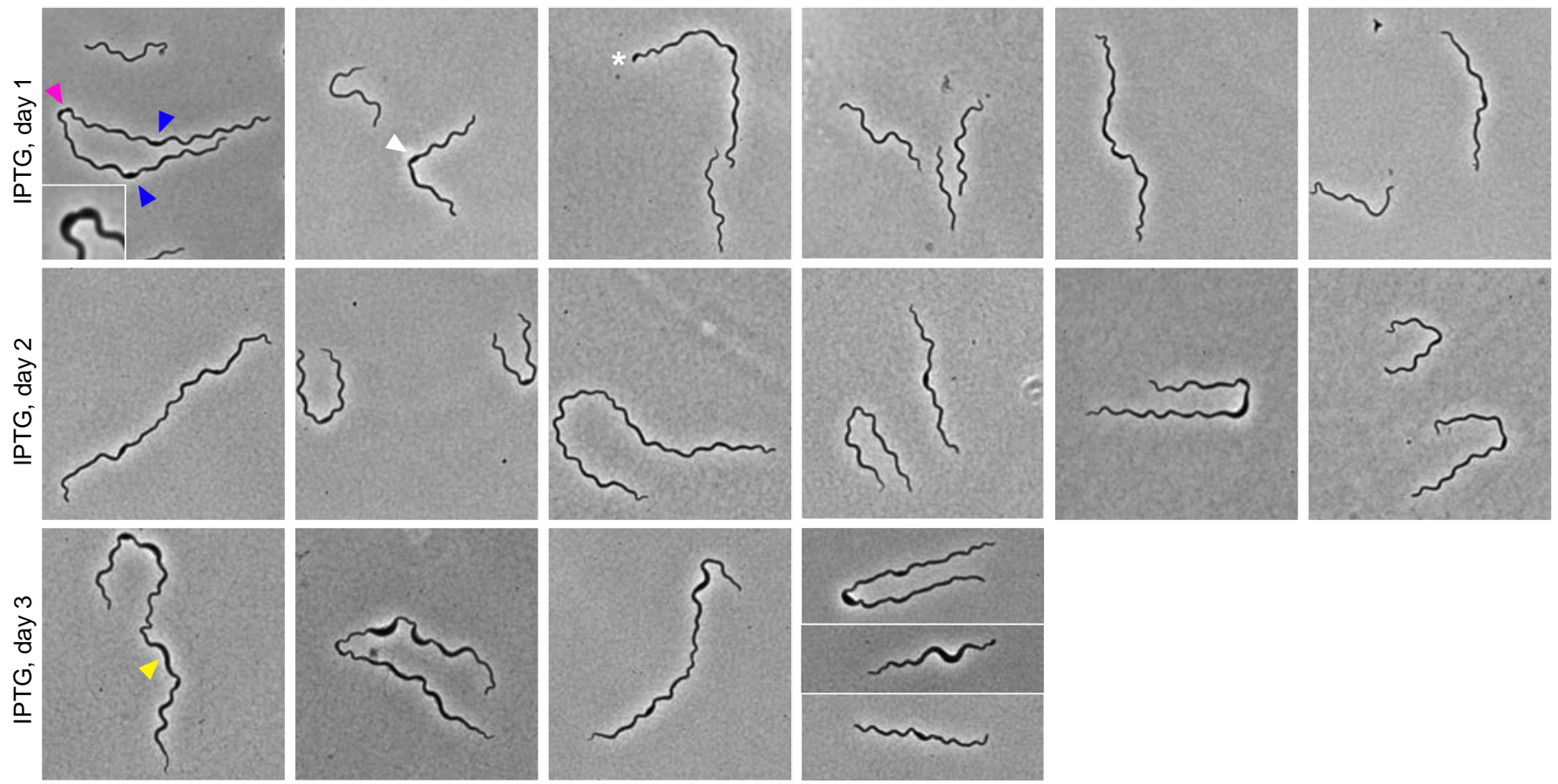

Figure 6. Phenotypic characterization of MreB and RodA depletion.

A and $\mathbf{B}$. Phase contrast micrographs of cells from cultures of CRISPRi strains targeting mre $B(\mathrm{~A})$ or $\operatorname{rod} A(\mathrm{~B})$. The marks show the following phenotypes: white arrowheads, cell bulging localized at midcell; blue arrowheads, cell bulging localized at approximately the $1 / 4$ and $3 / 4$ positions along the cell length; yellow arrowheads, cell widening extending along the cell length; pink arrowhead, a widened division site, shown in greater detail in the inset; and white asterisk, cell displaying an enlarged pole. 


\section{A CRISPR interference platform for selective downregulation of gene expression in Borrelia burgdorferi.}

Constantin N. Takacs, Molly Scott, Yunjie Chang, Zachary A. Kloos, Irnov Irnov, Patricia A. Rosa, Jun Liu, and Christine Jacobs-Wagner

SUPPLEMENTAL MATERIAL:

Supplemental text. S2

Supplemental tables. S3

Supplemental figures. S5

Supplemental movie legends. S12

Supplemental references. S13 


\section{SUPPLEMENTAL TEXT}

\section{Pharmacologic attempts to study MreB function in B. burgdorferi.}

Specific small-molecule inhibitors can be valuable tools in biological investigations. For instance, the compounds A22 and MP265 inhibit the bacterial actin homolog, MreB, causing cell rounding of $E$. coli and other rod-shaped bacteria (1-5). A22 is active in the related spirochete Leptospira biflexa, where it causes cell bulging (6). However, the effects of MreB inhibitors on $B$. burgdorferi morphology have not been reported.

When we exposed B. burgdorferi strain K2 for two days (about seven generations) to MP265 at $50 \mu \mathrm{M}$, the typical dose of MreB inhibitor used in other bacteria $(1,2,6)$, we observed no detectable morphological changes (Fig. S7A). Increasing the MP265 dose to 500 $\mu \mathrm{M}$ or treating $B$. burgdorferi with $500 \mu \mathrm{M}$ A22 also had no apparent effect on cell morphology (Fig. S7A). Both A22 and MP265 were active in BSK-II medium as $50 \mu \mathrm{M}$ of either drug induced cell rounding in BSK-II-grown cells of the E. coli strain MC1000 (Fig. S7B). We also measured the effects of A22 and MP265 on B. burgdorferi growth. The occurrence of cell growth is an important consideration, as cell rounding or bulging phenotypes associated with MreB inactivation are growth-dependent (1). A22 did not affect the growth of $B$. burgdorferi when used at 50 or $500 \mu \mathrm{M}$, nor did MP265 when used at $50 \mu \mathrm{M}$ (Fig. S7C). While $500 \mu \mathrm{M}$ MP265 slightly reduced the growth of our strain (Fig. S7C), a significant amount of growth (about four generations) still occurred during the treatment. Thus, the lack of cell morphology defects in response of A22 or MP265 treatment cannot be attributed to a growth arrest.

Our results therefore indicate that $B$. burgdorferi appears to be resistant to chemical inhibition of MreB by A22 or MP265. 


\section{SUPPLEMENTAL TABLES}

Table S1. Promoters used to drive sgRNA expression in B. burgdorferi

\begin{tabular}{|c|c|c|c|c|c|c|c|}
\hline \multirow{2}{*}{$\begin{array}{l}\text { Promoter } \\
\text { name }\end{array}$} & \multirow{2}{*}{ Native gene } & \multirow{2}{*}{ Strand } & \multirow{2}{*}{$\begin{array}{l}\text { START codon } \\
\text { positiona }^{\mathrm{a}}\end{array}$} & \multirow{2}{*}{$\begin{array}{c}\text { TSS } \\
\text { position }\end{array}$} & \multirow{2}{*}{$\begin{array}{c}\text { Reference } \\
\text { for TSS }\end{array}$} & \multicolumn{2}{|c|}{ Promoter coordinates } \\
\hline & & & & & & 5 ' end & 3 ' end \\
\hline $\mathrm{P}_{\text {syn }}$ & $\begin{array}{c}\text { None (synthetic } \\
\text { promoter) }\end{array}$ & - & - & - & - & - & - \\
\hline $\mathrm{P}_{\mathrm{flaBS}}$ & bb0147 (flaB) & reverse & 148659 & -56 & $(7,8)$ & 149013 & 148716 \\
\hline $\begin{array}{l}\mathrm{P}_{\text {restL }} \\
\mathrm{P}_{\text {rests }}\end{array}$ & $b b b 03$ (resT) & reverse & 2186 & $\begin{array}{l}-3 /-4 \\
-78\end{array}$ & $\begin{array}{c}(8,9) \\
(8)\end{array}$ & $\begin{array}{l}2371 \\
2371\end{array}$ & $\begin{array}{l}2190 \\
2265\end{array}$ \\
\hline Po826L & bb0826 (hyp) & reverse & 870023 & $\begin{array}{c}0 \\
-52 /-54\end{array}$ & $\begin{array}{l}(8) \\
(8)\end{array}$ & $\begin{array}{l}870235 \\
870235\end{array}$ & $\begin{array}{l}870024 \\
870076\end{array}$ \\
\hline $\mathrm{P}_{0526}$ & bb0526 (hyp & forv & 53 & 0 & (8) & 535523 & 535703 \\
\hline $\mathrm{P}_{0031}$ & bb0031 (lepB) & reverse & 29472 & -14 & $(8,9)$ & 29670 & 29487 \\
\hline $\mathrm{P}_{0026}$ & $b b 0026$ (folD2) & reverse & 25623 & -9 & $(8,9)$ & 25752 & 25633 \\
\hline
\end{tabular}

a Nucleotide positions within the chromosome (RefSeq NC_001318.1) or cp26 (for resT, RefSeq NC_001903.1). Records were last accessed on April 30, 2020;

${ }^{b}$ Nucleotide position relative to the first base of the START codon. 
Table S2. Morphology and growth phenotypes observed in the generated CRISPRi strains in the absence of IPTG induction of dcas9 expression.

\begin{tabular}{|c|c|c|c|c|c|c|}
\hline \multirow{2}{*}{$\begin{array}{c}\text { CRISPRi } \\
\text { version }\end{array}$} & \multirow{2}{*}{$\begin{array}{c}\text { Gene } \\
\text { targeted }\end{array}$} & \multirow{2}{*}{$\begin{array}{c}\mathrm{P}_{\text {pQE30 }} \\
\text { mutation }\end{array}$} & \multirow{2}{*}{$\begin{array}{c}\text { sgRNA } \\
\text { promoter }^{b}\end{array}$} & \multirow{2}{*}{$\begin{array}{l}\text { B. burgdorferi } \\
\text { strain number }\end{array}$} & \multicolumn{2}{|c|}{ Phenotype without IPTGc } \\
\hline & & & & & Cell morphology & Culture growth \\
\hline All-in-one & $f l a B$ & None & $\mathrm{P}_{\text {syn }}$ & CJW_Bb313 & $--^{f}$ & - \\
\hline \multirow[t]{23}{*}{ CRISPRi SVd } & & & $P_{f l a B S}$ & CJW_Bb312 & - & - \\
\hline & & & PrestL & CJW_Bb228 & - & - \\
\hline & & & $\mathrm{P}_{\text {rests }}$ & CJW_Bb290 & - & - \\
\hline & & & $\mathrm{P}_{0826 L}$ & CJW_Bb234 & - & - \\
\hline & & & $\mathrm{P}_{0826 \mathrm{~S}}$ & CJW_Bb235 & - & - \\
\hline & & & $\mathrm{P}_{0026}$ & CJW_Bb314 & - & - \\
\hline & & RBSmut & $\mathrm{P}_{\text {syn }}$ & CJW_Bb381 & - & - \\
\hline & & $-10 \mathrm{TC}$ & $\mathrm{P}_{\text {syn }}$ & CJW_Bb385 & - & - \\
\hline & $\operatorname{rod} A$ & None & $\mathrm{P}_{\text {syn }}$ & CJW_Bb346 & Bulges $^{g}$ & Slowi \\
\hline & & & $P_{0526}$ & CJW_Bb380 & Bulges & Slow \\
\hline & & $-10 A C 1$ & $\mathrm{P}_{\text {syn }}$ & CJW_Bb375 & Bulges & Slow \\
\hline & & $-10 A C 2$ & $\mathrm{P}_{s y n}$ & CJW_Bb376 & Bulges & Slow \\
\hline & & $-10 A C 12$ & $\mathrm{P}_{\text {syn }}$ & CJW_Bb367 & Bulges, mild ${ }^{h}$ & Slow \\
\hline & & RBSmut & $\mathrm{P}_{\text {syn }}$ & CJW_BB368 & - & - \\
\hline & & $-10 \mathrm{TC}$ & $\mathrm{P}_{s y n}$ & CJW_Bb369 & - & - \\
\hline & $m r e B$ & None & $\mathrm{P}_{\text {syn }}$ & N.O. ${ }^{e}$ & $\mathrm{~N} / \mathrm{A}$ & $\mathrm{N} / \mathrm{A}$ \\
\hline & & & $\mathrm{P}_{0526}$ & N.O. & $\mathrm{N} / \mathrm{A}$ & $\mathrm{N} / \mathrm{A}$ \\
\hline & & RBSmut & $\mathrm{P}_{\text {syn }}$ & CJW_Bb382 & Bulges, mild & - \\
\hline & & $-10 \mathrm{TC}$ & $P_{s y n}$ & CJW_Bb398 & Bulges, mild & - \\
\hline & $\mathrm{ftsl}$ & None & $\mathrm{P}_{s y n}$ & CJW_Bb351 & Filamentation, mild & Slow \\
\hline & & & $\mathrm{P}_{0526}$ & CJW_Bb363 & Filamentation, mild & Slow \\
\hline & & RBSmut & $\mathrm{P}_{\text {syn }}$ & CJW_Bb383 & - & - \\
\hline & & $-10 \mathrm{TC}$ & $\mathrm{P}_{\text {syn }}$ & CJW_Bb386 & - & - \\
\hline \multirow{4}{*}{$\begin{array}{c}\text { Chromosomal } \\
\text { dcas9 }\end{array}$} & $f l a B$ & None & $\mathrm{P}_{s y n}$ & CJW_BB404 & - & - \\
\hline & $\operatorname{rod} A$ & None & $\mathrm{P}_{\text {syn }}$ & CJW_Bb408 & - & - \\
\hline & mreB & None & $\mathrm{P}_{\text {syn }}$ & CJW_Bb407 & Bulges, mild & - \\
\hline & ftsl & None & $\mathrm{P}_{\text {syn }}$ & CJW_Bb406 & 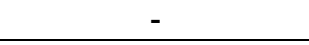 & - \\
\hline
\end{tabular}

a See Fig. $1 \mathrm{H}$ and S1F;

b See Table S1;

c Phenotypes listed reflect our observations during strain generation or clone expansion in liquid medium.

Quantification was not performed;

d SV, shuttle vector;

e N.O., not obtained, likely due to lethality associated with high depletion level of MreB;

${ }^{f}$ No obvious morphology or growth defect observed.

g Cell bulges observed primarily by darkfield microscopy;

${ }^{\mathrm{h}}$ Mild phenotype indicates that only a few cells in the population could be observed to have the noted phenotype; i Slow growth indicates either a prolonged time needed to obtain colonies in semisolid BSK-agarose medium or a slower increase in density of liquid cultures as observed by darkfield imaging. 
A
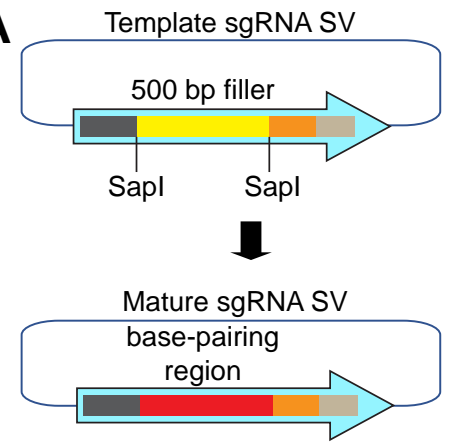

C

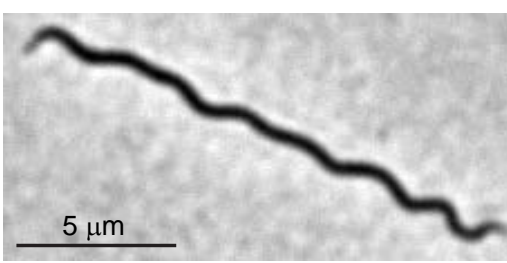

Phase
B

Template sgRNA cassette

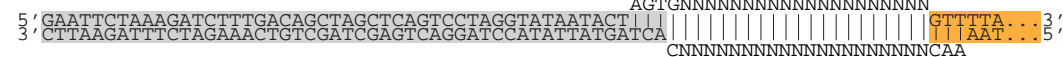

Ligation of annealed primer pair

Mature sgRNA cassette

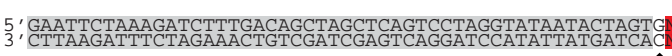

TSS $\longrightarrow$ base-pairing region
dCas9 handle + terminator

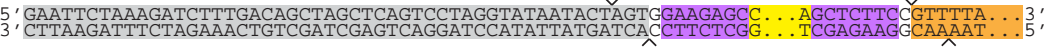

Sapl digestion

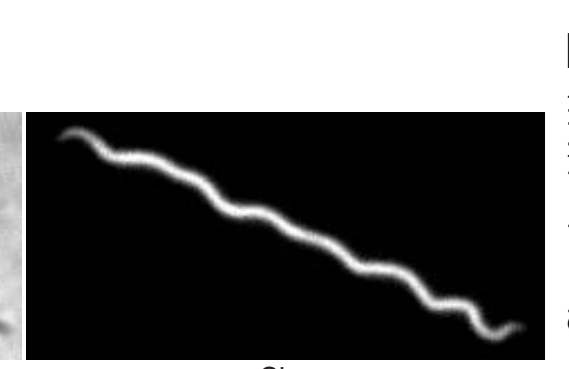

mCherry

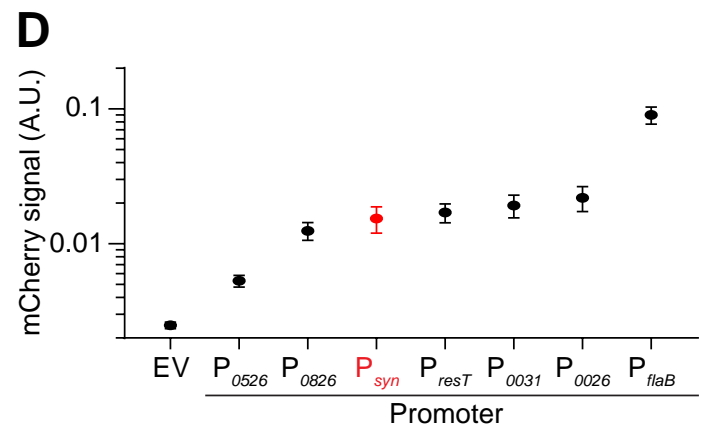

$\mathbf{E}$

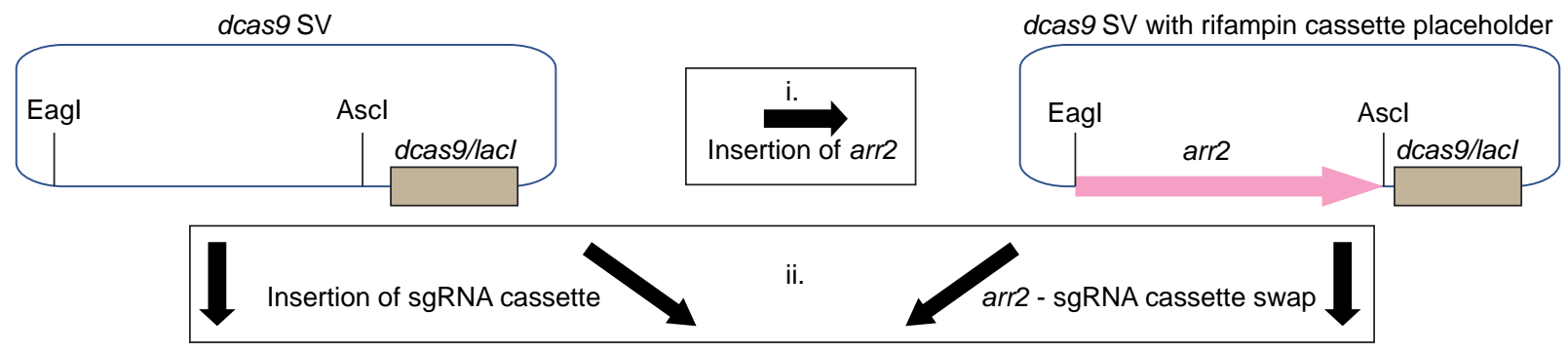

All-in-one CRISPRi SV with template sgRNA cassette
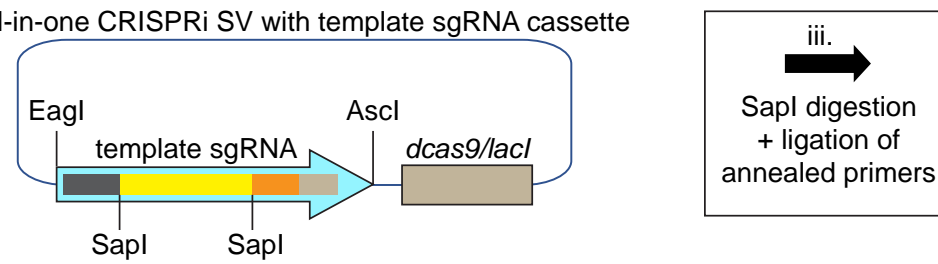

All-in-one CRISPRi SV with mature sgRNA cassette

$\mathbf{F}$ $P_{p Q E 30}$ $-35$ IacO $-10$ lacO

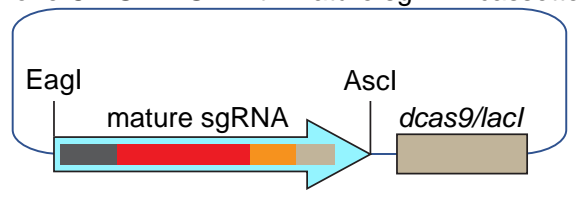

parental: AAATCATAAAAAATTTATTTGCTTTGTGAGCGGATAACAATTATAATAGATTCAATTGTGAGCGGATAACAATTTCACACAGAATTCATTAAAGAGGAGAAATTACATATG -10AC1: AAATCATAAAAAATTTATTTGCTTTGTGAGCGGATAACAATTATACTAGATTCAATTGTGAGCGGATAACAATTTCACACAGAATTCATTAAAGAGGAGAAATTACATATG -10AC2: AAATCATAAAAAATTTATTTGCTTTGTGAGCGGATAACAATTATAATCGATTCAATTGTGAGCGGATAACAATTTCACACAGAATTCATTAAAGAGGAGAAATTACATATG -10AC12: AAATCATAAAAAATTTATTTGCTTTGTGAGCGGATAACAATTATACTCGATTCAATTGTGAGCGGATAACAATTTCACACAGAATTCATTAAAGAGGAGAAATTACATATG

Figure S1. CRISPRi platform construction details.

A. Schematic of conversion of a template sgRNA shuttle vector (SV, top) into a mature sgRNA cassette shuttle vector (bottom).

B. Detail of the process outlined in A. The promoter shown is $P_{\text {syn }}$. The 500 base-pair (bp) DNA filler is released by Sapl digestion. Annealed primers are then ligated to the resulting backbone, generating the mature sgRNA's base-pairing region placed immediately downstream of the promoter's transcriptional start site (TSS).

C. Phase-contrast and fluorescence images of a cell of strain CJW_Bb122 expressing mCherry from $P_{\text {syn }}$.

D. Quantification of the $P_{\text {syn }}$ strength through cellular mCherry fluorescence intensity measurements. The data for all other promoters was obtained and presented in reference (10). A.U., arbitrary units; EV, empty vector.

E. Cloning avenues for generation of all-in-one CRISPRi shuttle vectors. Top left: $d c a s 9$ shuttle vector. Insertion (cloning path i.) of a rifampin resistance cassette, arr2, between its Ascl and Eagl sites generates a dcas9 shuttle vector with a rifampin cassette placeholder (top right). Insertion of a sgRNA cassette between the same sites of the dcas9 shuttle vector (cloning path ii.) yields all-in-one shuttle vectors carrying either a template sgRNA cassette (bottom left) or a mature sgRNA cassette (bottom right). Using the dcas9 shuttle vector with a rifampin cassette (top right) as a starting point in this cloning step offers the advantage of easy screening of clones by checking for loss or rifampin resistance. Conversion of the template sgRNA cassette of the all-in-one CRISPRi shuttle vector (bottom left) into the mature sgRNA cassette (bottom right) requires Sapl digestion followed by ligation of annealed primers (cloning path iii.), as outlined in panel B.

F. Mutations, other than those shown in Fig. $1 \mathrm{H}$, introduced into the $\mathrm{P}_{P Q E 30}$ sequence in an attempt to decrease basal expression of dcas9. lacO, Lacl binding sites; RBS, ribosome-binding site. 


\section{Figure S2}

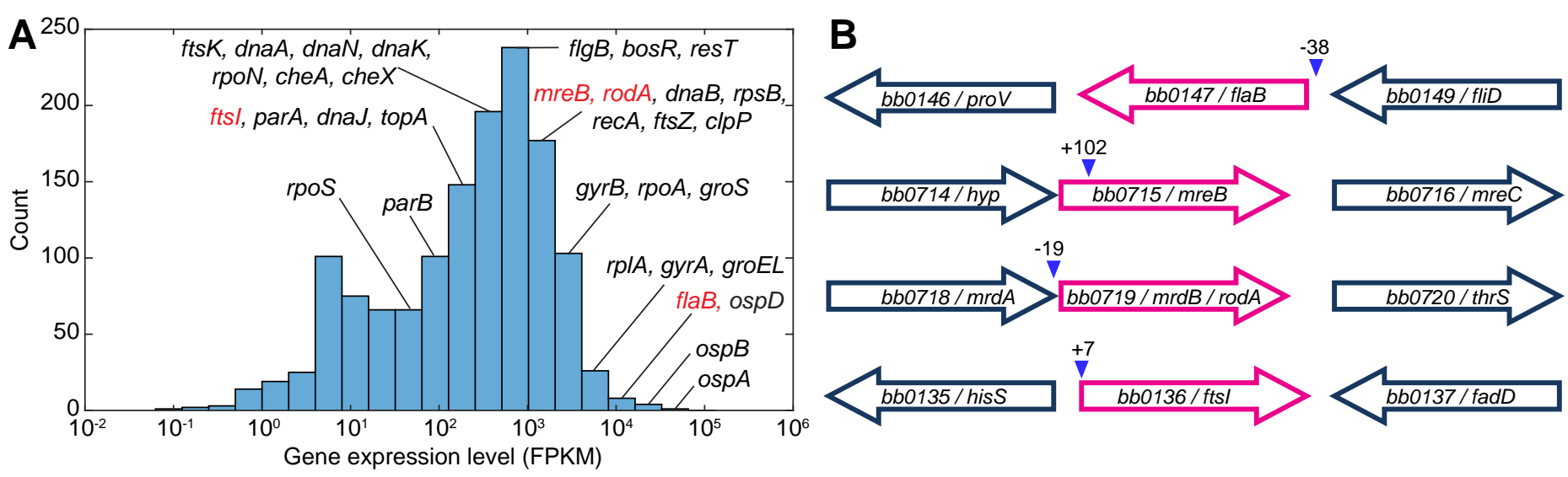

Figure S2. Genes targeted by CRISPRi in B. burgdorferi.

A. Histogram showing the distribution of B. burgdorferi gene expression levels. The RNAseq data was obtained and reported by Arnold el al. (9) using strain B31-A3 (11) grown in BSK-II liquid culture. The data shown is from the early exponential condition as described in the original publication. Each bin represents a 2 -fold range of expression levels. Indicated are expression bins where many physiologically important genes are located. Highlighted in red are the genes targeted by CRISPRi in our study. FPKM, fragments per kilobase per million reads.

B. Genomic context of genes targeted by CRISPRi and location of CRISPR target sites. Coding regions of targeted genes are shown in pink, while upstream and downstream genes are in dark blue. When the distance between two adjacent genes is so short as to suggest that the genes form an operon, the genes are drawn close to each other. However, previous studies (7-9) have identified transcriptional start sites immediately upstream of the START codons of each of these genes. Labels contain both the gene number and its abbreviation. Blue arrowheads show the location of the CRISPR target sites, always within the 5' UTR or the coding sequence of the gene. Values indicate the nucleotide position relative to the translational start site where the 5 ' foremost complementary base of the sgRNA binds. Arrows are not drawn to scale. 


\section{Figure S3}

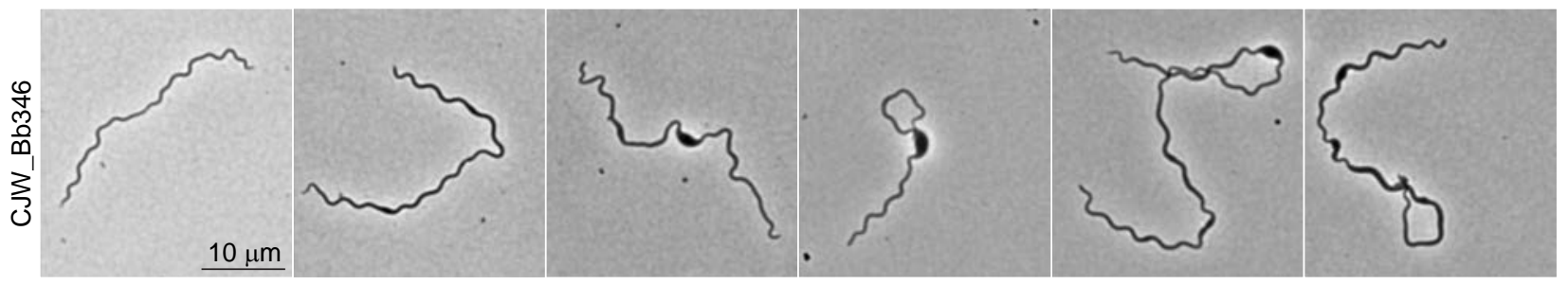

Figure S3. RodA depletion phenotype in the absence of IPTG induction.

Phase contrast images of cells of strain CJW_Bb346 grown without IPTG. A cell with a normal width is at the left, while the other panels show various degrees of cell bulging. 


\section{Figure S4}
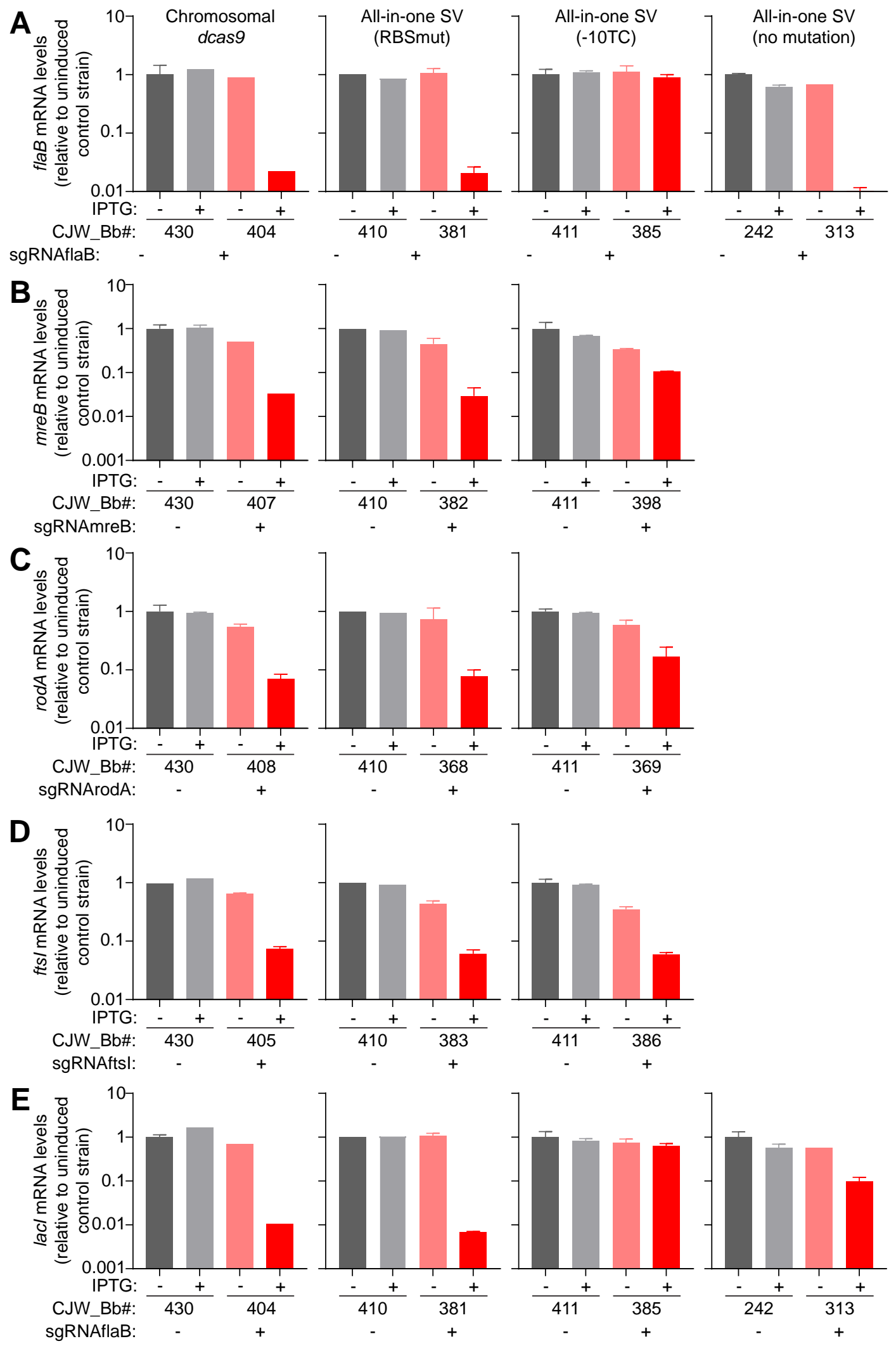

Figure S4. Effect of CRISPRi on targeted gene mRNA levels.

A. flaB, B. mreB, C. rodA, D. ftsl, and E. lacl mRNA levels measured in the indicated control strains (gray) and CRISPRi depletion strains (pink and red) after two days of growth with or without IPTG. Shown are the means \pm standard deviations measured from two cultures, or the values of single measurements (when no error bar is present). The version of the CRISPRi platform carried by each set of strains is indicated above the corresponding column of graphs. SV, shuttle vector. 


\section{Figure S5}
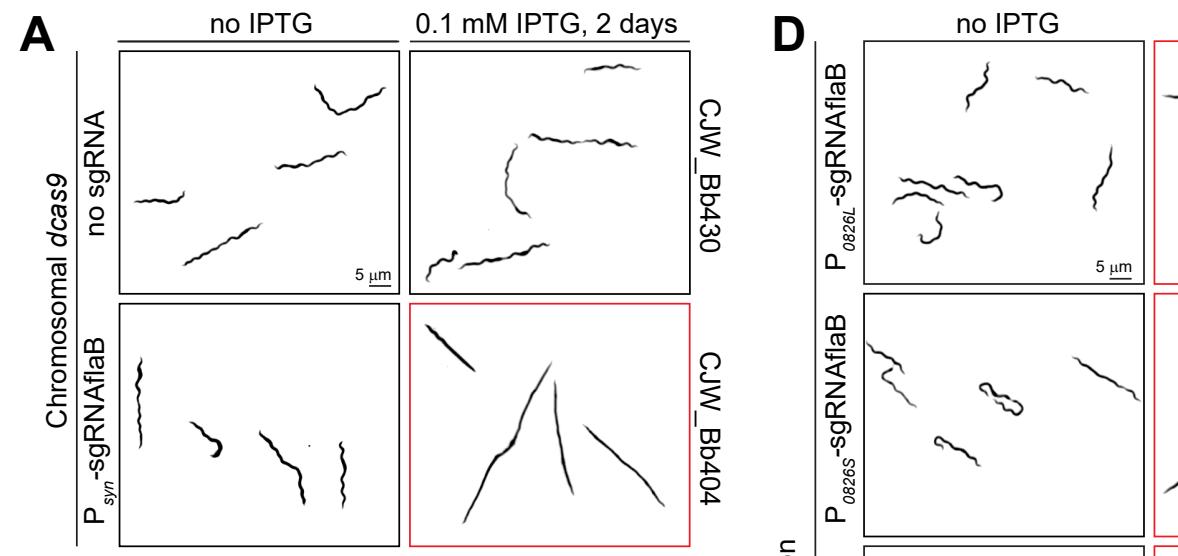

$0.1 \mathrm{mM}$ IPTG, 2 days

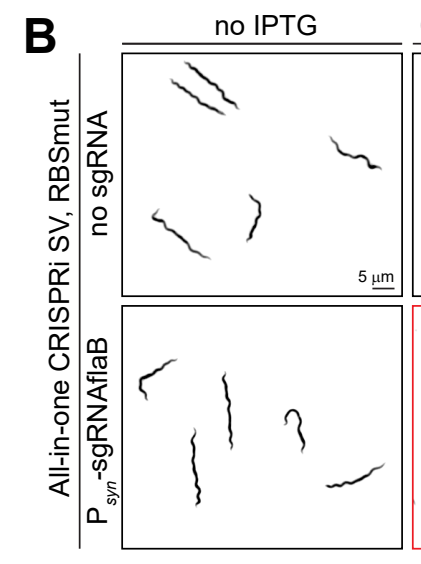

$0.1 \mathrm{mM}$ IPTG, 2 days

C

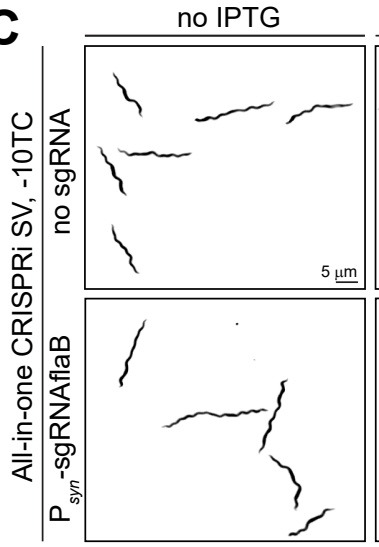

$0.1 \mathrm{mM}$ IPTG, 2 days

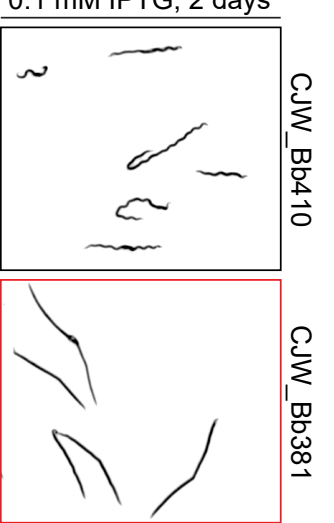

Figure S5. Darkfield microscopy characterization of flagellin depletion strains.

A-C. Inverted darkfield images of strains expressing either no sgRNA or sgRNAflaB from versions of the CRISPRi platform that reduce the basal expression of dcas9.

D. Inverted darkfield images of strains carrying flaB-targeting all-in-one CRISPRi shuttle vectors. The strains differ only in the promoter used to drive sgRNAflaB expression, as noted.

A-D. Images showing a flagellin depletion phenotype are outlined in red. SV, shuttle vector. 


\section{Figure S6}

A

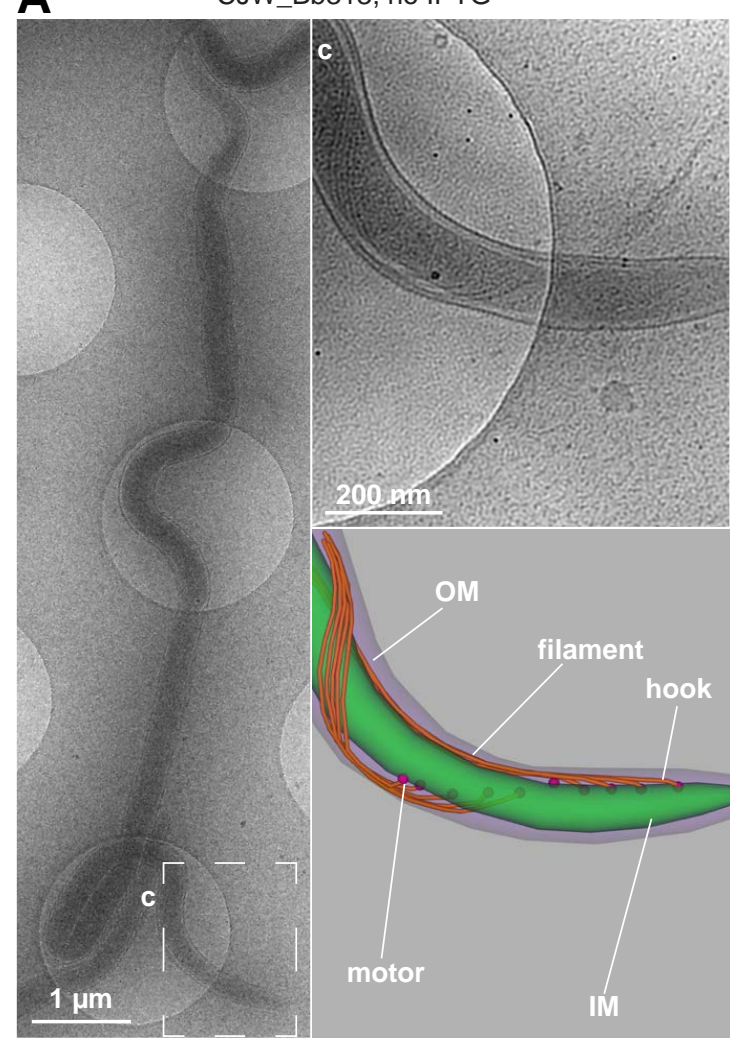

B

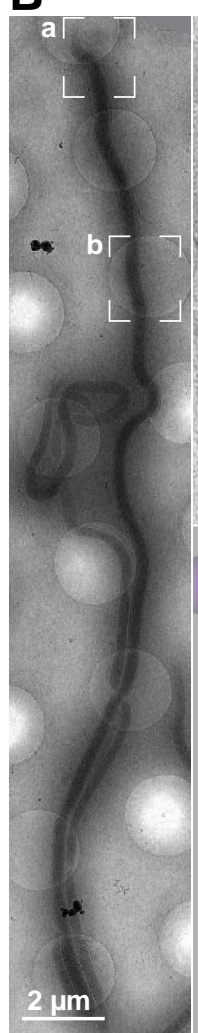

CJW Bb313, IPTG

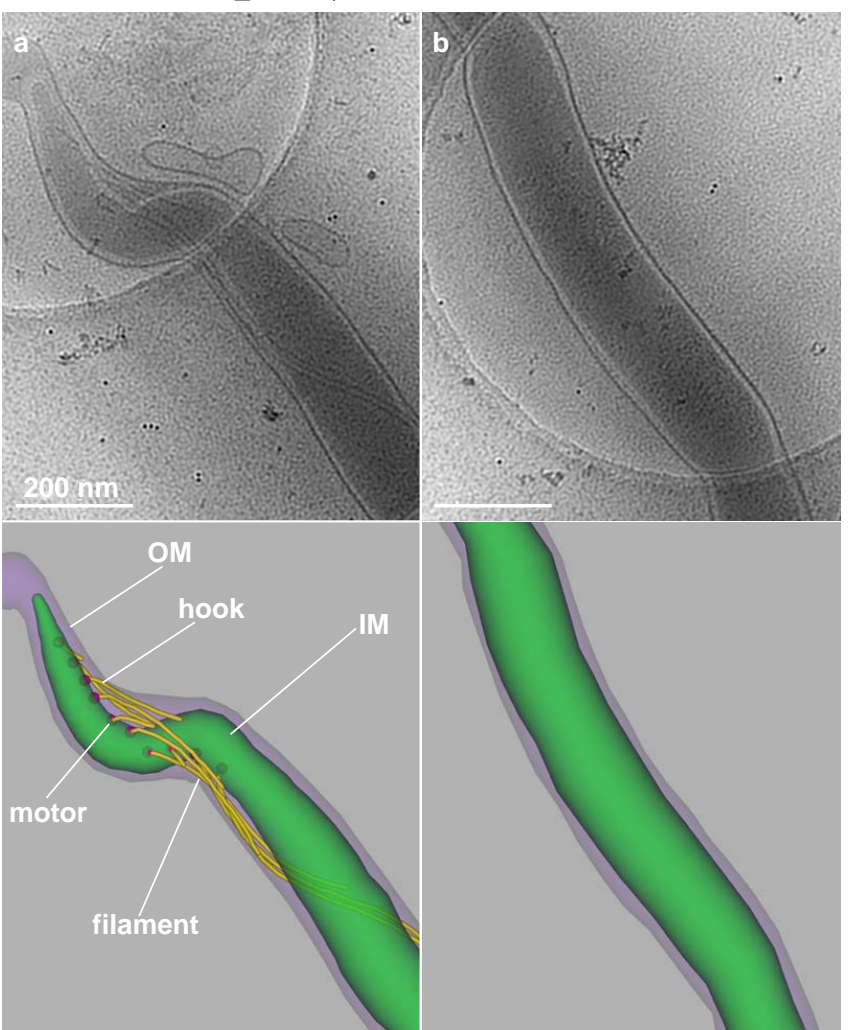

Figure S6. Cryo-ET characterization of flagellin depletion.

A. Cryo-ET-based detection of periplasmic flagella in a cell of strain CJW_Bb313 grown in the absence of IPTG. Shown at the left is a low magnification view of the same cell as in Fig. 4C. Top right: high magnification view of the bottom end (c) of the cell. Bottom right: threedimensional segmentation of the bottom end region of the cell.

B. Flagellin depletion assessed by cryo-ET in a cell of strain CJW_Bb313 after two days of IPTG exposure. Left: low magnification view of the entire cell. Top center and right: high magnification views of the end (a) and center (b) of the cell, respectively. Bottom center and right: threedimensional segmentation of the end and center of the cell, respectively. 
Figure S7
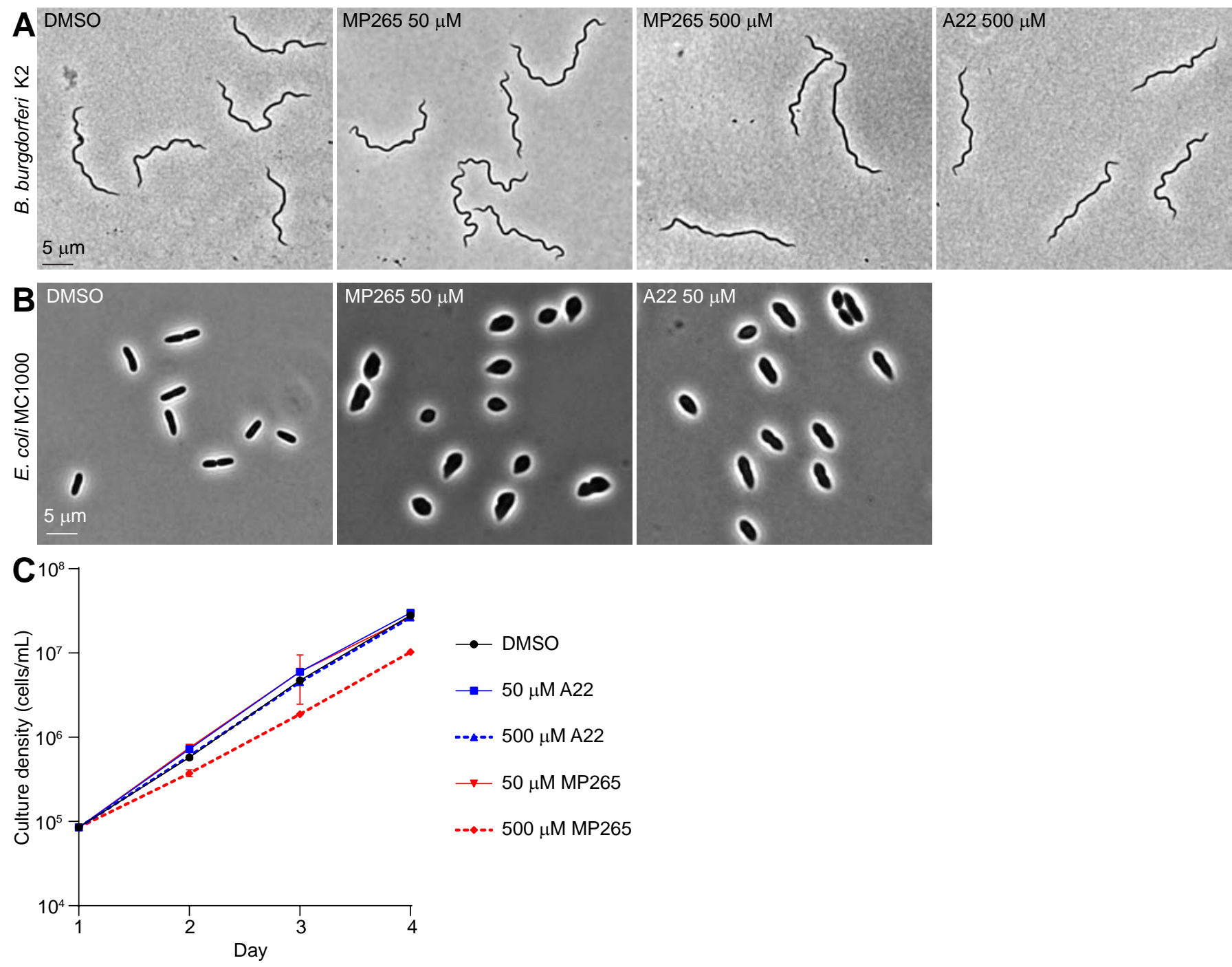

- DMSO

$\rightarrow 50 \mu \mathrm{M}$ A22

..... $500 \mu \mathrm{MA}$ A22

$\rightarrow 50 \mu \mathrm{M}$ MP265

..... $500 \mu \mathrm{M}$ MP265

Figure S7. Phenotypic characterization of $B$. burgdorferi following exposure to MreB inhibitors.

A. Phase contrast images of cells of $B$. burgdorferi strain K2 grown for two days in the presence of $0.1 \%$ DMSO or the indicated concentrations of MP265 or A22.

B. Phase contrast images of E. coli strain MC1000 grown for one hour in BSK-II medium supplemented with DMSO, MP265, or A22.

C. Growth curve of strain K2 treated with DMSO, MP265, or A22. Two replicate cultures were counted daily for each condition. Shown are means \pm standard deviations. 


\section{SUPPLEMENTAL MOVIE LEGENDS}

All movies were recorded by imaging live B. burgdorferi cells in BSK-H medium using darkfield microscopy. Stream acquisition with an exposure time of 200 ms was used. Shown are inverted images. Elapsed time (in seconds) is displayed in the top right corner. A 10- $\mu$ m scale bar is in the bottom right corner.

Movie_S1. A cell of strain CJW_Bb242 (control strain that carries a dcas9 shuttle vector but does not express a sgRNA) grown in the absence of IPTG.

Movie_S2. Cells of strain CJW_Bb242 grown in the presence of $0.1 \mathrm{mM}$ IPTG for two days.

Movie_S3. Cells of strain CJW_Bb313 (FlaB depletion strain carrying an all-in-one CRISPRi shuttle vector targeting $f l a B$ and the unmutated $\mathrm{P}_{p Q E 30}$ promoter controlling dcas 9 expression) grown in the absence of IPTG.

Movie_S4. A cell of strain CJW_Bb313 grown with $0.1 \mathrm{mM}$ IPTG for two days.

Movie_S5. Cell of strain CJW_Bb313 grown with $0.1 \mathrm{mM} \mathrm{IPTG} \mathrm{for} \mathrm{two} \mathrm{days.}$

Movie_S6. Cell of strain CJW_Bb313 grown with $0.1 \mathrm{mM} \mathrm{IPTG} \mathrm{for} \mathrm{two} \mathrm{days.}$

Movie_S7. Cells of strain CJW_Bb313 grown with $0.1 \mathrm{mM} \mathrm{IPTG} \mathrm{for} \mathrm{two} \mathrm{days.}$

Movie_S8. A cell of strain CJW_Bb313 grown with $0.1 \mathrm{mM}$ IPTG for two days. The cell appears almost perfectly straight, like cells of a $\Delta f l a B$ strain (12). The kink at the middle represents the division site. 


\section{SUPPLEMENTAL REFERENCES}

1. Gitai Z, Dye NA, Reisenauer A, Wachi M, Shapiro L. 2005. MreB actin-mediated segregation of a specific region of a bacterial chromosome. Cell 120:329-341.

2. Takacs CN, Poggio S, Charbon G, Pucheault M, Vollmer W, Jacobs-Wagner C. 2010. MreB drives de novo rod morphogenesis in Caulobacter crescentus via remodeling of the cell wall. J Bacteriol 192:1671-1684.

3. Iwai N, Nagai K, Wachi M. 2002. Novel S-benzylisothiourea compound that induces spherical cells in Escherichia coli probably by acting on a rod-shape-determining protein(s) other than penicillin-binding protein 2. Biosci Biotechnol Biochem 66:2658-2662.

4. Iwai N, Ebata T, Nagura H, Kitazume T, Nagai K, Wachi M. 2004. Structure-activity relationship of S-benzylisothiourea derivatives to induce spherical cells in Escherichia coli. Biosci Biotechnol Biochem 68:2265-2269.

5. Iwai N, Fujii T, Nagura H, Wachi M, Kitazume T. 2007. Structure-activity relationship study of the bacterial actin-like protein MreB inhibitors: effects of substitution of benzyl group in Sbenzylisothiourea. Biosci Biotechnol Biochem 71:246-248.

6. Slamti L, de Pedro MA, Guichet E, Picardeau M. 2011. Deciphering morphological determinants of the helix-shaped Leptospira. J Bacteriol 193:6266-6275.

7. Bono JL, Elias AF, Kupko JJ, 3rd, Stevenson B, Tilly K, Rosa P. 2000. Efficient targeted mutagenesis in Borrelia burgdorferi. J Bacteriol 182:2445-2452.

8. Adams PP, Flores Avile C, Popitsch N, Bilusic I, Schroeder R, Lybecker M, Jewett MW. 2017. In vivo expression technology and 5' end mapping of the Borrelia burgdorferi transcriptome identify novel RNAs expressed during mammalian infection. Nucleic Acids Res 45:775-792.

9. Arnold WK, Savage CR, Brissette CA, Seshu J, Livny J, Stevenson B. 2016. RNA-seq of Borrelia burgdorferi in multiple phases of growth reveals insights into the dynamics of gene expression, transcriptome architecture, and noncoding RNAs. PLoS One 11:e0164165. 
10. Takacs CN, Kloos ZA, Scott M, Rosa PA, Jacobs-Wagner C. 2018. Fluorescent proteins, promoters, and selectable markers for applications in the Lyme disease spirochete Borrelia burgdorferi. Appl Environ Microbiol 84:e01824-18.

11. Elias AF, Stewart PE, Grimm D, Caimano MJ, Eggers CH, Tilly K, Bono JL, Akins DR, Radolf JD, Schwan TG, Rosa P. 2002. Clonal polymorphism of Borrelia burgdorferi strain B31 MI: implications for mutagenesis in an infectious strain background. Infect Immun 70:2139-2150.

12. Motaleb MA, Corum L, Bono JL, Elias AF, Rosa P, Samuels DS, Charon NW. 2000. Borrelia burgdorferi periplasmic flagella have both skeletal and motility functions. Proc Natl Acad Sci U S A 97:10899-10904. 